\title{
Convergent Synthesis of Tetrasaccharide Fragment of Cervimycin K
}

\author{
Supporting Information
}

Jihun Kang and Young Ho Rhee*

Department of Chemistry, POSTECH, 77 Cheongam-Ro, Nam-Gu, Pohang, Kyungbuk, Republic of Korea, 37673

Table of Contents

1. General information

2. Synthesis of allene substrates 3

S2

3. Synthesis of alcohol substrates 4

4. Asymmetric addition of alcohol 4 to allenes 3 via palladium catalyst

S8

5. Synthesis of ene-allene 16

S13

6. Asymmetric addition of alcohol to allenes 16 via palladium catalyst S15 and ring-closing metathesis

7. Synthesis of 2,3,6-Trideoxy-tetrasaccharide moiety 1

8. Reference

9. 1H, 13C NMR and 2D NMR of new compounds

10. 1H NMR of Crude mixture: Determination of d.r for the addition reaction 


\section{General information}

Air and moisture sensitive reactions were carried out in oven-dried glassware sealed with rubber septa under a positive pressure of nitrogen. Similarly all solvents were dried and distilled according to the standard methods before use, then were transferred via syringe. Reactions were stirred using Teflon-coated magnetic stir bars. For all the reactions that require heating, oil bath was used as a heating source. $\operatorname{Pd}_{2}(\mathrm{dba})_{3}$, the Grubbs' catalysts were purchased from Aldrich Chemical, Strem Chemical Inc. Chiral Trost ligands were purchased from Strem Chemical Inc. and stored in glove box. Reactions were monitored by thin-layer chromatography on silicagel carried out on $0.25 \mathrm{~mm}$ E. Merck silica gel plates (60F- 254) using UV light as a visualizing agent and acidic p-anisaldehyde, and heat as developing agent. Flash chromatography on silicagel was carried out on Merck 60 silica gel (230-400 mesh). ${ }^{1} \mathrm{H}$ and ${ }^{13} \mathrm{C}$ NMR spectra were recorded on Bruker $(300 \mathrm{MHz}, 500 \mathrm{MHz}, 600 \mathrm{MHz})$ spectrometer. ${ }^{1} \mathrm{H}$ NMR spectra were referenced to $\mathrm{CDCl}_{3}(7.26 \mathrm{ppm})$, and reported as follows; chemical shift, multiplicity ( $\mathrm{s}=$ singlet, $\mathrm{d}$ $=$ doublet, $\mathrm{t}=$ triplet, $\mathrm{q}=$ quartet, $\mathrm{m}=$ multiplet $).$ Chemical shifts of the ${ }^{13} \mathrm{C}$ NMR spectra were measured relative to $\mathrm{CDCl}_{3}(77.23 \mathrm{ppm})$. Infrared spectra were recorded on a Shimadzu IR-470 spectrometer. Specific rotation data were measured on JASCO P-1020 Polarimeter. HPLC was performed with an Agilent Technologies 1220 infinity LC system. Melting points were measured on Electrothermal 9100. Mass spectral data were obtained from the Korea Basic Science Institute (Daegu) on a Jeol JMS 700 high resolution mass spectrometer (FAB) and Organic Chemistry Research Center in Sogang university on a Bruker Ultra High Resolution ESI Q-TOF MS / MS Compact System (ESI). 


\section{Synthesis of allene substrates 3}

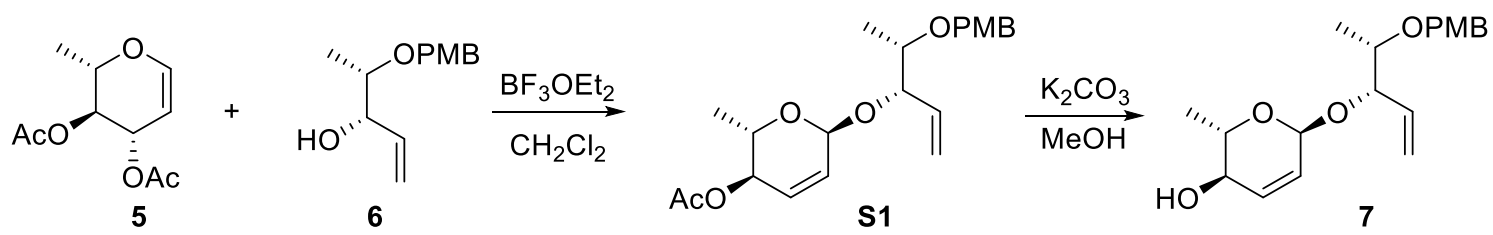

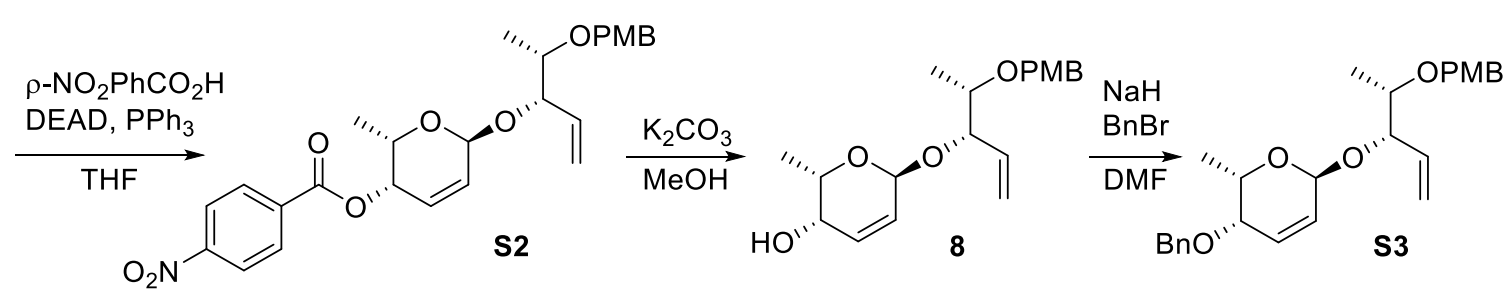

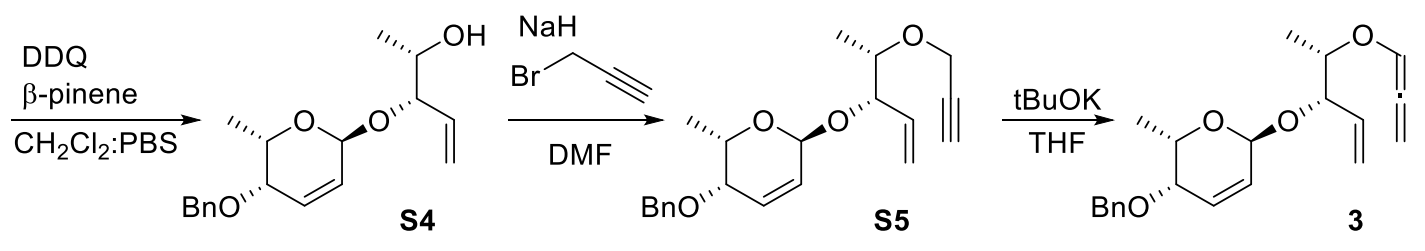<smiles>C=C[C@H](O[C@H]1C=C[C@@H](OC(C)=O)[C@@H](C)O1)[C@H](C)O[Mg]</smiles>

(2S,3R,6S)-6-(((3S,4S)-4-((4-methoxybenzyl)oxy)pent-1-en-3-yl)oxy)-2-methyl-3,6-dihydro-2H-pyran-3-yl acetate (S1): To a solution of $\mathbf{6}^{1}(2.40 \mathrm{~g}, 10.8 \mathrm{mmol})$ in $\mathrm{CH}_{2} \mathrm{Cl}_{2}(110 \mathrm{ml})$ was added commercially available 3,4di-O-acetyl-6-deoxy- $(L)$-glucal $5(2.78 \mathrm{~g}, 13.0 \mathrm{mmol})$ and $\mathrm{BF}_{3} \cdot \mathrm{OEt}_{2}(0.40 \mathrm{ml}, 3.2 \mathrm{mmol})$ at $-78^{\circ} \mathrm{C}$. The reaction mixture was then allowed to gradually warm to $-55^{\circ} \mathrm{C}$ over the course of $30 \mathrm{~min}$, and stirred for an additional $1 \mathrm{~h}$. The reaction mixture was quenched with pyridine $(0.39 \mathrm{ml}, 4.8 \mathrm{mmol})$ and saturated $\mathrm{NaHCO}_{3}(50 \mathrm{ml})$, and then the aqueous phase was extracted with $\mathrm{CH}_{2} \mathrm{Cl}_{2}(4 \times 150 \mathrm{ml})$. The organic phase was washed with brine, dried over $\mathrm{Na}_{2} \mathrm{SO}_{4}$ and concentrated under reduced pressure. The crude product was purified by flash column chromatography (Hexane:Ethyl acetate $=90: 10)$ to afford $\mathbf{S} 1$ as a colorless oil $(2.22 \mathrm{~g}, 5.9 \mathrm{mmol}, 55 \%$ yield). The diastereomer ratio (3.7:1) was determined by crude NMR.

$\mathrm{R}_{f} 0.34$ (Hexane:Ethyl acetate $\left.=80: 20\right) ;[\alpha]^{28} \mathrm{D}-109.59\left(c 0.84, \mathrm{CH}_{2} \mathrm{Cl}_{2}\right) ;{ }^{1} \mathrm{H}$ NMR $\left(500 \mathrm{MHz}, \mathrm{CDCl}_{3}\right): \delta=7.28-$ $7.26(\mathrm{~m}, 2 \mathrm{H}), 6.89-6.87(\mathrm{~m}, 2 \mathrm{H}), 5.92-5.83(\mathrm{~m}, 2 \mathrm{H}), 5.82-5.79(\mathrm{td}, J=1.82,10.36 \mathrm{~Hz}, 1 \mathrm{H}), 5.36-5.32(\mathrm{~m}, 2 \mathrm{H})$, 5.07-5.04 (td, $J=1.42,9.32 \mathrm{~Hz}, 1 \mathrm{H}), 4.59-4.51(\mathrm{q}, J=11.68 \mathrm{~Hz}, 2 \mathrm{H}), 4.13-4.10(\mathrm{t}, J=6.77 \mathrm{~Hz}, 1 \mathrm{H}), 4.01-3.95$ (m, $1 \mathrm{H}), 3.61(\mathrm{~s}, 3 \mathrm{H}), 3.58-3.53(\mathrm{qd}, J=6.40,6.41 \mathrm{~Hz}, 1 \mathrm{H}), 2.09$ (s, 3H), $1.17-1.15(\mathrm{~d}, J=6.25 \mathrm{~Hz}, 3 \mathrm{H}), 1.13-$ $1.11(\mathrm{~d}, J=6.41,3 \mathrm{H}) ;{ }^{13} \mathrm{C} \mathrm{NMR}\left(125 \mathrm{MHz}, \mathrm{CDCl}_{3}\right): \delta=170.7,159.3,136.4,131.1,129.8,129.2,128.2,117.0$, 114.0, 95.3, 82.8, 71.5, 71.2, 65.0, 55.5, 21.2, 17.8, 16.2; IR (NaCl): v 3075, 3052, 2979, 2934, 2903, 2837, 1741, 1613, 1586, 1514, 1465, $1447 \mathrm{~cm}^{-1}$; HRMS (ESI) m/z: [M+Na] $]^{+}$Calcd for $\mathrm{C}_{21} \mathrm{H}_{28} \mathrm{NaO}_{6} 399.1778$; Found 399.1781. 
<smiles>C=C[C@@H](O[C@@H]1C=C[C@@H](O)[C@@H](C)O1)[C@@H](C)O[Mg]</smiles>

$(2 S, 3 R, 6 S)-6-(((3 S, 4 S)-4-((4-m e t h o x y b e n z y l) o x y)$ pent-1-en-3-yl)oxy)-2-methyl-3,6-dihydro-2H-pyran-3-ol (7): A solution of $\mathbf{S 1}(2.032 \mathrm{~g}, 5.4 \mathrm{mmol})$ and $\mathrm{K}_{2} \mathrm{CO}_{3}(0.52 \mathrm{~g}, 3.8 \mathrm{mmol})$ in $\mathrm{MeOH}(54 \mathrm{ml})$ was stirred for $30 \mathrm{~min}$ at room temperature. The crude mixture was filtered with a pad of celite (rinse; Ethyl acetate $150 \mathrm{ml}$ ), and then concentrated under reduced pressure. The crude product was purified by flash column chromatography (Hexane:Ethyl acetate $=80: 20)$ to afford 7 as a colorless oil $(1.61 \mathrm{~g}, 4.8 \mathrm{mmol}, 89 \%)$.

$\mathrm{R}_{f} 0.44$ (Hexane:Ethyl acetate $\left.=60: 40\right) ;[\alpha]^{28} \mathrm{D}-54.69\left(c 2.20, \mathrm{CH}_{2} \mathrm{Cl}_{2}\right) ;{ }^{1} \mathrm{H}$ NMR $\left(500 \mathrm{MHz}, \mathrm{CDCl}_{3}\right): \delta=7.28-$ $7.26(\mathrm{~m}, 2 \mathrm{H}), 6.89-6.87(\mathrm{~m}, 2 \mathrm{H}), 5.91-5.84(\mathrm{~m}, 2 \mathrm{H}), 5.76-5.72(\mathrm{td}, J=2.38,10.14 \mathrm{~Hz}, 1 \mathrm{H}), 5.36-5.32(\mathrm{~m}, 1 \mathrm{H})$, 5.21-5.19 (m, 1H), $5.16(\mathrm{~s}, 1 \mathrm{H}), 4.58-4.51(\mathrm{~m}, 2 \mathrm{H}), 4.13-4.10(\mathrm{t}, J=7.14 \mathrm{~Hz}, 1 \mathrm{H}), 3.84-3.80(\mathrm{~m}, 4 \mathrm{H}), 3.75-3.69$ $(\mathrm{m}, 1 \mathrm{H}), 3.57-3.52(\mathrm{qd}, J=6.31,6.31 \mathrm{~Hz}, 1 \mathrm{H}), 1.93(\mathrm{~s}, 1 \mathrm{H}), 1.26-1.24(\mathrm{~d}, J=6.31 \mathrm{~Hz}, 3 \mathrm{H}), 1.12-1.11(\mathrm{~d}, J=6.37$ $\mathrm{Hz}, 3 \mathrm{H}) ;{ }^{13} \mathrm{C}$ NMR $\left(125 \mathrm{MHz}, \mathrm{CDCl}_{3}\right): \delta=159.3,136.4,133.6,131.1,129.2,126.9,117.0,113.9,95.2,82.7$, 77.2, 71.5, 69.9, 68.1, 55.4, 17.7, 16.2; IR (NaCl): v 3433, 3077, 3041, 2975, 2933, 2899, 1643, 1612, 1586, 1513 , $1456,1400 \mathrm{~cm}^{-1}$; HRMS (ESI) m/z: [M+Na] $]^{+}$Calcd for $\mathrm{C}_{19} \mathrm{H}_{26} \mathrm{NaO}_{5}$ 357.1672; Found 357.1676.<smiles>C=C[C@H](O[C@H]1C=C[C@@H](OC(=O)c2ccc([N+](=O)[O-])cc2)[C@@H](C)O1)[C@@H](C)OCCC</smiles>

(2S,3S,6S)-6-(((3S,4S)-4-((4-methoxybenzyl)oxy)pent-1-en-3-yl)oxy)-2-methyl-3,6-dihydro-2H-pyran-3-yl4-nitrobenzoate (S2): To a solution of $7(2.20 \mathrm{~g}, 6.6 \mathrm{~mol})$ in anhydrous THF $(17.3 \mathrm{ml})$, diethyl azidocarboxylate (6.6 ml, $13.2 \mathrm{~mol}, 2 \mathrm{M}$ in THF) and triphenyl phosphine ( $3.54 \mathrm{~g}, 13.5 \mathrm{~mol}$ ) were suspended at room temperature. The solution was cooled to $0^{\circ} \mathrm{C}$. After $10 \mathrm{~min}$, p-nitrobenzoic acid $(2.21 \mathrm{~g}, 13.2 \mathrm{~mol})$ was added slowly to reaction mixture at $0{ }^{\circ} \mathrm{C}$. The resulting mixture was stirred for $1 \mathrm{~h}$ at room temperature. The reaction mixture was quenched with saturated $\mathrm{NaHCO}_{3}(50 \mathrm{ml})$, and then the aqueous phase was extracted with $\mathrm{Et}_{2} \mathrm{O}(4 \times 100 \mathrm{ml})$. The organic phase was washed with brine, dried over $\mathrm{Na}_{2} \mathrm{SO}_{4}$ and concentrated under reduced pressure. the crude product was purified by flash column chromatography (Hexane:Ethyl acetate $=90: 10)$ to afford $\mathbf{S} 2$ as a white solid $(2.66 \mathrm{~g}$, $5.5 \mathrm{mmol}, 83 \%)$.

m.p. 93-96 ${ }^{\circ} \mathrm{C}$; $\mathrm{R}_{f} 0.57$ (Hexane:Ethyl acetate $\left.=80: 20\right) ;[\alpha]^{28} \mathrm{D}+118.61\left(c 1.21, \mathrm{CH}_{2} \mathrm{Cl}_{2}\right) ;{ }^{1} \mathrm{H}$ NMR $(500 \mathrm{MHz}$, $\left.\mathrm{CDCl}_{3}\right): \delta=8.28-8.22(\mathrm{~m}, 4 \mathrm{H}), 7.28-7.26(\mathrm{~m}, 2 \mathrm{H}), 6.88-6.86(\mathrm{~m}, 2 \mathrm{H}), 6.19-6.16(\mathrm{dd}, J=5.39,9.84 \mathrm{~Hz}, 1 \mathrm{H}), 6.09-$ 6.07 (dd, $J=3.09,9.87 \mathrm{~Hz}, 1 \mathrm{H}), 5.91-5.84(\mathrm{ddd}, J=7.29,10.04,17.44 \mathrm{~Hz}, 1 \mathrm{H}), 5.36-5.30(\mathrm{~m}, 2 \mathrm{H}), 5.23-5.21$ (d, $J=10.51 \mathrm{~Hz}, 1 \mathrm{H}), 5.1-5.14(\mathrm{dd}, J=2.15,5.39 \mathrm{~Hz}, 1 \mathrm{H}), 4.59-4.50(\mathrm{dd}, J=11.45,34.76 \mathrm{~Hz}, 2 \mathrm{H}), 4.36-4.32(\mathrm{qd}, J$ $=2.14,6.46 \mathrm{~Hz}, 1 \mathrm{H}), 4.16-4.12(\mathrm{dd}, J=6.49,6.49 \mathrm{~Hz}, 1 \mathrm{H}), 3.80(\mathrm{~s}, 1 \mathrm{H}), 3.59-3.54(\mathrm{qd}, J=6.49,6.49 \mathrm{~Hz}, 1 \mathrm{H})$, $1.24-1.22$ (d, $J=6.49 \mathrm{~Hz}, 3 \mathrm{H}), 1.14-1.12(\mathrm{~d}, J=6.22 \mathrm{~Hz}, 3 \mathrm{H}) ;{ }^{13} \mathrm{C} \mathrm{NMR}\left(125 \mathrm{MHz}, \mathrm{CDCl}_{3}\right): \delta=164.5,159.3$, $150.9,136.3,135.5,131.6,131.1,129.3,125.4,123.7,117.3,114.0,95.2,82.9,77.3,91.6,67.1,64.8,55.5,16.3$, 16.0; IR (NaCl): v 3110, 3078, 3054, 2981, 2937, 2899, 2837, 1722, 1610, 1586, 1528, $1460 \mathrm{~cm}^{-1}$; HRMS (ESI) $\mathrm{m} / \mathrm{z}$ : $[\mathrm{M}+\mathrm{Na}]^{+}$Calcd for $\mathrm{C}_{26} \mathrm{H}_{29} \mathrm{NNaO}_{8} 506.1785$; Found 506.1788 . 
<smiles>[R16]O[C@H](C)[C@@H](C=C)O[C@@H]1C=C[C@@H](O)[C@@H](C)O1</smiles>

(2S,3S,6S)-6-(((3S,4S)-4-((4-methoxybenzyl)oxy)pent-1-en-3-yl)oxy)-2-methyl-3,6-dihydro-2H-pyran-3-ol (8): A solution of $\mathbf{S 2}(2.66 \mathrm{~g}, 5.5 \mathrm{mmol})$ and $\mathrm{K}_{2} \mathrm{CO}_{3}(0.53 \mathrm{~g}, 3.9 \mathrm{mmol})$ in $\mathrm{MeOH}(55 \mathrm{ml})$ was stirred for $30 \mathrm{~min}$ at room temperature. The crude mixture was filtered with a pad of celite (rinse; Ethyl acetate $150 \mathrm{ml}$ ), and then concentrated under reduced pressure. The crude product was purified by flash column chromatography (Hexane:Ethyl acetate $=80: 20$ ) to afford $\mathbf{8}$ as a colorless oil $(1.67 \mathrm{~g}, 5.0 \mathrm{mmol}, 91 \%)$.

$\mathrm{R}_{f} 0.43$ (Hexane:Ethyl acetate $\left.=60: 40\right) ;[\alpha]^{28}{ }_{\mathrm{D}}+50.70\left(c 1.24, \mathrm{CH}_{2} \mathrm{Cl}_{2}\right) ;{ }^{1} \mathrm{H}$ NMR $\left(500 \mathrm{MHz}, \mathrm{CDCl}_{3}\right): \delta=7.27-$ $7.25(\mathrm{~m}, 2 \mathrm{H}), 6.88-6.86(\mathrm{~m}, 2 \mathrm{H}), 6.18-6.15(\mathrm{dd}, J=5.54,9.95 \mathrm{~Hz}, 1 \mathrm{H}), 5.89-5.82(\mathrm{~m}, 2 \mathrm{H}), 5.34-5.30(\mathrm{td}, J=1.31$, $17.21 \mathrm{~Hz}, 1 \mathrm{H}), 5.21-5.19(\mathrm{~d}, J=10.60 \mathrm{~Hz}, 1 \mathrm{H}), 5.18-5.17(\mathrm{~d}, J=2.97 \mathrm{~Hz}, 1 \mathrm{H}), 4.58-4.50$ (dd, $J=11.44,26.98$ $\mathrm{Hz}, 2 \mathrm{H}), 4.13-4.08(\mathrm{~m}, 2 \mathrm{H}), 3.80(\mathrm{~s}, 1 \mathrm{H}), 3.59-3.52(\mathrm{~m}, 2 \mathrm{H}), 1.63-1.61(\mathrm{~d}, J=10.42 \mathrm{~Hz}, 1 \mathrm{H}), 1.23-1.22(\mathrm{~d}, J=$ $6.64 \mathrm{~Hz}, 3 \mathrm{H}), 1.12-1.11(\mathrm{~d}, J=6.37 \mathrm{~Hz}, 3 \mathrm{H}) ;{ }^{13} \mathrm{C}$ NMR $\left(125 \mathrm{MHz}, \mathrm{CDCl}_{3}\right): \delta=159.3,136.4,131.1,130.5,129.2$, 128.5, 117.1, 113.9, 95.3, 82.4 , 77.3, 71.5, 66.5, 64.3, 55.5, 16.3, 15.9; IR (NaCl): v 3441, 3078, 3040, 2979, 2934 , 2903, 2837, 1613, 1513, 1458, 1403, $1377 \mathrm{~cm}^{-1}$; HRMS (ESI) m/z: $[\mathrm{M}+\mathrm{Na}]^{+}$Calcd for $\mathrm{C}_{19} \mathrm{H}_{26} \mathrm{NaO}_{5} 357.1672$; Found 357.1674.<smiles>[B]O[C@@H]1C=C[C@@H](O[C@H](C=C)[C@@H](C)O[Mg])O[C@@H]1C</smiles>

(2S,3S,6S)-3-(benzyloxy)-6-(((3S,4S)-4-((4-methoxybenzyl)oxy)pent-1-en-3-yl)oxy)-2-methyl-3,6-dihydro-2 H-pyran (S3): To a suspension of $\mathrm{NaH}(0.600 \mathrm{~g}, 15.0 \mathrm{mmol}, 60 \%$ dispersion in mineral oil $)$ in DMF was added $8(1.672 \mathrm{~g}, 5.0 \mathrm{mmol})$ in DMF $(25 \mathrm{ml})$ at $0{ }^{\circ} \mathrm{C}$. The reaction mixture was stirred for $10 \mathrm{~min}$ at room temperature. Benzyl bromide $(1.2 \mathrm{ml}, 10.0 \mathrm{mmol})$ was added to a reaction mixture at $0{ }^{\circ} \mathrm{C}$. The resulting mixture was stirred for $1.5 \mathrm{~h}$ at room temperature. The reaction mixture was quenched with water $(50 \mathrm{ml})$, and then the aqueous phase was extracted with $\mathrm{Et}_{2} \mathrm{O}(4 \times 100 \mathrm{ml})$. The organic phase was washed with brine, dried over $\mathrm{Na}_{2} \mathrm{SO}_{4}$ and concentrated under reduced pressure. The crude product was purified by flash column chromatography (Hexane:Ethyl acetate $=90: 10$ ) to afford $\mathbf{S 3}$ as a colorless oil $(1.897 \mathrm{~g}, 4.5 \mathrm{mmol}, 90 \%)$.

$\mathrm{R}_{f} 0.59$ (Hexane:Ethyl acetate $\left.=80: 20\right) ;[\alpha]^{29}{ }_{\mathrm{D}}+90.99\left(c 1.60, \mathrm{CH}_{2} \mathrm{Cl}_{2}\right) ;{ }^{1} \mathrm{H} \mathrm{NMR}\left(500 \mathrm{MHz}, \mathrm{CDCl}_{3}\right): \delta=77.36-$ $7.26(\mathrm{~m}, 7 \mathrm{H}), 6.89-6.86(\mathrm{~m}, 2 \mathrm{H}), 6.15-6.12(\mathrm{dd}, J=5.15,10.05 \mathrm{~Hz}, 1 \mathrm{H}), 6.01-5.98(\mathrm{dd}, J=3.03,10.05 \mathrm{~Hz}, 1 \mathrm{H})$, 5.89- $5.82(\mathrm{ddd}, J=6.91,10.58,17.31 \mathrm{~Hz}, 1 \mathrm{H}), 5.34-5.30(\mathrm{td}, J=1.21,17.19 \mathrm{~Hz}, 1 \mathrm{H}), 5.26-5.25(\mathrm{~d}, J=2.84 \mathrm{~Hz}$, $1 \mathrm{H}), 5.20-5.18(\mathrm{~d}, J=10.58 \mathrm{~Hz}, 1 \mathrm{H}), 4.66-4.51(\mathrm{~m}, 4 \mathrm{H}), 4.17-4.11(\mathrm{~m}, 2 \mathrm{H}), 3.80(\mathrm{~s}, 3 \mathrm{H}), 3.57-3.51(\mathrm{~m}, 2 \mathrm{H})$, $1.29-1.28(\mathrm{~d}, J=6.66 \mathrm{~Hz}, 3 \mathrm{H}), 1.12-1 . .11(\mathrm{~d}, J=6.47 \mathrm{~Hz}, 3 \mathrm{H}) ;{ }^{13} \mathrm{C}$ NMR $\left(125 \mathrm{MHz}, \mathrm{CDCl}_{3}\right): \delta=159.3,138.9$, $136.4,131.1,129.9,129.2,128.5,127.9,127.7,127.6,117.0,113.9,95.3,81.9,77.1,71.5,70.8,69.7,66.6,55.5$, 16.2, 16.1; IR (NaCl): v 3066, 3034, 2978, 2933, 2897, 2870, 2836, 1613, 1586, 1513, 1455, $1402 \mathrm{~cm}^{-1}$; HRMS (ESI) m/z: $[\mathrm{M}+\mathrm{Na}]^{+} \mathrm{Calcd}$ for $\mathrm{C}_{26} \mathrm{H}_{32} \mathrm{NaO}_{5}$ 447.2142; Found 447.2146. 
<smiles>C=C[C@@H](O[C@@H]1C=C[C@@H](O)[C@@H](C)O1)[C@@H](C)O</smiles>

(2S,3S)-3-(((2S,5S,6S)-5-(benzyloxy)-6-methyl-5,6-dihydro-2H-pyran-2-yl)oxy)pent-4-en-2-ol (S4): To a solution of $\mathbf{S 3}(1.868 \mathrm{~g}, 4.4 \mathrm{mmol})$ in a mixed solvent $\left(\mathrm{CH}_{2} \mathrm{Cl}_{2} / \mathrm{pH} 7.0\right.$ phosphate buffer, v/v 20/1, $\left.440 \mathrm{ml}\right)$ at $0{ }^{\circ} \mathrm{C}$ was added DDQ $(1.998 \mathrm{~g}, 8.8 \mathrm{mmol})$. After stirring at $0{ }^{\circ} \mathrm{C}$ for $5 \mathrm{~h}$, The reaction mixture was quenched with saturated $\mathrm{NaHCO}_{3}(50 \mathrm{ml})$, and then the aqueous phase was extracted with $\mathrm{CH}_{2} \mathrm{Cl}_{2}(4 \times 100 \mathrm{ml})$. The organic phase was washed with brine, dried over $\mathrm{Na}_{2} \mathrm{SO}_{4}$ and concentrated under reduced pressure. The crude product was purified by flash column chromatography (Hexane:Ethyl acetate $=80: 20$ ) to afford $\mathbf{S} \mathbf{4}$ as a colorless oil (1.156 $\mathrm{g}, 3.8 \mathrm{mmol}, 86 \%$ yield).

$\mathrm{R}_{f} 0.55$ (Hexane:Ethyl acetate $\left.=60: 40\right) ;[\alpha]^{29}{ }_{\mathrm{D}}+133.80\left(c 0.58, \mathrm{CH}_{2} \mathrm{Cl}_{2}\right) ;{ }^{1} \mathrm{H} \mathrm{NMR}\left(500 \mathrm{MHz}, \mathrm{CDCl}_{3}\right): \delta=7.33-$ $7.25(\mathrm{~m}, 5 \mathrm{H}), 6.17-6.14(\mathrm{dd}, J=5.15,10.08 \mathrm{~Hz}, 1 \mathrm{H}), 6.03-6.01(\mathrm{dd}, J=3.01,10.13 \mathrm{~Hz}, 1 \mathrm{H}), 5.85-5.78$ (ddd, $J=$ $7.67,10.56,17.79 \mathrm{~Hz}, 1 \mathrm{H}), 5.33-5.30$ (td, $J=1.28,17.4 \mathrm{~Hz}), 5.24-5.22$ (d, $J=10.48 \mathrm{~Hz}, 1 \mathrm{H}), 5.20-5.19$ (d, $J=$ $2.86 \mathrm{~Hz}, 1 \mathrm{H}), 4.65-4.54(\mathrm{dd}, J=11.89,40.87 \mathrm{~Hz}, 2 \mathrm{H}), 4.13-4.08(\mathrm{qd}, J=2.76,6.75 \mathrm{~Hz}, 1 \mathrm{H}), 3.83-3.80(\mathrm{dd}, J=$ 7.46, $7.46 \mathrm{~Hz}, 1 \mathrm{H}), 3.71-3.65(\mathrm{~m}, 1 \mathrm{H}), 3.54-3.52(\mathrm{dd}, J=2.62,5.18 \mathrm{~Hz}, 1 \mathrm{H}), 2.46-2.45(\mathrm{~d}, J=3.06 \mathrm{~Hz}, 1 \mathrm{H})$, $1.28-1.27(\mathrm{~d}, 6.73 \mathrm{~Hz}, 1 \mathrm{H}), 1.16-1.14(\mathrm{~d}, J=6.47 \mathrm{~Hz}, 1 \mathrm{H}) ;{ }^{13} \mathrm{C} \mathrm{NMR}\left(125 \mathrm{MHz}, \mathrm{CDCl}_{3}\right): \delta=138.8,136.7,129.6$, 128.6, 128.1, 127.9, 127.8, 118.1, 95.7, 85.6, 70.9, 69.8, 69.5, 66.9, 18.6, 16.0; IR (NaCl): v 3428, 3089, 3064, 3034, 2977, 2914, 2876, 1454, 1401, 1343, 1307, $1193 \mathrm{~cm}^{-1}$; HRMS (ESI) m/z: [M+Na] $]^{+}$Calcd for $\mathrm{C}_{18} \mathrm{H}_{24} \mathrm{NaO}_{4}$ 327.1567; Found 327.1569.<smiles>C#CCO[C@H](C)[C@@H](C=C)O[C@@H]1C=C[C@@H](O)[C@H](C)O1</smiles>

(2S,3S,6S)-3-(benzyloxy)-2-methyl-6-(((3S,4S)-4-(prop-2-yn-1-yloxy)pent-1-en-3-yl)oxy)-3,6-dihydro-2H-p yran (S5): To a suspension of $\mathrm{NaH}(0.432 \mathrm{~g}, 10.8 \mathrm{mmol}, 60 \%$ dispersion in mineral oil) in DMF was added $\mathbf{S} 4$ $(1.096 \mathrm{~g}, 3.6 \mathrm{mmol})$ in DMF $(20 \mathrm{ml})$ at $0{ }^{\circ} \mathrm{C}$ under nitrogen atmosphere. The reaction mixture was stirred for 10 min at room temperature. The solution of propargyl bromide $(1.7 \mathrm{ml}, 14.4 \mathrm{mmol}, 80 \% \mathrm{wt} \%$ in Toluene) was added to a reaction mixture at $0{ }^{\circ} \mathrm{C}$. The resulting mixture was stirred for $8 \mathrm{~h}$ at room temperature. The reaction mixture was quenched with water $(50 \mathrm{ml})$, and then the aqueous phase was extracted with $\mathrm{Et}_{2} \mathrm{O}(4 \times 100 \mathrm{ml})$. The organic phase was washed with brine, dried over $\mathrm{Na}_{2} \mathrm{SO}_{4}$ and concentrated under reduced pressure. The crude product was purified by flash column chromatography (Hexane:Ethyl acetate $=90: 10)$ to afford $\mathbf{S 5}$ as a colorless oil (1.12 g, $3.3 \mathrm{mmol}, 92 \%)$.

$\mathrm{R}_{f} 0.51$ (Hexane:Ethyl acetate $\left.=80: 20\right) ;[\alpha]^{29} \mathrm{D}+101.66\left(c 2.04, \mathrm{CH}_{2} \mathrm{Cl}_{2}\right) ;{ }^{1} \mathrm{H} \mathrm{NMR}\left(500 \mathrm{MHz}, \mathrm{CDCl}_{3}\right): \delta=7.35-$ $7.26(\mathrm{~m}, 5 \mathrm{H}), 6.15-6.12(\mathrm{dd}, J=5.24,10.21 \mathrm{~Hz}, 1 \mathrm{H}), 6.07-6.04(\mathrm{dd}, J=3.07,10.21 \mathrm{~Hz}, 1 \mathrm{H}), 5.87-5.80(\mathrm{ddd}, J=$ 7..06, 10.53, $17.49 \mathrm{~Hz}, 1 \mathrm{H}), 5.33-5.30$ (d, $J=17.49 \mathrm{~Hz}, 1 \mathrm{H}), 5.26-5.25$ (d, $J=3.07 \mathrm{~Hz}, 1 \mathrm{H}), 5.20-5.18$ (d, $J=$ $10.53 \mathrm{~Hz}, 1 \mathrm{H}), 4.65-4.54$ (dd, $J=11.92,41.71 \mathrm{~Hz}, 2 \mathrm{H}), 4.28-4.20$ (m, 2H), 4.14-4.09 (m, 2H), 3.68-3.63 (qd, $J=$ $6.43,6.43 \mathrm{~Hz}, 1 \mathrm{H}$ ), $3.52-3.51$ (dd, $J=2.73,5.24 \mathrm{~Hz}, 1 \mathrm{H}), 2.40-2.39$ (t, $J=2.33 \mathrm{~Hz}, 1 \mathrm{H}), 1.28-1.27$ (d, $J=6.75$ $\mathrm{Hz}, 3 \mathrm{H}), 1.13-1.12(\mathrm{~d}, J=6.43 \mathrm{~Hz}, 3 \mathrm{H}) ;{ }^{13} \mathrm{C}$ NMR $\left(125 \mathrm{MHz}, \mathrm{CDCl}_{3}\right): \delta=138.9,136.2,129.8,128.5,127.9$, 127.7, 127.6, 117.2, 95.4, 82.1, 80.4, 74.0, 70.8, 69.6, 66.6, 57.3, 16.1, 16.1; IR (NaCl): v 3293, 3086, 3066, 3033, 2980, 2934, 2896, 1497, 1453, 1401, 1379, $1344 \mathrm{~cm}^{-1}$; HRMS (ESI) m/z: $[\mathrm{M}+\mathrm{Na}]^{+}$Calcd for $\mathrm{C}_{21} \mathrm{H}_{26} \mathrm{NaO}_{4}$ 365.1723; Found 365.1722. 
<smiles>C=C[C@H](OC=[IH])[C@@H](C)O[C@H]1C=C[C@@H](O)[C@H](C)O1</smiles>

(2S,3S,6S)-3-(benzyloxy)-2-methyl-6-(((3S,4S)-4-(propa-1,2-dien-1-yloxy)pent-1-en-3-yl)oxy)-3,6-dihydro2H-pyran (3): To a solution of $\mathbf{S 5}(1.061 \mathrm{~g}, 3.09 \mathrm{mmol})$ in THF $(10.3 \mathrm{ml})$, was added tBuOK $(0.173 \mathrm{~g}, 1.55$ mmol) at $0{ }^{\circ} \mathrm{C}$ and stirred for $2.5 \mathrm{~h}$ at $0{ }^{\circ} \mathrm{C}$. The crude mixture was filtered with a pad of celite (rinse; $\mathrm{Et}_{2} \mathrm{O} 150$ $\mathrm{ml}$ ), and then concentrated under reduced pressure. The crude product was purified by flash column chromatography (Hexane:Ethyl acetate $=80: 20)$ to afford 3 as a colorless oil $(0.918 \mathrm{~g}, 2.68 \mathrm{mmol}, 87 \%)$.

$\mathrm{R}_{f} 0.45$ (Hexane:Ethyl acetate $\left.=90: 10\right) ;[\alpha]^{29} \mathrm{D}+76.73\left(c 1.71, \mathrm{CH}_{2} \mathrm{Cl}_{2}\right) ;{ }^{1} \mathrm{H}$ NMR $\left(500 \mathrm{MHz}, \mathrm{CDCl}_{3}\right): \delta=7.35-$ $7.26(\mathrm{~m}, 5 \mathrm{H}), 6.69-6.66(\mathrm{t}, J=5.99 \mathrm{~Hz}, 1 \mathrm{H}), 6.15-6.12(\mathrm{dd}, J=5.19,10.08 \mathrm{~Hz}, 1 \mathrm{H}), 6.03-6.01(\mathrm{dd}, J=3.05,10.08$ $\mathrm{Hz}, 1 \mathrm{H}), 5.88-5.81$ (ddd, $J=6.87,10.57,17.44 \mathrm{~Hz}, 1 \mathrm{H}), 5.44-5.39(\mathrm{~m}, 2 \mathrm{H}), 5.35-5.31(\mathrm{td}, J=1.46,17.44 \mathrm{~Hz}$, $1 \mathrm{H}), 5.22-5.20(\mathrm{~m}, 2 \mathrm{H}), 4.66-4.55(\mathrm{dd}, J=12.04,41.34 \mathrm{~Hz}, 2 \mathrm{H}), 4.23-4.20(\mathrm{dd}, J=6.32,6.32 \mathrm{~Hz}, 1 \mathrm{H}), 4.14-4.09$ (qd, $J=2.65,6.67 \mathrm{~Hz}, 1 \mathrm{H}), 3.88-3.83(\mathrm{qd}, J=6.37,6.37 \mathrm{~Hz}, 1 \mathrm{H}), 3.52-3.50(\mathrm{dd}, J=2.70,5.23 \mathrm{~Hz}, 1 \mathrm{H}), 1.29-$ $1.28(\mathrm{~d}, J=6.61,1 \mathrm{H}), 1.16-1.15(\mathrm{~d}, J=6.37 \mathrm{~Hz}, 1 \mathrm{H}) ;{ }^{13} \mathrm{C} \mathrm{NMR}\left(125 \mathrm{MHz}, \mathrm{CDCl}_{3}\right): \delta=138.8,135.7,129.7$, $128.5,127.7,127.6,121.1,117.3,95.2,90.6,80.6,76.7,70.8,69.6,66.7,16.1,15.8 ; \mathrm{IR}(\mathrm{NaCl}): v$ 3085, 3065, 3035, 2980, 2934, 2897, 1952, 1497, 1447, 1401, 1378, $1347 \mathrm{~cm}^{-1}$; HRMS (ESI) m/z: $[\mathrm{M}+\mathrm{Na}]^{+}$Calcd for $\mathrm{C}_{21} \mathrm{H}_{26} \mathrm{NaO}_{4}$ 365.1723; Found 365.1727.

\section{Synthesis of alcohol substrates 4}

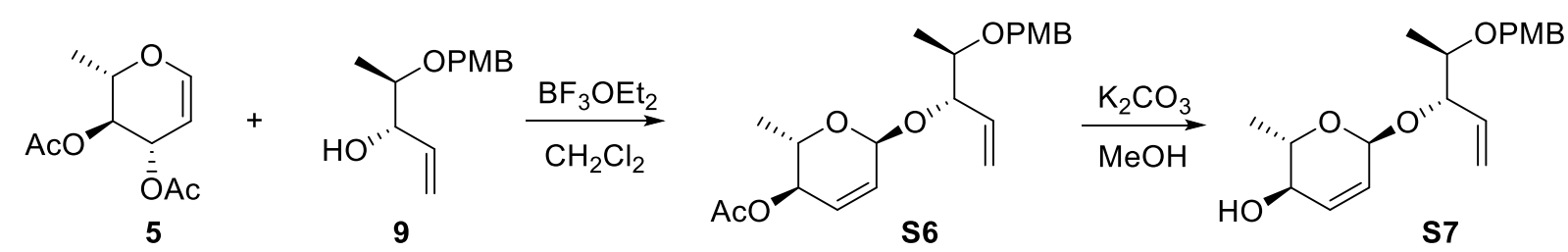<smiles>C=C[C@H](O[C@H]1C=C[C@@H](O)[C@@H](C)O1)[C@H](C)O[C@H]1C=C[C@@H](O)[C@@H](C)O1</smiles><smiles>C=C[C@H](O[C@H]1C=C[C@@H](O[14CH3])[C@@H](C)O1)C(C)O[Mg]</smiles>

(2S,3R,6S)-6-(((3S,4R)-4-((4-methoxybenzyl)oxy)pent-1-en-3-yl)oxy)-2-methyl-3,6-dihydro-2H-pyran-3-yl acetate (S6): To a solution of $\mathbf{9}^{1}(2.54 \mathrm{~g}, 11.4 \mathrm{mmol})$ in anhydrous $\mathrm{CH}_{2} \mathrm{Cl}_{2}(110 \mathrm{ml})$ was added commercially available 3,4-di-O-acetyl-6-deoxy-( $L$-glucal $5(2.91 \mathrm{~g}, 13.6 \mathrm{mmol})$ and $\mathrm{BF}_{3} \cdot \mathrm{OEt}_{2}(0.42 \mathrm{ml}, 3.4 \mathrm{mmol})$ at $-78{ }^{\circ} \mathrm{C}$. The reaction mixture was then allowed to gradually warm to $-50^{\circ} \mathrm{C}$ over the course of $30 \mathrm{~min}$, and stirred for an 
additional $1 \mathrm{~h}$. The reaction mixture was quenched with pyridine $(0.41 \mathrm{ml}, 5.1 \mathrm{mmol})$ and saturated $\mathrm{NaHCO}_{3}(50$ $\mathrm{ml})$, and then the aqueous phase was extracted with $\mathrm{CH}_{2} \mathrm{Cl}_{2}(4 \times 150 \mathrm{ml})$. The organic phase was washed with brine, dried over $\mathrm{Na}_{2} \mathrm{SO}_{4}$ and concentrated under reduced pressure. The crude product was purified by flash column chromatography (Hexane:Ethyl acetate $=90: 10)$ to afford $\mathbf{S 6}$ as a colorless oil $(2.45 \mathrm{~g}, 6.5 \mathrm{mmol}, 57 \%$ yield). The diastereomer ratio (4.0:1) was determined by crude NMR.

$\mathrm{R}_{f} 0.33$ (Hexane:Ethyl acetate $\left.=80: 20\right) ;[\alpha]^{27} \mathrm{D}-101.15\left(c 0.67, \mathrm{CH}_{2} \mathrm{Cl}_{2}\right) ;{ }^{1} \mathrm{H}$ NMR $\left(500 \mathrm{MHz}, \mathrm{CDCl}_{3}\right): \delta=7.28-$ $7.26(\mathrm{~m}, 2 \mathrm{H}), 6.89-6.87(\mathrm{~m}, 2 \mathrm{H}), 5.93-5.86(\mathrm{ddd}, J=6.27,10.68,17.09 \mathrm{~Hz}, 2 \mathrm{H}), 5.85$ (s, 2H), 5.34-5.30 (td, $J=$ $1.55,17.25 \mathrm{~Hz}, 1 \mathrm{H}), 4.53(\mathrm{~s}, 2 \mathrm{H}), 4.29-4.27(\mathrm{~m}, 1 \mathrm{H}), 4.01-3.98(\mathrm{~m}, 1 \mathrm{H}), 3.81(\mathrm{~s}, 3 \mathrm{H}), 3.59-3.56(\mathrm{~m}, 1 \mathrm{H}), 2.09(\mathrm{~s}$, $3 \mathrm{H}), 1.19-1.16$ (m, 6H); ${ }^{13} \mathrm{C} \mathrm{NMR}\left(125 \mathrm{MHz}, \mathrm{CDCl}_{3}\right): \delta=170.7,159.3,136.8,130.9,129.7,129.3,128.2,116.5$, 113.9, 94.8, 81.3, 77.1, 71.2, 70.9, 65.0, 55.5, 21.3, 17.9, 15.1; IR (NaCl): v 3077, 2980, 2935, 2838, 1742, 1613 , 1586, 1514, 1457, 1401, 1374, $1301 \mathrm{~cm}^{-1}$; HRMS (ESI) m/z: $[\mathrm{M}+\mathrm{Na}]^{+}$Calcd for $\mathrm{C}_{21} \mathrm{H}_{28} \mathrm{NaO}_{6}$ 253.1199; Found 253.1201 .<smiles>[R16]OC(C)[C@@H](C=C)O[C@H]1C=C[C@@H](O)[C@@H](C)O1</smiles>

(2S,3R,6S)-6-(((3S,4R)-4-((4-methoxybenzyl)oxy)pent-1-en-3-yl)oxy)-2-methyl-3,6-dihydro-2H-pyran-3-ol (S7): A solution of $\mathbf{S 6}(2.26 \mathrm{~g}, 5.9 \mathrm{mmol})$ and $\mathrm{K}_{2} \mathrm{CO}_{3}(0.57 \mathrm{~g}, 4.1 \mathrm{mmol})$ in $\mathrm{MeOH}(57 \mathrm{ml})$ was stirred for $30 \mathrm{~min}$ at room temperature. The crude mixture was filtered with a pad of celite (rinse; Ethyl acetate $150 \mathrm{ml}$ ), then, concentrated under reduced pressure. the crude product was purified by flash column chromatography (Hexane:Ethyl acetate $=80: 20$ ) to afford $\mathbf{S 7}$ as a colorless oil $(1.74 \mathrm{~g}, 5.2 \mathrm{mmol}, 88 \%$ ).

$\mathrm{R}_{f} 0.42$ (Hexane:Ethyl acetate $\left.=60: 40\right) ;[\alpha]^{27} \mathrm{D}-54.90\left(c 0.73, \mathrm{CH}_{2} \mathrm{Cl}_{2}\right) ;{ }^{1} \mathrm{H}$ NMR $\left(500 \mathrm{MHz}, \mathrm{CDCl}_{3}\right): \delta=7.28-$ $7.26(\mathrm{~m}, 2 \mathrm{H}), 6.89-6.87(\mathrm{~m}, 2 \mathrm{H}), 5.94-5.86(\mathrm{~m}, 2 \mathrm{H}), 5.82-5.79(\mathrm{td}, J=2.18,10.18 \mathrm{~Hz}, 1 \mathrm{H}), 5.33-5.29(\mathrm{td}, J=$ $1.42,17.31 \mathrm{~Hz}, 1 \mathrm{H}), 5.22-5.19(\mathrm{td}, J=1.42,10.18 \mathrm{~Hz}, 1 \mathrm{H}), 5.18(\mathrm{~m}, 1 \mathrm{H}), 3.84-3.81(\mathrm{~m}, 4 \mathrm{H}), 3.76-3.73(\mathrm{~m}, 1 \mathrm{H})$, $3.59-3.57(\mathrm{~m}, 1 \mathrm{H}), 1.60-1.58(\mathrm{~d}, J=8.20 \mathrm{~Hz}, 1 \mathrm{H}), 1.29-1.27(\mathrm{~d}, J=6.5 \mathrm{~Hz}, 3 \mathrm{H}), 1.18-1.6(\mathrm{~d}, J=6.5 \mathrm{~Hz}, 3 \mathrm{H}) ;{ }^{13} \mathrm{C}$ $\operatorname{NMR}\left(125 \mathrm{MHz}, \mathrm{CDCl}_{3}\right): \delta=159.3,136.8,133.4,131.0,129.3,127.1,116.6,113.9,94.8,81.1,77.5,77.2,77.1$, 76.9, 70.9, 69.9, 68.2, 55.5, 17.9, 15.2; IR (NaCl): v 3439, 3079, 3041, 2976, 2934, 2899, 2837, 1613, 1513, 1457 , 1399, $1377 \mathrm{~cm}^{-1}$; HRMS (ESI) m/z: [M+Na] Calcd for $\mathrm{C}_{19} \mathrm{H}_{26} \mathrm{NaO}_{5}$ 357.1672; Found 357.1676.<smiles>C=C[C@H](O[C@H]1C=C[C@@H](OC(=O)c2ccc([N+](=O)[O-])cc2)[C@@H](C)O1)C(C)O[R16](=O)[O-]</smiles>

(2S,3S,6S)-6-(((3S,4R)-4-((4-methoxybenzyl)oxy)pent-1-en-3-yl)oxy)-2-methyl-3,6-dihydro-2H-pyran-3-yl 4-nitrobenzoate (S8): To a solution of $\mathbf{S 7}(1.74 \mathrm{~g}, 5.2 \mathrm{~mol})$ in anhydrous THF (17.3 ml), diethyl azidocarboxylate (5.2 ml, $10.4 \mathrm{~mol}, 2 \mathrm{M}$ in THF) and triphenyl phosphine $(2.80 \mathrm{~g}, 10.7 \mathrm{~mol})$ were suspended at room temperature. The solution was cooled to $0^{\circ} \mathrm{C}$. After $10 \mathrm{~min}$, p-nitrobenzoic acid $(1.74 \mathrm{~g}, 10.4 \mathrm{~mol})$ was added slowly to reaction mixture at $0{ }^{\circ} \mathrm{C}$. The resulting mixture was stirred for $30 \mathrm{~min}$ at room temperature. The reaction mixture was 
quenched with saturated $\mathrm{NaHCO}_{3}(50 \mathrm{ml})$, and then the aqueous phase was extracted with $\mathrm{Et}_{2} \mathrm{O}(4 \times 100 \mathrm{ml})$. The organic phase was washed with brine, dried over $\mathrm{Na}_{2} \mathrm{SO}_{4}$ and concentrated under reduced pressure. the crude product was purified by flash column chromatography (Hexane:Ethyl acetate $=90: 10$ ) to afford $\mathbf{S 8}$ as a white solid (2.27 g, $4.7 \mathrm{mmol}, 90 \%)$.

m.p. 93-96 ${ }^{\circ} \mathrm{C} ; \mathrm{R}_{f} 0.55$ (Hexane:Ethyl acetate $\left.=80: 20\right) ;[\alpha]^{28} \mathrm{D}+150.17\left(c 0.21, \mathrm{CH}_{2} \mathrm{Cl}_{2}\right) ;{ }^{1} \mathrm{H}$ NMR $(500 \mathrm{MHz}$, $\left.\mathrm{CDCl}_{3}\right): \delta=8.28-8.22(\mathrm{~m}, 4 \mathrm{H}), 7.27-7.25(\mathrm{~m}, 2 \mathrm{H}), 6.88-6.86(\mathrm{~m}, 2 \mathrm{H}), 6.20-6.17(\mathrm{dd}, J=5.25,9.94 \mathrm{~Hz}, 1 \mathrm{H}), 6.14-$ 6.11 (dd, $J=3.04,9.94 \mathrm{~Hz}, 1 \mathrm{H}), 5.91-5.84$ (ddd, $J=6.50,10.34,17.18 \mathrm{~Hz}, 1 \mathrm{H}), 5.32-5.29$ (m, 2H), 5.23-5.20 (td, $J=1.5,10.34 \mathrm{~Hz}, 1 \mathrm{H}), 5.17-5.15(\mathrm{dd}, J=2.51,5.25 \mathrm{~Hz}, 1 \mathrm{H}), 4.54-4.53$ (d, $J=3.04 \mathrm{~Hz}, 2 \mathrm{H}), 4.38-4.34(\mathrm{qd}, J=$ 2.34, $6.70 \mathrm{~Hz}, 1 \mathrm{H}), 4.33-4.31(\mathrm{~m}, 1 \mathrm{H}), 3.60-3.58(\mathrm{~m}, 1 \mathrm{H}), 1.25-1.223(\mathrm{~d}, J=6.70 \mathrm{~Hz}, 3 \mathrm{H}), 1.18-1.17(\mathrm{~d}, 6.70 \mathrm{~Hz}$, $3 \mathrm{H}) ;{ }^{13} \mathrm{C} \mathrm{NMR}\left(125 \mathrm{MHz}, \mathrm{CDCl}_{3}\right): \delta=164.5,159.4,150.9,136.7,135.6,131.7,131.1,131.0,129.3,125.3,123.8$, 116.8, 114.0, 94.72, 81.2, 77.1, 70.91, 67.1, 64.9, 55.5, 16.1, 15.1; IR (NaCl): v 3112, 3079, 2981, 2934, 1722, 1611, 1529, 1458, 1344, 1270, 1248, $1173 \mathrm{~cm}^{-1}$; HRMS (ESI) m/z: $[\mathrm{M}+\mathrm{Na}]^{+}$Calcd for $\mathrm{C}_{26} \mathrm{H}_{29} \mathrm{NNaO}_{8} 506.1785$; Found 506.1788.<smiles>C=C[C@H](O[C@@H]1C=C[C@@H](O)[C@@H](C)O1)C(C)O[Mg]</smiles>

(2S,3S,6S)-6-(((3S,4R)-4-((4-methoxybenzyl)oxy)pent-1-en-3-yl)oxy)-2-methyl-3,6-dihydro-2H-pyran-3-ol (4): A solution of $\mathbf{S 8}(2.06 \mathrm{~g}, 4.26 \mathrm{mmol})$ and $\mathrm{K}_{2} \mathrm{CO}_{3}(0.41 \mathrm{~g}, 2.98 \mathrm{mmol})$ in a mixed solvent $\left(\mathrm{MeOH} / \mathrm{CH}_{2} \mathrm{Cl}_{2}, \mathrm{v} / \mathrm{v}\right.$ $10 / 1,47 \mathrm{ml}$ ) was stirred for $30 \mathrm{~min}$ at room temperature. The crude mixture was filtered with a pad of celite (rinse; Ethyl acetate $150 \mathrm{ml}$ ), and then concentrated under reduced pressure. the crude product was purified by flash column chromatography (Hexane:Ethyl acetate $=80: 20)$ to afford $\mathbf{4}$ as a colorless oil $(1.31 \mathrm{~g}, 3.92 \mathrm{mmol}, 92 \%)$. $\mathrm{R}_{f} 0.43$ (Hexane:Ethyl acetate $\left.=60: 40\right) ;[\alpha]^{28} \mathrm{D}+52.86\left(c 0.61, \mathrm{CH}_{2} \mathrm{Cl}_{2}\right) ;{ }^{1} \mathrm{H}$ NMR $\left(500 \mathrm{MHz}, \mathrm{CDCl}_{3}\right): \delta=7.26-$ $7.24(\mathrm{~m}, 2 \mathrm{H}), 6.87-6.85(\mathrm{~m}, 2 \mathrm{H}), 6.18-6.15(\mathrm{dd}, J=5.55,10.01 \mathrm{~Hz}, 1 \mathrm{H}), 5.93-5.9 \mathrm{H} 0(\mathrm{dd}, J=3.11,10.01 \mathrm{~Hz}, 1 \mathrm{H})$, $5.90-5.83$ (ddd, $J=6.39,10.49,17.29 \mathrm{~Hz}, 1 \mathrm{H}), 5.30-5.26(\mathrm{td}, J=1.55,17.32 \mathrm{~Hz}, 1 \mathrm{H}), 5.20-5.18(\mathrm{~m}, 2 \mathrm{H}), 4.52$ (s, $2 \mathrm{H}), 4.29-4.27(\mathrm{~m}, 1 \mathrm{H}), 4.16-4.11(\mathrm{qd}, J=2.02,6.68 \mathrm{~Hz}, 1 \mathrm{H}), 3.80(\mathrm{~s}, 3 \mathrm{H}), 3.60-3.54(\mathrm{~m}, 2 \mathrm{H}), 1.42-1.40(\mathrm{~d}, J=$ $10.49 \mathrm{~Hz}, 1 \mathrm{H}), 1.24-1.23(\mathrm{~d}, J=6.39 \mathrm{~Hz}, 3 \mathrm{H}), 1.16-1.15(\mathrm{~d}, J=6.39,3 \mathrm{H}) ;{ }^{13} \mathrm{C}$ NMR $\left(125 \mathrm{MHz}, \mathrm{CDCl}_{3}\right): \delta=$ 159.4, 136.8, 131.0, 130.4, 129.3, 128.7, 116.7, 114.0, 94.9, 80.8, 77.1, 70.9, 66.6, 64.4, 55.5, 16.0, 15.2; IR $(\mathrm{NaCl}): v 3442,3077,3041,2979,2934,2902,1613,1586,1513,1458,1403,1378 \mathrm{~cm}^{-1} ;$ HRMS (ESI) m/z: $[\mathrm{M}+\mathrm{Na}]^{+}$Calcd for $\mathrm{C}_{19} \mathrm{H}_{26} \mathrm{NaO}_{5} 357.1672$; Found 357.1674.

\section{Asymmetric addition of alcohol 4 to allenes 3 via palladium catalyst and Ring Closing}

\section{Metathesis}

\section{- Hydroalkoxylation between alcohol 4 and allene 3 [(S,S) ligand]}
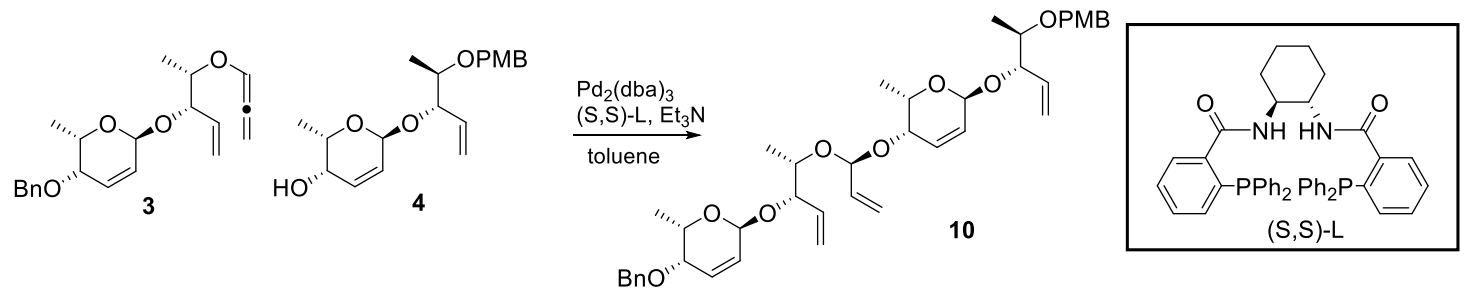


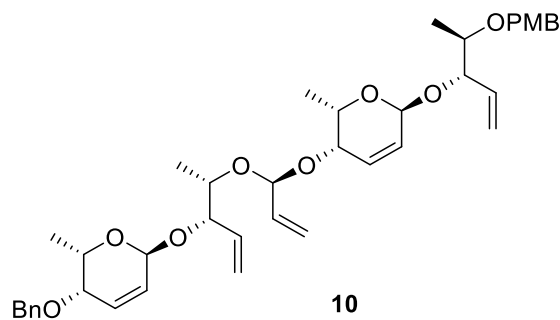

(2S,3S,6S)-3-(benzyloxy)-6-(((3S,4S)-4-(((S)-1-(((2S,3S,6S)-6-(((3S,4R)-4-((4-methoxybenzyl)oxy)pent-1-en -3-yl)oxy)-2-methyl-3,6-dihydro-2H-pyran-3-yl)oxy)allyl)oxy)pent-1-en-3-yl)oxy)-2-methyl-3,6-dihydro-2 H-pyran (10): Alcohol $4(535.0 \mathrm{mg}, 1.600 \mathrm{mmol})$ was reacted with $\mathrm{Pd}_{2}\left(\mathrm{dba}_{3}(18.3 \mathrm{mg}, 0.020 \mathrm{mmol}),(S, S)-\mathrm{L}\right.$ $(27.6 \mathrm{mg}, 0.040 \mathrm{mmol})$, ene-allene $3(273.9 \mathrm{mg}, 0.800 \mathrm{mmol})$ and triethylamine $(11.2 \mu \mathrm{L}, 0.080 \mathrm{mmol})$ in toluene $(1.6 \mathrm{ml})$ under nitrogen. The reaction mixture was stirred for $2 \mathrm{~h}$ at $40{ }^{\circ} \mathrm{C}$. The crude mixture was concentrated under reduced pressure. the crude product was purified by flash column chromatography (Hexane:Ethyl acetate = 90:10) to afford 10 as a colorless oil (509.0 $\mathrm{mg}, 0.754 \mathrm{mmol}, 94 \%$ yield).

$\mathrm{R}_{f} 0.43$ (Hexane:Ethyl acetate $\left.=80: 20\right) ;[\alpha]^{29}{ }_{\mathrm{D}}+78.25\left(c 0.82, \mathrm{CH}_{2} \mathrm{Cl}_{2}\right) ;{ }^{1} \mathrm{H} \mathrm{NMR}\left(500 \mathrm{MHz}, \mathrm{CDCl}_{3}\right): \delta=7.34-$ $7.23(\mathrm{~m}, 7 \mathrm{H}), 6.87-6.85(\mathrm{~m}, 2 \mathrm{H}), 6.18-6.15(\mathrm{dd}, J=5.41,10.08 \mathrm{~Hz}, 1 \mathrm{H}), 6.14-6.11(\mathrm{dd}, J=5.20,10.04 \mathrm{~Hz}, 1 \mathrm{H})$, 5.97-5.92 (m, 2H), 5.90-5.80 (m, 3H), 5.40-5.37 (d, $J=17.37 \mathrm{~Hz}, 1 \mathrm{H}), 5.29-5.17(\mathrm{~m}, 7 \mathrm{H}), 5.02-5.01(\mathrm{~d}, J=5.73$ $\mathrm{Hz}, 1 \mathrm{H}), 4.65-4.54(\mathrm{dd}, J=11.85,42.44 \mathrm{~Hz}, 2 \mathrm{H}), 4.51(\mathrm{~s}, 2 \mathrm{H}), 4.30-4.27$ (m, 1H), 4.16-4.09 (m, 3H), 3.79-3.76 $(\mathrm{m}, 4 \mathrm{H}), 3.71-3.69(\mathrm{dd}, J=2.41,5.39 \mathrm{~Hz}, 1 \mathrm{H}), 3.58-3.54(\mathrm{qd}, J=3.39,6.30 \mathrm{~Hz}, 1 \mathrm{H}), 3.51-3.50(\mathrm{dd}, J=2.72$, $5.49 \mathrm{~Hz}, 1 \mathrm{H}), 1.28-1.27(\mathrm{~d}, J=6.63 \mathrm{~Hz}, 3 \mathrm{H}), 1.22-1.21(\mathrm{~d}, J=6.59 \mathrm{~Hz}, 3 \mathrm{H}), 1.15-1.14(\mathrm{~d}, J=6.43 \mathrm{~Hz}, 3 \mathrm{H}), 1.08-$ $1.07(\mathrm{~d}, J=6.29 \mathrm{~Hz}, 3 \mathrm{H}) ;{ }^{13} \mathrm{C} \mathrm{NMR}\left(125 \mathrm{MHz}, \mathrm{CDCl}_{3}\right): \delta=159.3,138.9,136.8,136.3,136.1,131.0,129.7,129.3$, 129.2, 128.7, 128.5, 127.9, 127.8, 127.7, 118.4, 117.3, 116.6, 113.9, 102.7, 94.9, 94.7, 81.5, 80.4, 72.7, 70.9, 70.8, 69.6, 68.7, 66.7, 66.3, 55.5, 16.8, 16.3, 16.1, 15.3; IR (NaCl): v 3081, 3034, 2980, 2934, 2896, 2837, 1613, 1586, 1513, 1454, 1403, $1378 \mathrm{~cm}^{-1}$; HRMS (ESI) m/z: [M+Na] $]^{+}$Calcd for $\mathrm{C}_{40} \mathrm{H}_{52} \mathrm{NaO}_{9}$ 699.3504; Found 699.3508 .

\section{- Ring Closing Metathesis of O,O-acetal compound 10}

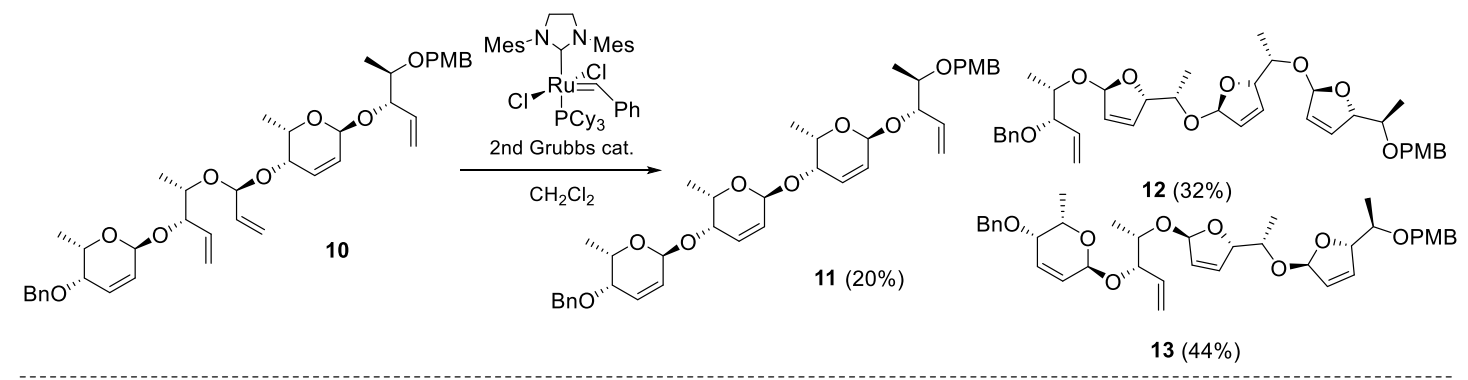

The O,O-acetal compound 10 (105.6 mg, $0.156 \mathrm{mmol})$ was reacted with catalyst GII (13.2 $\mathrm{mg}, 0.016 \mathrm{mmol})$ in $\mathrm{CH}_{2} \mathrm{Cl}_{2}(1.56 \mathrm{ml})$. The reaction mixture was stirred for $17 \mathrm{~h}$ at $40^{\circ} \mathrm{C}$. then, concentrated under reduced pressure. the crude product was purified by flash column chromatography (Hexane:Ethyl acetate $=80: 20$ ) to afford $\mathbf{1 1}$ as a white amorphos solid (20.2 mg, $0.031 \mathrm{mmol}), 12$ as a colorless oil $(32.4 \mathrm{mg}, 0.050 \mathrm{mmol})$ and 13 as a colorless oil (44.5 mg,0.069 mmol).

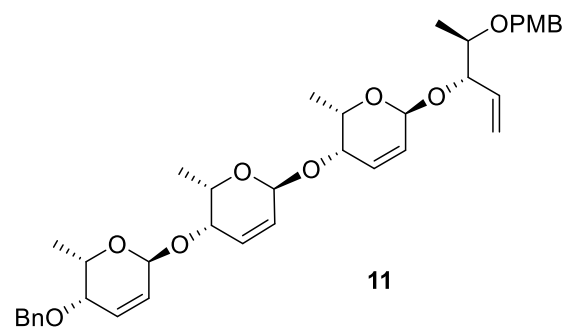


$(2 S, 3 S, 6 S)-3-($ benzyloxy)-6-(((2S,3S,6S)-6-(((2S,3S,6S)-6- $(((3 S, 4 R)-4-((4-m e t h o x y b e n z y l) o x y) p e n t-1-e n-3-y$ l)oxy)-2-methyl-3,6-dihydro-2H-pyran-3-yl)oxy)-2-methyl-3,6-dihydro-2H-pyran-3-yl)oxy)-2-methyl-3,6-d ihydro-2H-pyran (11): m.p. $157-160^{\circ} \mathrm{C} ; \mathrm{R}_{f} 0.29$ (Hexane:Ethyl acetate $\left.=70: 30\right) ;[\alpha]^{27} \mathrm{D}+155.26(c 0.45$, $\left.\mathrm{CH}_{2} \mathrm{Cl}_{2}\right) ;{ }^{1} \mathrm{H}$ NMR $\left(500 \mathrm{MHz}, \mathrm{CDCl}_{3}\right): \delta=7.36-7.23(\mathrm{~m}, 7 \mathrm{H}), 6.87-6.84(\mathrm{~m}, 2 \mathrm{H}), 6.15-6.08(\mathrm{~m}, 3 \mathrm{H}), 6.03-6.00$ $(\mathrm{m}, 1 \mathrm{H}), 5.95-5.91(\mathrm{~m}, 2 \mathrm{H}), 5.88-5.82(\mathrm{ddd}, J=6.44,10.62,17.13 \mathrm{~Hz}, 1 \mathrm{H}), 5.29-5.16(\mathrm{~m}, 3 \mathrm{H}), 5.07-5.02(\mathrm{~m}, 2 \mathrm{H})$, 4.65-4.54 (dd, $J=12.12,41.57 \mathrm{~Hz}, 2 \mathrm{H}), 4.51(\mathrm{~s}, 2 \mathrm{H}), 4.30-4.28(\mathrm{~m}, 1 \mathrm{H}), 4.20-4.12(\mathrm{~m}, 3 \mathrm{H}), 3.80(\mathrm{~s}, 3 \mathrm{H}), 3.65-$ $3.63(\mathrm{dd}, J=2.12,5.38 \mathrm{~Hz}, 2 \mathrm{H}), 3.58-3.53(\mathrm{qd}, J=3.54,6.35 \mathrm{~Hz}, 1 \mathrm{H}), 3.49-3.48(\mathrm{dd}, J=2.45,4.99 \mathrm{~Hz}, 1 \mathrm{H})$, $1.34-1.33(\mathrm{~d}, J=6.66 \mathrm{~Hz}, 3 \mathrm{H}), 1.29-1.27(\mathrm{~d}, J=6.37 \mathrm{~Hz}, 3 \mathrm{H}), 1.22-1.21(\mathrm{~d}, J=6.66 \mathrm{~Hz}, 3 \mathrm{H}), 1.15-1.14(\mathrm{~d}, J=$ $6.41 \mathrm{~Hz}, 3 \mathrm{H}) ;{ }^{13} \mathrm{C}$ NMR $\left(125 \mathrm{MHz}, \mathrm{CDCl}_{3}\right): \delta=159.3,138.8,136.8,131.0,129.6,129.3,129.3,129.1,128.6$, $127.9,127.9,127.8,116.6,114.0,96.3,96.2,94.7,80.3,77.1,71.0,70.9,70.6,70.5,69.6,66.6,65.9,65.8,55.5$, 16.6, 16.4, 16.1, 15.2; IR (NaCl): v 3068, 3034, 2972, 2917, 2850, 1614, 1513, 1456, 1399, 1301, 1248, $1192 \mathrm{~cm}^{-}$ ${ }^{1}$; HRMS (ESI) m/z: [M+Na] $]^{+}$Calcd for $\mathrm{C}_{38} \mathrm{H}_{48} \mathrm{NaO}_{9}$ 671.3191; Found 671.3193 .

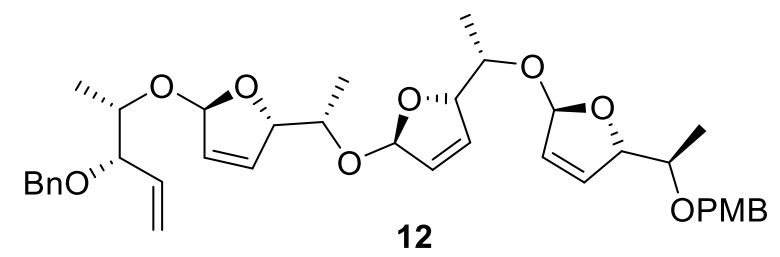

$(2 S, 5 S)-2-(((2 S, 3 S)-3-($ benzyloxy)pent-4-en-2-yl)oxy)-5- $((S)-1-(((2 S, 5 S)-5-((S)-1-(((2 S, 5 S)-5-((R)-1-((4-m e t h$ oxybenzyl)oxy)ethyl)-2,5-dihydrofuran-2-yl)oxy)ethyl)-2,5-dihydrofuran-2-yl)oxy)ethyl)-2,5-dihydrofuran (12): $\mathrm{R}_{f} 0.50$ (Hexane:Ethyl acetate $\left.=70: 30\right) ;[\alpha]^{27} \mathrm{D}-190.38\left(c 0.32, \mathrm{CH}_{2} \mathrm{Cl}_{2}\right) ;{ }^{1} \mathrm{H}$ NMR $\left(500 \mathrm{MHz}, \mathrm{CDCl}_{3}\right): \delta=$ 7.36-7.23 (m, 7H), 6.87-6.85 (m, 2H), 6.24-6.22 (td, $J=1.24-6.06 \mathrm{~Hz}, 1 \mathrm{H}), 6.18-6.14(\mathrm{~m}, 2 \mathrm{H}), 5.94-5.91(\mathrm{~m}, 3 \mathrm{H})$, 5.87-.79 (m, 4H), 5.32-5.30 (m, 1H), 5.29-5.28 (m, 1H), 5.10-5.06 (m, 2H), 4.65-4.41 (m, 4H), 4.01-43.93 (m, $3 \mathrm{H}), 3.92-3.87$ (qd, $J=6.37,6.37 \mathrm{~Hz}, 1 \mathrm{H}), 3.79(\mathrm{~s}, 3 \mathrm{H}), 3.51-3.47(\mathrm{qd}, J=6.21,6.37 \mathrm{~Hz}, 1 \mathrm{H}), 1.18-1.17(\mathrm{~d}, J=$ $6.28 \mathrm{~Hz}, 3 \mathrm{H}), 1.16-1.14(\mathrm{~d}, J=6.43 \mathrm{~Hz}, 3 \mathrm{H}), 1.04-1.03(\mathrm{~d}, J=6.37 \mathrm{~Hz}, 3 \mathrm{H}), 1.02-1.01(\mathrm{~d}, J=6.37 \mathrm{~Hz}, 3 \mathrm{H}) ;{ }^{13} \mathrm{C}$ $\operatorname{NMR}\left(125 \mathrm{MHz}, \mathrm{CDCl}_{3}\right): \delta=159.4,139.1,135.2,133.4,133.3,133.0,131.0,129.4,128.4,128.2,128.0,127.9$, $127.8,127.5,118.5,114.0,108.1,108.0,108.0,88.4,87.4,87.3,82.7,76.5,74.4,73.8,73.7,71.2,70.9,55.5,16.4$, 16.3, 15.4, 15.3; IR (NaCl): v 3087, 3031, 2976, 2931, 2874, 1613, 1513, 1454, 1375, 1339, 1300, $1248 \mathrm{~cm}^{-1}$; HRMS (ESI) m/z: [M+Na] $]^{+}$Calcd for $\mathrm{C}_{38} \mathrm{H}_{48} \mathrm{NaO}_{9}$ 671.3191; Found 671.3192.<smiles>C=C[C@H](O[C@H]1C=C[C@@H](OCc2ccccc2)[C@@H](C)O1)[C@@H](C)OC1C=CC([C@@H](C)OC2C=CC(C(C)O[R16](=O)c3ccccc3)O2)O1</smiles>

$(2 S, 3 S, 6 S)-3-($ benzyloxy)-6-(((3S,4S)-4-(((2S,5S)-5-((S)-1-(((2S,5S)-5-((R)-1-((4-methoxybenzyl)oxy)ethyl)2,5-dihydrofuran-2-yl)oxy)ethyl)-2,5-dihydrofuran-2-yl)oxy)pent-1-en-3-yl)oxy)-2-methyl-3,6-dihydro-2H -pyran (13): $\mathrm{R}_{f} 0.47($ Hexane:Ethyl acetate $=70: 30) ;[\alpha]^{27} \mathrm{D}-49.12\left(c 0.82, \mathrm{CH}_{2} \mathrm{Cl}_{2}\right) ;{ }^{1} \mathrm{H}$ NMR $\left(500 \mathrm{MHz}, \mathrm{CDCl}_{3}\right)$ : $\delta=7.34-7.23(\mathrm{~m}, 7 \mathrm{H}), 6.87-6.85(\mathrm{~m}, 2 \mathrm{H}), 6.24-6.23(\mathrm{td}, J=1.23,6.02 \mathrm{~Hz}, 1 \mathrm{H}), 6.16-6.14(\mathrm{td}, J=1.22,6.05 \mathrm{~Hz}$, $1 \mathrm{H}), 6.12-6.09(\mathrm{~m}, 1 \mathrm{H}), 6.05-6.02(\mathrm{~m}, 1 \mathrm{H}), 5.95-5.94(\mathrm{td}, J=1.01,4.23 \mathrm{~Hz}, 1 \mathrm{H}), 5.93-5.92(\mathrm{td}, J=1.04,4.25 \mathrm{~Hz}$, $1 \mathrm{H}), 5.87-.80(\mathrm{~m}, 3 \mathrm{H}), 5.32-5.28(\mathrm{td}, J=1.93,17.14 \mathrm{~Hz}, 1 \mathrm{H}), 5.25-5.24(\mathrm{~d}, J=3.00 \mathrm{~Hz}, 1 \mathrm{H}), 5.19-5.16(\mathrm{~m}, 1 \mathrm{H})$, 5.10-5.07 (m, 1H), 4.82-4.79 (m, 1H), 4.64-4.45 (m, 4H), 4.22-4.19 (t, $J=6.61 \mathrm{~Hz}, 1 \mathrm{H}), 4.14-4.10(\mathrm{qd}, J=2.67$, $6.57 \mathrm{~Hz}, 1 \mathrm{H}), 4.03-3.98$ (qd, $J=4.61,6.60 \mathrm{~Hz}, 1 \mathrm{H}), 3.84-3.78$ (m , 4H), 3.51-3.46 (m, 2H), 1.28-1.27 (d, $J=6.67$ 
$\mathrm{Hz}, 3 \mathrm{H}), 1.19-1.17(\mathrm{~d}, J=6.39 \mathrm{~Hz}, 3 \mathrm{H}), 1.12-1.10(\mathrm{~d}, J=6.39 \mathrm{~Hz}, 3 \mathrm{H}), 1.03-1.02(\mathrm{~d}, J=6.32 \mathrm{~Hz}, 3 \mathrm{H}) ;{ }^{13} \mathrm{C} \mathrm{NMR}$ $\left(125 \mathrm{MHz} \mathrm{CDCl}_{3}\right): \delta=159.3,139.0,136.1,133.4,132.9,131.0,130.1,129.4,128.5,128.2,127.9,127.7,127.5$, $116.9,114.0,108.0,107.9,95.2,88.4,87.3,81.5,76.5,74.9,73.7,71.2,70.7,69.8,66.6,55.5,16.6,16.4,16.2$, 15.3; IR (NaCl): v 3087, 3033, 2977, 2932, 2896, 1613, 1513, 1454, 1376, 1341, 1301, $1248 \mathrm{~cm}^{-1}$; HRMS (ESI) $\mathrm{m} / \mathrm{z}:[\mathrm{M}+\mathrm{Na}]^{+}$Calcd for $\mathrm{C}_{38} \mathrm{H}_{48} \mathrm{NaO}_{9}$ 671.3191; Found 671.3191.

\section{- Reversibility test of compound 11}

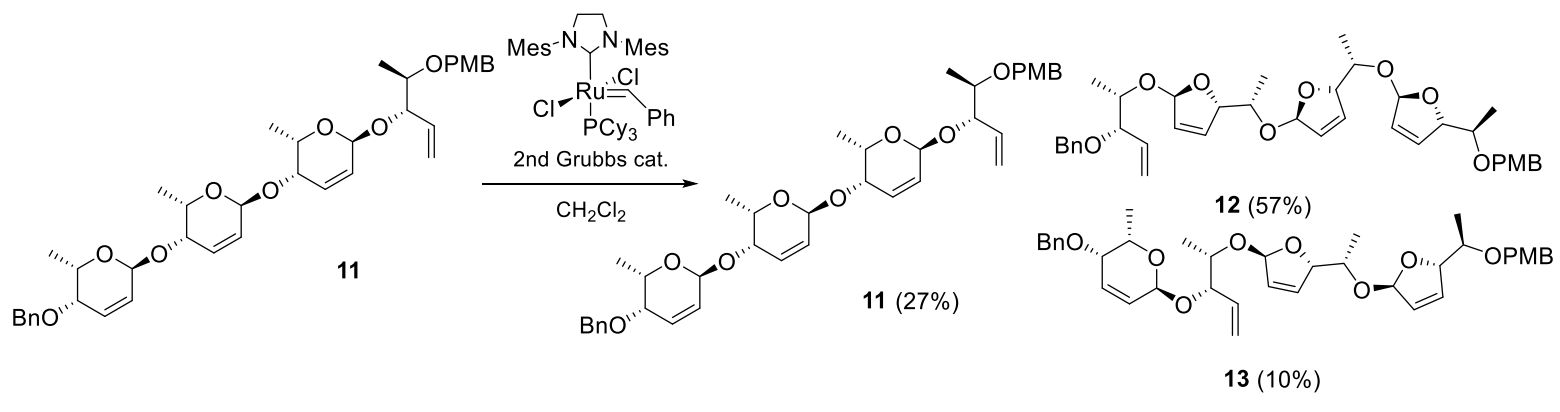

The O,O-acetal compound $11(59.0 \mathrm{mg}, 0.091 \mathrm{mmol})$ was reacted with catalyst GII $(7.7 \mathrm{mg}, 0.009 \mathrm{mmol})$ in $\mathrm{CH}_{2} \mathrm{Cl}_{2}(0.91 \mathrm{ml})$. The reaction mixture was stirred for $19 \mathrm{~h}$ at $40^{\circ} \mathrm{C}$. then, concentrated under reduced pressure. the crude product was purified by flash column chromatography (Hexane:Ethyl acetate $=80: 20$ ) to afford $\mathbf{1 1}$ as a white amorphos solid (15.9 mg, $0.025 \mathrm{mmol}), \mathbf{1 2}$ as a colorless oil (33.7 $\mathrm{mg}, 0.052 \mathrm{mmol})$ and $\mathbf{1 3}$ as a colorless oil (5.9 mg, $0.009 \mathrm{mmol})$

\section{- Hydroalkoxylation between alcohol 3 and allene 2 [(R,R) ligand]}

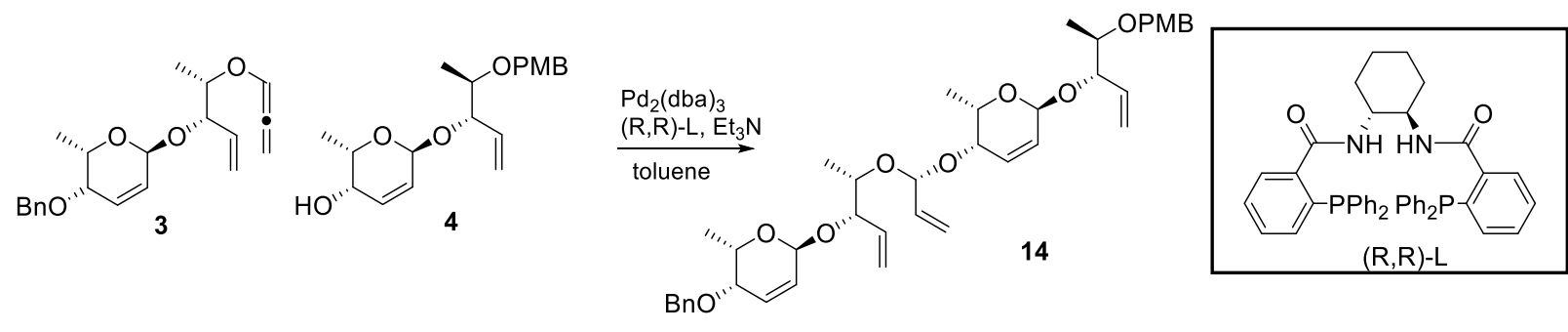

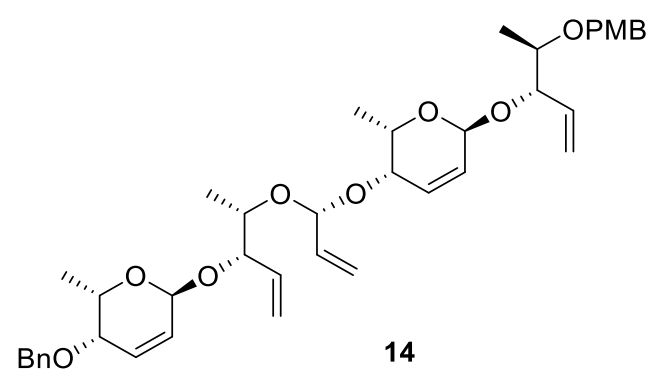

$(2 S, 3 S, 6 S)-3-($ benzyloxy)-6-(((3S,4S)-4-(((R)-1-(((2S,3S,6S)-6-(((3S,4R)-4-((4-methoxybenzyl)oxy)pent-1en-3-yl)oxy)-2-methyl-3,6-dihydro-2H-pyran-3-yl)oxy)allyl)oxy)pent-1-en-3-yl)oxy)-2-methyl-3,6-dihydro- 
2H-pyran (14): Alcohol 4 (167.2 mg, $0.500 \mathrm{mmol})$ was reacted with $\mathrm{Pd}_{2}(\mathrm{dba})_{3}(22.9 \mathrm{mg}, 0.025 \mathrm{mmol}),(R, R)-\mathrm{L}$ (34.5 mg, $0.050 \mathrm{mmol})$, ene-allene $3(85.9 \mathrm{mg}, 0.250 \mathrm{mmol})$ and triethylamine $(3.5 \mu \mathrm{L}, 0.025 \mathrm{mmol})$ in toluene $(0.5 \mathrm{ml})$ under nitrogen. The reaction mixture was stirred for $2 \mathrm{~h}$ at $40^{\circ} \mathrm{C}$. The crude mixture was concentrated under reduced pressure. The crude product was purified by flash column chromatography (Hexane:Ethyl acetate $=90: 10)$ to afford $\mathbf{1 4}$ as a colorless oil ( $145.0 \mathrm{mg}, 0.214 \mathrm{mmol}, 86 \%$ yield).

$\mathrm{R}_{f} 0.42$ (Hexane:Ethyl acetate $\left.=80: 20\right) ;[\alpha]^{27} \mathrm{D}+95.04\left(c 0.565, \mathrm{CH}_{2} \mathrm{Cl}_{2}\right) ;{ }^{1} \mathrm{H} \mathrm{NMR}\left(600 \mathrm{MHz}, \mathrm{CDCl}_{3}\right): \delta=7.33-$ $7.23(\mathrm{~m}, 7 \mathrm{H}), 6.87-6.85(\mathrm{~m}, 2 \mathrm{H}), 6.13-6.10(\mathrm{dd}, J=5.21,9.95 \mathrm{~Hz}, 2 \mathrm{H}), 5.96-5.91(\mathrm{~m}, 2 \mathrm{H}), 5.89-5.76(\mathrm{~m}, 3 \mathrm{H})$, $5.43-5.39(\mathrm{~d}, J=17.43 \mathrm{~Hz}, 1 \mathrm{H}), 5.30-5.16(\mathrm{~m}, 8 \mathrm{H}), 4.64-4.53(\mathrm{dd}, J=12.05,41.35 \mathrm{~Hz}, 2 \mathrm{H}), 4.51(\mathrm{~s}, 2 \mathrm{H}), 4.31-$ $4.28(\mathrm{~m}, 1 \mathrm{H}), 4.13-4.04(\mathrm{~m}, 3 \mathrm{H}), 3.79(\mathrm{~s}, 3 \mathrm{H}), 3.79-3.74(\mathrm{~m}, 2 \mathrm{H}), 3.66-3.65(\mathrm{dd}, J=2.48,5.30 \mathrm{~Hz}, 1 \mathrm{H}), 3.58-3.53$ $(\mathrm{qd}, J=3.49,6.22 \mathrm{~Hz}, 1 \mathrm{H}), 3.51-3.49(\mathrm{dd}, J=2.70,5.15 \mathrm{~Hz}, 1 \mathrm{H}), 1.27-1.25(\mathrm{~d}, J=6.60 \mathrm{~Hz}, 3 \mathrm{H}), 1.23-1.21(\mathrm{~d}, J$ $=6.60 \mathrm{~Hz}, 3 \mathrm{H}), 1.16-1.15(\mathrm{~d}, J=6.60 \mathrm{~Hz}, 3 \mathrm{H}), 1.16-1.14(\mathrm{~d}, J=6.40 \mathrm{~Hz}, 3 \mathrm{H}) ;{ }^{13} \mathrm{C}$ NMR $\left(150 \mathrm{MHz}, \mathrm{CDCl}_{3}\right): \delta=$ $159.3,138.9,136.8,136.2,136.2,131.0,129.7,129.3,129.2,128.6,128.5,127.9,127.8,118.6,117.3,116.6$, $113.9,102.1,95.2,94.7,82.3,80.3,75.5,70.8,69.6,66.6,66.2,66.3,65.8,55.5,17.5,16.5,16.1,15.3 ;$ IR (NaCl): v 3077, 3032, 2980, 2934, 2898, 1646, 1613, 1513, 1454, 1403, 1377, $1342 \mathrm{~cm}^{-1}$; HRMS (ESI) m/z: [M+Na] Calcd for $\mathrm{C}_{40} \mathrm{H}_{52} \mathrm{NaO}_{9}$ 699.3504; Found 699.3508.

\section{- Ring Closing Metathesis of O,O-acetal compound 14}

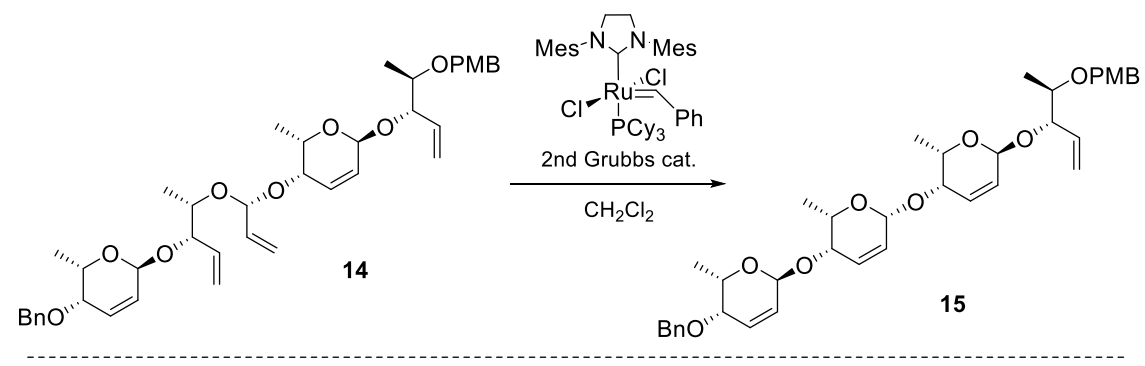

Following the general procedure, the O,O-acetal compound $14(102.5 \mathrm{mg}, 0.151 \mathrm{mmol})$ was reacted with catalyst GII $(6.4 \mathrm{mg}, 0.008 \mathrm{mmol})$ in $\mathrm{CH}_{2} \mathrm{Cl}_{2}(1.50 \mathrm{ml})$. The reaction mixture was stirred for $24 \mathrm{~h}$ at $40{ }^{\circ} \mathrm{C}$. Then, concentrated under reduced pressure. The crude mixture was recrystalized by $\mathrm{CH}_{2} \mathrm{Cl}_{2} / \mathrm{Hexane}$ to afford $\mathbf{1 5}$ as a white solid ( $25.8 \mathrm{mg}, 0.040 \mathrm{mmol}, 26 \%$ yield).

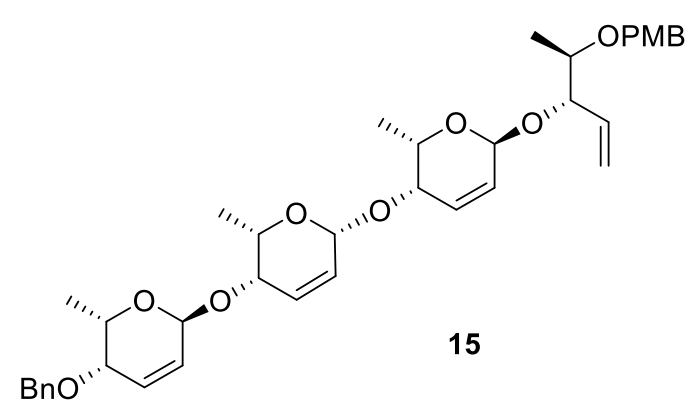

$(2 S, 3 S, 6 S)-3-($ benzyloxy)-6-(((2S,3S,6S)-6-(((2S,3S,6S)-6-(((3S,4R)-4-((4-methoxybenzyl)oxy)pent-1-en-3yl)oxy)-2-methyl-3,6-dihydro-2H-pyran-3-yl)oxy)-2-methyl-3,6-dihydro-2H-pyran-3-yl)oxy)-2-methyl-3,6dihydro-2H-pyran (15): m.p. 157- $159^{\circ} \mathrm{C} ; \mathrm{R}_{f} 0.28$ (Hexane:Ethyl acetate $\left.=70: 30\right) ;[\alpha]^{27} \mathrm{D}+80.98(c 0.200$, $\mathrm{CH}_{2} \mathrm{Cl}_{2}$ ); ${ }^{1} \mathrm{H}$ NMR $\left(500 \mathrm{MHz}, \mathrm{CDCl}_{3}\right): \delta=7.34-7.23(\mathrm{~m}, 7 \mathrm{H}), 6.87-6.84(\mathrm{~m}, 2 \mathrm{H}), 6.27-6.24(\mathrm{dd}, J=5.15,10.10$ $\mathrm{Hz}, 1 \mathrm{H}$ ), 6.14-6.11 (dd, $J=5.20,10.05 \mathrm{~Hz}, 1 \mathrm{H}), 6.07-6.04$ (ddd, $J=1.44,4.55,10.20 \mathrm{~Hz}, 1 \mathrm{H}$ ), 6.01-5.97 (ddd, $J$ $=2.94,6.60,9.65 \mathrm{~Hz}, 2 \mathrm{H}), 5.89-5.82(\mathrm{ddd}, J=6.53,10.57,17.18 \mathrm{~Hz}, 1 \mathrm{H}), 5.81-5.79(\mathrm{~d}, J=10.21 \mathrm{~Hz}, 1 \mathrm{H}), 5.29-$

\section{S12}


$5.25(\mathrm{td}, J=1.38,17.17 \mathrm{~Hz}, 1 \mathrm{H}), 5.24-5.22(\mathrm{~m}, 2 \mathrm{H}), 5.19-5.16(\mathrm{td}, J=1.29,10.52 \mathrm{~Hz}, 1 \mathrm{H}), 5.07-5.06(\mathrm{~d}, J=2.79$ $\mathrm{Hz}, 1 \mathrm{H}), 5.03-5.02$ (d, $J=2.88 \mathrm{~Hz}, 1 \mathrm{H}), 4.66-4.54$ (dd, $J=12.06,41.72 \mathrm{~Hz}, 2 \mathrm{H}), 4.51(\mathrm{~s}, 2 \mathrm{H}), 4.30-4.28(\mathrm{~m}, 1 \mathrm{H})$, 4.18-4.09 (m, 2H), 3.88-3.87 (dd, $J=2.59,5.61 \mathrm{~Hz}, 1 \mathrm{H}) 3.84-3.77(\mathrm{~m}, 5 \mathrm{H}), 3.58-3.53(\mathrm{qd}, J=3.43,6.31 \mathrm{~Hz}, 1 \mathrm{H})$, $3.49-3.48$ (dd, $J=2.76,5.34 \mathrm{~Hz}, 1 \mathrm{H}), 1.34-1.33$ (d, $J=6.78 \mathrm{~Hz}, 3 \mathrm{H}), 1.29-1.27$ (d, $J=6.42 \mathrm{~Hz}, 3 \mathrm{H}), 1.23-1.21$ $(\mathrm{d}, J=6.60 \mathrm{~Hz}, 3 \mathrm{H}), 1.16-1.14(\mathrm{~d}, J=6.42 \mathrm{~Hz}, 3 \mathrm{H}) ;{ }^{13} \mathrm{C} \mathrm{NMR}\left(125 \mathrm{MHz}, \mathrm{CDCl}_{3}\right): \delta=159.1,138.8,136.8,131.1$, $130.7,130.4,129.5,129.3,129.3,128.5,128.5,127.9,127.8,127.7,116.6,113.9,96.0,94.8,94.5,80.4,71.4$, 71.1, 70.8, 70.3, 69.6, 66.6, 66.2, 55.5, 16.7, 16.5, 16.4, 15.3; IR (NaCl): v 3068, 3035, 2984, 2973, 2931, 2905 , 2836, 1614, 1514, 1455, 1400, 1335, $1302 \mathrm{~cm}^{-1}$; HRMS (ESI) m/z: $[\mathrm{M}+\mathrm{Na}]^{+}$Calcd for $\mathrm{C}_{38} \mathrm{H}_{48} \mathrm{NaO}_{9} 671.3191$; Found 671.3194.

\section{Synthesis of ene-allene 16}

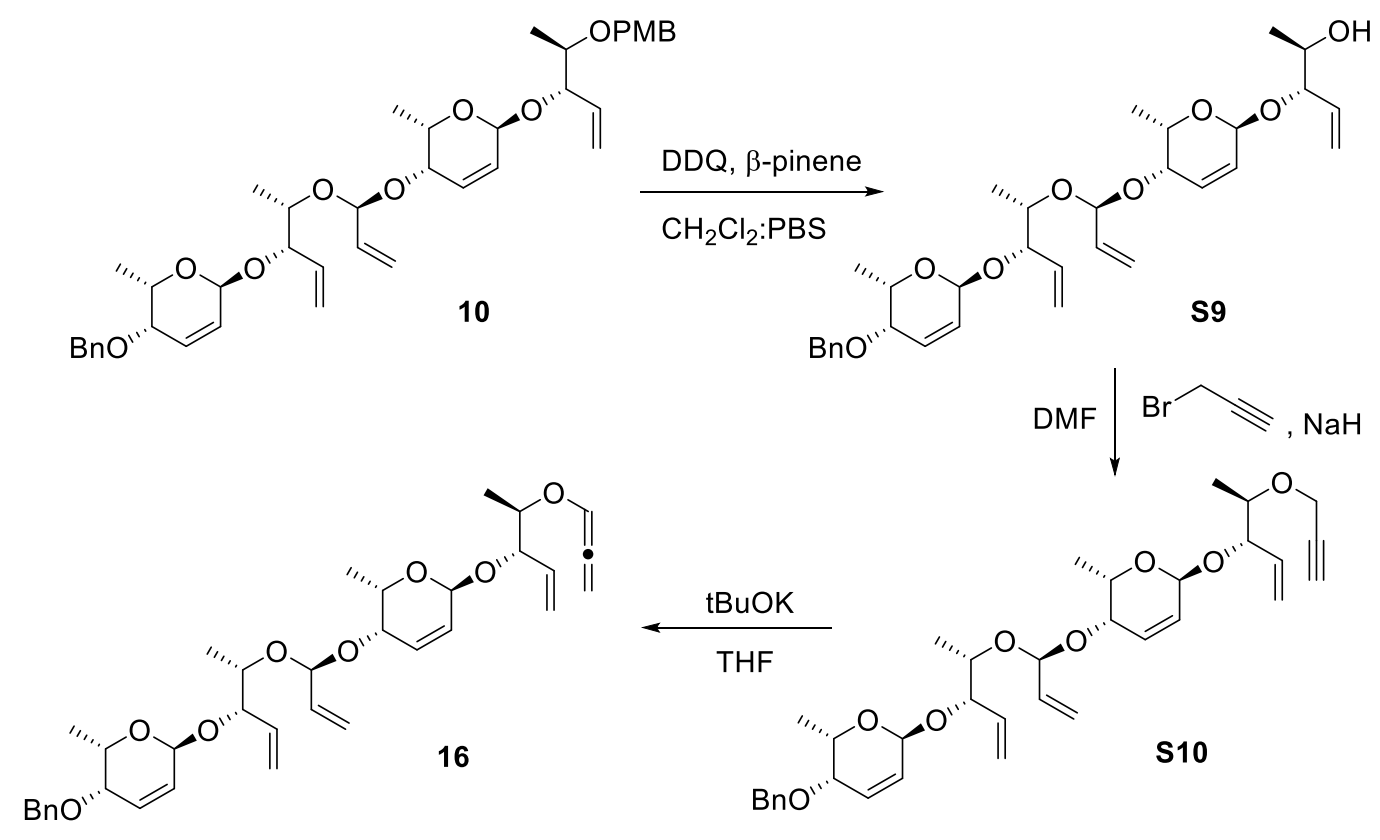

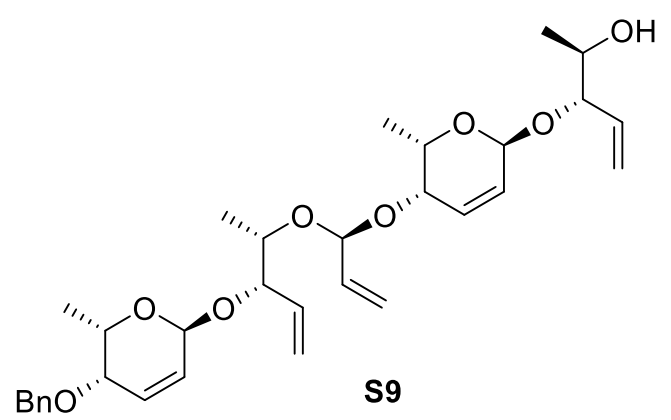

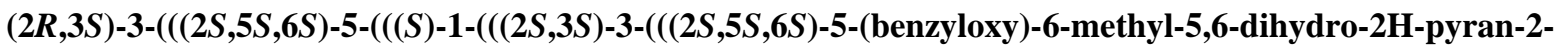
yl)oxy)pent-4-en-2-yl)oxy)allyl)oxy)-6-methyl-5,6-dihydro-2H-pyran-2-yl)oxy)pent-4-en-2-ol (S9): To a solution of $10(676.8 \mathrm{mg}, 1.00 \mathrm{mmol})$ in a mixed solvent $\left(\mathrm{CH}_{2} \mathrm{Cl}_{2} / \mathrm{pH} 7.0\right.$ phosphate buffer, v/v 20/1, $\left.100 \mathrm{ml}\right)$ at $0{ }^{\circ} \mathrm{C}$ was added DDQ $(454.0 \mathrm{mg}, 2.00 \mathrm{mmol})$. After stirring at $0{ }^{\circ} \mathrm{C}$ for $1 \mathrm{~h}$, the reaction was quenched by sat. $\mathrm{NaHCO}_{3}(30 \mathrm{ml})$, and then the aqueous phase was extracted with $\mathrm{CH}_{2} \mathrm{Cl}_{2}(4 \times 50 \mathrm{ml})$. The organic phase was 
washed with brine, dried over $\mathrm{Na}_{2} \mathrm{SO}_{4}$ and concentrated under reduced pressure. the crude product was purified by flash column chromatography (Hexane:Ethyl acetate $=70: 30)$ to afford $\mathbf{S 9}$ as a colorless oil $(457.9 \mathrm{mg}, 0.83$ mmol, $83 \%$ yield)

$\mathrm{R}_{f} 0.55$ (Hexane:Ethyl acetate $\left.=60: 40\right) ;[\alpha]^{29} \mathrm{D}+96.61\left(c 0.57, \mathrm{CH}_{2} \mathrm{Cl}_{2}\right) ;{ }^{1} \mathrm{H}$ NMR $\left(500 \mathrm{MHz}, \mathrm{CDCl}_{3}\right): \delta=7.33-$ $7.25(\mathrm{~m}, 5 \mathrm{H}), 6.20-6.17(\mathrm{dd}, J=5.35,10.03 \mathrm{~Hz}, 1 \mathrm{H}), 6.14-6.11(\mathrm{dd}, J=5.35,10.21 \mathrm{~Hz}, 1 \mathrm{H}), 5.97-5.94(\mathrm{dd}, J=$ $3.02,10.06 \mathrm{~Hz}, 1 \mathrm{H}), 5.92-5.80(\mathrm{~m}, 4 \mathrm{H}), 5.40-5.36(\mathrm{~d}, J=17.28 \mathrm{~Hz}, 1 \mathrm{H}), 5.32-5.16(\mathrm{~m}, 7 \mathrm{H}), 5.01-5.00(\mathrm{~d}, J=5.66$ $\mathrm{Hz}, 1 \mathrm{H}), 4.65-4.54(\mathrm{dd}, J=12.03,44.20 \mathrm{~Hz}, 2 \mathrm{H}), 4.16-4.13(\mathrm{dd}, J=6.70,6.70 \mathrm{~Hz}, 1 \mathrm{H}), 4.12-4.07(\mathrm{~m}, 2 \mathrm{H}), 4.01-$ 3.99 (dd, $J=3.99,7.42 \mathrm{~Hz}, 1 \mathrm{H}), 3.90-3 . .84(\mathrm{~m}, 1 \mathrm{H}), 3.79-3.74(\mathrm{qd}, J=6.54,6.54 \mathrm{~Hz}, 1 \mathrm{H}), 3.71-3.70$ (dd, $J=$ $2.45,5.43 \mathrm{~Hz}, 1 \mathrm{H}), 3.51-3.49$ (dd, $J=2.72,5.35 \mathrm{~Hz}, 1 \mathrm{H}), 2.09-2.08(\mathrm{~d}, J=4.09 \mathrm{~Hz}, 1 \mathrm{H}), 1.28-1.26(\mathrm{~d}, J=6.60$ $\mathrm{Hz}, 3 \mathrm{H}), 1.22-1.20(\mathrm{~d}, J=6.61 \mathrm{~Hz}, 3 \mathrm{H}), 1.15-1.13(\mathrm{~d}, J=6.43 \mathrm{~Hz}, 3 \mathrm{H}), 1.08-1.07(\mathrm{~d}, J=6.35 \mathrm{~Hz}, 3 \mathrm{H}) ;{ }^{13} \mathrm{C} \mathrm{NMR}$ $\left(125 \mathrm{MHz}, \mathrm{CDCl}_{3}\right): \delta=138.9,136.2,136.1,135.5,129.7,129.5,128.5,128.3,127.9,127.8,127.7,118.4,118.3$, 117.3, 102.7, 94.9, 94.4, 83.3, 81.5, 72.9, 70.9, 69.6, 69.2, 68.4, 66.7, 66.5, 18.1, 16.8, 16.1, 16.1; IR (NaCl): v 3482, 3081, 3031, 2980, 2895, 1453, 1427, 1404, 1379, 1341, 1313, 1254, $1193 \mathrm{~cm}^{-1}$; HRMS (ESI) m/z: [M+Na $]^{+}$ Calcd for $\mathrm{C}_{32} \mathrm{H}_{44} \mathrm{NaO}_{8}$ 579.2928; Found 579.2931.

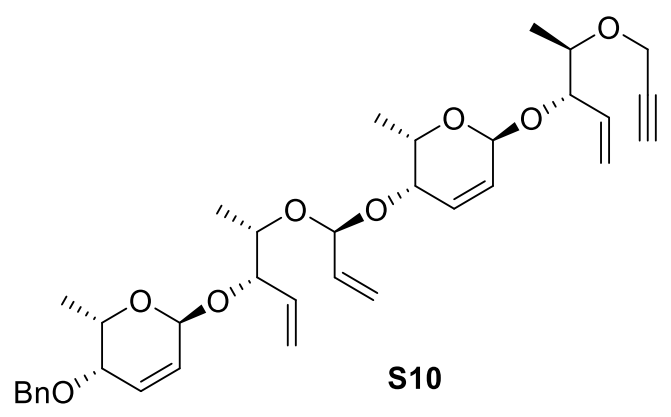

$(2 S, 3 S, 6 S)-3-($ benzyloxy)-2-methyl-6- $(((3 S, 4 S)-4-(((S))-1-(((2 S, 3 S, 6 S))-2-m e t h y l-6-(((3 S, 4 R)-4-(p r o p-2-y n-1-$ yloxy)pent-1-en-3-yl)oxy)-3,6-dihydro-2H-pyran-3-yl)oxy)allyl)oxy)pent-1-en-3-yl)oxy)-3,6-dihydro-2H-p yran (S10): To a suspension of $\mathrm{NaH}(96.0 \mathrm{mg}, 2.40 \mathrm{mmol}, 60 \%$ dispersion in mineral oil) in DMF was added S9 (441.4 mg, $0.80 \mathrm{mmol})$ in DMF $(4 \mathrm{ml})$ at $0{ }^{\circ} \mathrm{C}$ under nitrogen atmosphere. The reaction mixture was stirred for 10 $\mathrm{min}$ at room temperature. The solution of propargyl bromide $(0.3 \mathrm{ml}, 2.4 \mathrm{mmol}, 80 \% \mathrm{wt} \%$ in Toluene $)$ was added to a reaction mixture at $0{ }^{\circ} \mathrm{C}$. The resulting mixture was stirred for $2 \mathrm{~h}$ at room temperature. The reaction mixture was quenched with water $(30 \mathrm{ml})$, and then the aqueous phase was extracted with $\mathrm{Et}_{2} \mathrm{O}(4 \times 50 \mathrm{ml})$. The organic phase was washed with brine, dried over $\mathrm{Na}_{2} \mathrm{SO}_{4}$ and concentrated under reduced pressure. The crude product was purified by flash column chromatography (Hexane:Ethyl acetate $=90: 10$ ) to afford $\mathbf{S 1 0}$ as a colorless oil (457.9 mg, $0.77 \mathrm{mmol}, 96 \%)$.

$\mathrm{R}_{f} 0.50$ (Hexane:Ethyl acetate $\left.=80: 20\right) ;[\alpha]^{29} \mathrm{D}+112.55\left(c 0.98, \mathrm{CH}_{2} \mathrm{Cl}_{2}\right) ;{ }^{1} \mathrm{H} \mathrm{NMR}\left(500 \mathrm{MHz}, \mathrm{CDCl}_{3}\right): \delta=7.34-$ $7.25(\mathrm{~m}, 5 \mathrm{H}), 6.18-6.15(\mathrm{dd}, J=5.31,10.28 \mathrm{~Hz}, 1 \mathrm{H}), 6.14-6.11(\mathrm{dd}, J=5.14,10.28 \mathrm{~Hz}, 1 \mathrm{H}), 5.97-5.93(\mathrm{~m}, 2 \mathrm{H})$, 5.89-5.80 (m, 3H), 5.39-5.38 (d, $J=17.29,1 \mathrm{H}), 5.32-5.19(\mathrm{~m}, 7 \mathrm{H}), 5.01-5.00(\mathrm{~d}, J=5.69 \mathrm{~Hz}, 1 \mathrm{H}), 4.65-4.62(\mathrm{dd}$, $J=11.93,41.77 \mathrm{~Hz}, 2 \mathrm{H}), 4.27-4.18(\mathrm{~m}, 3 \mathrm{H}), 4.16-4.08(\mathrm{~m}, 3 \mathrm{H}), 3.79-3.75(\mathrm{~m}, 2 \mathrm{H}), 3.70-3.68(\mathrm{dd}, J=2.50,5.51$ $\mathrm{Hz}, 1 \mathrm{H}), 3.51-3.49(\mathrm{dd}, J=2.74,5.30 \mathrm{~Hz}, 1 \mathrm{H}), 2.39-2.38(\mathrm{t}, J=2.30 \mathrm{~Hz}, 1 \mathrm{H}), 1.28-1.26(\mathrm{~d}, J=6.76 \mathrm{~Hz}, 3 \mathrm{H})$, $1.21-1.20(\mathrm{~d}, J=6.62 \mathrm{~Hz}, 3 \mathrm{H}), 1.15-1.14(\mathrm{~d}, J=6.40 \mathrm{~Hz}, 3 \mathrm{H}), 1.08-1.07(\mathrm{~d}, J=6.40 \mathrm{~Hz}, 3 \mathrm{H}) ;{ }^{13} \mathrm{C}$ NMR $(125$ $\left.\mathrm{MHz}, \mathrm{CDCl}_{3}\right): \delta=138.9,136.3,136.1,136.0,129.7,129.2,128.6,128.5,127.9,127.8,127.7,118.3,117.22$, $117.0,102.7,94.9,94.4,81.5,80.5,80.3,76.5,74.2,72.8,70.9,69.6,68.6,66.7,66.3,56.6,16.8,16.3,16.1,15.2$; IR (NaCl): $v 3301,3263,3085,3040,2980,2933,2895,1450,1404,1379,1379,1343,1193 \mathrm{~cm}^{-1}$; HRMS (ESI) $\mathrm{m} / \mathrm{z}$ : $[\mathrm{M}+\mathrm{Na}]^{+}$Calcd for $\mathrm{C}_{35} \mathrm{H}_{46} \mathrm{NaO}_{8}$ 617.3085; Found 617.3089. 


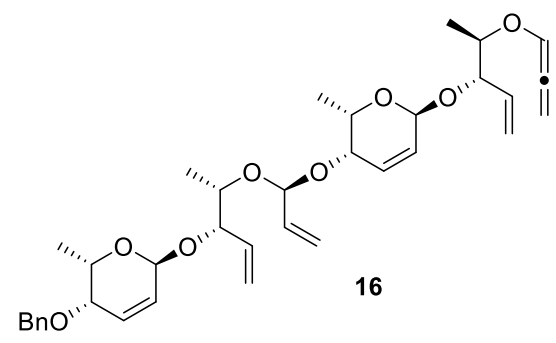

$(2 S, 3 S, 6 S)-3-(b e n z y l o x y)-2-m e t h y l-6-(((3 S, 4 S)-4-(((S)-1-(((2 S, 3 S, 6 S)-2-m e t h y l-6-(((3 S, 4 R)-4-(p r o p a-1,2-d i e$ n-1-yloxy)pent-1-en-3-yl)oxy)-3,6-dihydro-2H-pyran-3-yl)oxy)allyl)oxy)pent-1-en-3-yl)oxy)-3,6-dihydro-2 H-pyran (16): To a solution of $\mathbf{S 1 0}(594.7 \mathrm{mg}, 1.00 \mathrm{mmol})$ in THF (3.3 ml), was added tBuOK (56.1 $\mathrm{mg}, 0.50$ mmol) at $0{ }^{\circ} \mathrm{C}$ and stirred for $12 \mathrm{~h}$ at $0{ }^{\circ} \mathrm{C}$. The crude mixture was filtered with a pad of celite (rinse; $\mathrm{CH}_{2} \mathrm{Cl}_{2} 100$ $\mathrm{ml}$ ), then, concentrated under reduced pressure. The crude product was purified by flash column chromatography (Hexane:Ethyl acetate $=90: 10)$ to afford 16 as a colorless oil $(570.9 \mathrm{mg}, 0.96 \mathrm{mmol}, 96 \%)$.

$\mathrm{R}_{f} 0.54$ (Hexane:Ethyl acetate $\left.=80: 20\right) ;[\alpha]^{29} \mathrm{D}+142.68\left(c 0.58, \mathrm{CH}_{2} \mathrm{Cl}_{2}\right) ;{ }^{1} \mathrm{H} \mathrm{NMR}\left(500 \mathrm{MHz}, \mathrm{CDCl}_{3}\right): \delta=7.34-$ $7.26(\mathrm{~m}, 5 \mathrm{H}), 6.65-6.62(\mathrm{t}, J=5.99 \mathrm{~Hz}, 1 \mathrm{H}), 6.19-6.16(\mathrm{dd}, J=5.38,9.97 \mathrm{~Hz}, 1 \mathrm{H}), 6.14-6.11(\mathrm{dd}, J=5.27,9.97$ $\mathrm{Hz}, 1 \mathrm{H}), 5.97-5.94(\mathrm{~m}, 2 \mathrm{H}), 5.89-5.77(\mathrm{~m}, 3 \mathrm{H}), 5.42-5.36(\mathrm{~m}, 3 \mathrm{H}), 5.32-5.17(\mathrm{~m}, 7 \mathrm{H}), 5.02-5.01(\mathrm{~d}, J=5.53 \mathrm{~Hz}$, $1 \mathrm{H}), 4.65-4.54(\mathrm{dd}, J=11.86,42.10 \mathrm{~Hz}, 2 \mathrm{H}), 4.38-4.37(\mathrm{~m}, 1 \mathrm{H}), 4.16-4.07(\mathrm{~m}, 3 \mathrm{H}), 3.89-3.84(\mathrm{~m}, 1 \mathrm{H}), 3.80-3.75$ (qd, $J=6.34,6.34 \mathrm{~Hz}, 1 \mathrm{H}), 3.70-3.68(\mathrm{dd}, J=2.26,5.34 \mathrm{~Hz}, 1 \mathrm{H}), 3.51-3.50(\mathrm{dd}, J=2.60,5.06 \mathrm{~Hz}, 1 \mathrm{H}), 1.28-$ $1.27(\mathrm{~d}, J=6.74 \mathrm{~Hz}, 3 \mathrm{H}), 1.21-1.20(\mathrm{~d}, J=6.54 \mathrm{~Hz}, 3 \mathrm{H}), 1.19-1.17(\mathrm{~d}, J=6.27 \mathrm{~Hz}, 3 \mathrm{H}), 1.08-1.07(\mathrm{~d}, J=6.34$ $\mathrm{Hz}, 3 \mathrm{H}) ;{ }^{13} \mathrm{C} \mathrm{NMR}\left(125 \mathrm{MHz}, \mathrm{CDCl}_{3}\right): \delta=201.6,138.9,136.3,136.2,136.1,129.7,129.3,128.6,128.5,127.9$, $127.8,127.8,120.6,118.4,11.3,116.8,102.7,95.0,94.9,90.7,81.5,79.3,76.9,72.6,70.9,69.6,68.6,66.7,66.3$, 16.9, 16.3, 16.1, 14.5; IR (NaCl): v 3082, 3038, 2980, 2934, 2896, 1951, 1447, 1405, 1379, 1348, 1319, $1194 \mathrm{~cm}^{-}$ 1; HRMS (ESI) m/z: [M+Na] $]^{+}$Calcd for $\mathrm{C}_{35} \mathrm{H}_{46} \mathrm{NaO}_{8}$ 617.3085; Found 617.3087.

\section{Asymmetric addition of alcohol to allenes 16 via palladium catalyst and ring-closing metathesis}

General procedure of palladium-catalyzed asymmetric addition

A solution of $\mathrm{Pd}_{2}(\mathrm{dba})_{3}(2.5 \mathrm{~mol} \%)$ and $(S, S)$-L or $(R, R)-\mathrm{L}(5 \mathrm{~mol} \%)$ in toluene was added to an ene-allene (1 eq), alcohol $(2 \mathrm{eq})$ and triethylamine $(0.1 \mathrm{eq})$ in toluene (total concentration of solvent, $0.5 \mathrm{M})$ under nitrogen. The reaction mixture was stirred at $40{ }^{\circ} \mathrm{C}$ until TLC indicated complete conversion of the starting material. The crude product was purified by Flash column chromatography on silica gel to afford the desired product. Silica gel was deactivated with few drops of $\mathrm{Et}_{3} \mathrm{~N}$ and $\mathrm{CDCl}_{3}$ was deactivated with basic alumina before use.

\section{General procedure of $R C M$}

To a solution of acyclic $O, O$-acetal $(1 \mathrm{eq})$ dissolved in $\mathrm{CH}_{2} \mathrm{Cl}_{2}(0.1 \mathrm{M})$ was added the Grubbs catalyst. The reaction mixture was stirred at $40{ }^{\circ} \mathrm{C}$ until TLC indicated complete conversion of the starting material. The solvent was removed under reduced pressure and then the crude product was isolated by Flash column chromatography on silica gel. Silica gel was deactivated with few drops of $\mathrm{Et}_{3} \mathrm{~N}$ and $\mathrm{CDCl}_{3}$ was deactivated with basic alumina before use. 


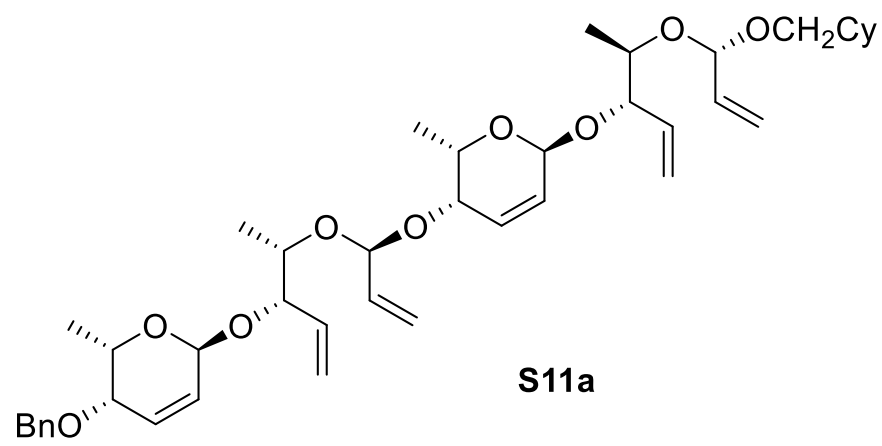

$(2 S, 3 S, 6 S)-3-($ benzyloxy $)-6-(((3 S, 4 S)-4-(((S))-1-(((2 S, 3 S, 6 S))-6-(((3 S, 4 R)-4-(((S))-1-(c y c l o h e x y l m e t h o x y) a l l y l))$ oxy)pent-1-en-3-yl)oxy)-2-methyl-3,6-dihydro-2H-pyran-3-yl)oxy)allyl)oxy)pent-1-en-3-yl)oxy)-2-methyl3,6-dihydro-2H-pyran (S11a): Following the general procedure, $\mathrm{CyCH}_{2} \mathrm{OH} 17(21.7 \mathrm{mg}, 0.190 \mathrm{mmol})$ was reacted with $\mathrm{Pd}_{2}(\mathrm{dba})_{3}(2.2 \mathrm{mg}, 0.002 \mathrm{mmol}),(R, R)-\mathrm{L}(3.6 \mathrm{mg}, 0.005 \mathrm{mmol})$, triethylamine $(1.5 \mu \mathrm{L}, 0.010 \mathrm{mmol})$ and ene-allene $16(56.5 \mathrm{mg}, 0.095 \mathrm{mmol})$ in toluene (total concentration of solvent, $0.5 \mathrm{M})$ under nitrogen. The reaction mixture was stirred for $3 \mathrm{~h}$ at $40{ }^{\circ} \mathrm{C}$. And then, concentrated under reduced pressure. the crude product was purified by flash column chromatography (Hexane:Ethyl acetate $=90: 10$ ) to afford S11a as a colorless oil. (62.6 $\mathrm{mg}, 0.088 \mathrm{mmol}, 93 \%$ yield). The diastereomer ratio (21:1) was determined by crude NMR.

$\mathrm{R}_{f} 0.57$ (Hexane:Ethyl acetate $\left.=80: 20\right) ;[\alpha]^{29} \mathrm{D}+91.03\left(c 0.81, \mathrm{CH}_{2} \mathrm{Cl}_{2}\right) ;{ }^{1} \mathrm{H}$ NMR $\left(500 \mathrm{MHz}, \mathrm{CDCl}_{3}\right): \delta=7.36-$ $7.26(\mathrm{~m}, 5 \mathrm{H}), 6.19-6.16(\mathrm{dd}, J=5.30,10.04 \mathrm{~Hz}, 1 \mathrm{H}), 6.15-6.11(\mathrm{dd}, J=5.17,10.19 \mathrm{~Hz}, 1 \mathrm{H}), 5.98-5.94(\mathrm{~m}, 2 \mathrm{H})$, $5.90-5.78(\mathrm{~m}, 4 \mathrm{H}), 5.40-5.16(\mathrm{~m}, 10 \mathrm{H}), 5.03-5.02(\mathrm{~d}, J=5.62 \mathrm{~Hz}, 1 \mathrm{H}), 4.97-4.96(\mathrm{td}, J=0.92,4.86 \mathrm{~Hz}, 1 \mathrm{H})$, 4.65-4.63 (dd, $J=11.99,42.41 \mathrm{~Hz}, 2 \mathrm{H}), 4.28-4.26(\mathrm{~m}, 1 \mathrm{H}), 4.16-4.08(\mathrm{~m}, 3 \mathrm{H}), 3.87-3.82(\mathrm{~m}, 1 \mathrm{H}), 3.80-3.75(\mathrm{qd}$, $J=6.48,6.48 \mathrm{~Hz}, 1 \mathrm{H}), 3.71-3.70(\mathrm{dd}, J=2.39,5.42 \mathrm{~Hz}, 1 \mathrm{H}), 3.52-3.50(\mathrm{dd}, J=2.64,5.19 \mathrm{~Hz}, 1 \mathrm{H}), 3.37-3.22(\mathrm{~m}$, 2H), 1.76-1.62 (m, 5H), 1.29-1.08 (m, 15H), 0.97-0.88 (m, 2H); ${ }^{13} \mathrm{C}$ NMR (125 MHz, $\left.\mathrm{CDCl}_{3}\right): \delta=138.9,136.8$, $136.3,136.2,136.1,129.7,129.1,128.7,128.5,127.9,127.8,127.7,118.4,118.2,117.3,116.5,102.7,100.2,94.9$, $94.8,81.5,81.5,73.3,72.7,70.9,70.9,69.6,68.8,66.7,66.3,38.4,30.4,30.4,26.8,26.1,16.9,16.3,16.1,15.0$; IR (NaCl): v 3086, 3029, 2981, 2925, 2854, 1449, 1428, 1406, 1379, 1342, 1320, $1192 \mathrm{~cm}^{-1}$; HRMS (ESI) m/z: $[\mathrm{M}+\mathrm{Na}]^{+}$Calcd for $\mathrm{C}_{42} \mathrm{H}_{60} \mathrm{NaO}_{9} 731.4130$; Found 731.4132 .

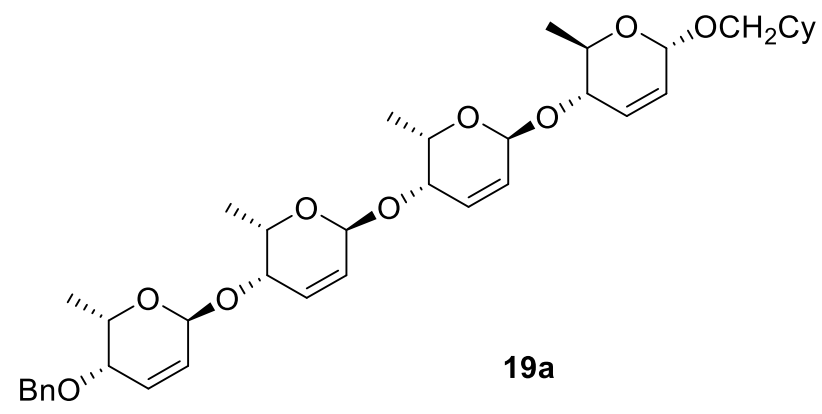

$(2 S, 3 S, 6 S)-3-($ benzyloxy)-6- (( (2S,3S,6S)-6-(((2S,3S,6S)-6-(((2R,3S,6S)-6-(cyclohexylmethoxy)-2-methyl-3,6dihydro-2H-pyran-3-yl)oxy)-2-methyl-3,6-dihydro-2H-pyran-3-yl)oxy)-2-methyl-3,6-dihydro-2H-pyran-3yl)oxy)-2-methyl-3,6-dihydro-2H-pyran (19a): Following the general procedure, the O,O-acetal compound S11a (34.0 mg, $0.048 \mathrm{mmol})$ was reacted with catalyst GII $(12.3 \mathrm{mg}, 0.014 \mathrm{mmol})$ in $\mathrm{CH}_{2} \mathrm{Cl}_{2}(0.96 \mathrm{ml})$. The reaction mixture was stirred for $22 \mathrm{~h}$ at $40{ }^{\circ} \mathrm{C}$. And then, concentrated under reduced pressure. the crude product was purified by flash column chromatography (Hexane:Ethyl acetate $=80: 20$ ) to afford 19a as a white solid (28.7 $\mathrm{mg}, 0.044 \mathrm{mmol}, 92 \%$ yield).

m.p. 186-188 ${ }^{\circ} \mathrm{C} ; \mathrm{R}_{f} 0.33$ (Hexane:Ethyl acetate $\left.=80: 20\right) ;[\alpha]^{29}{ }_{\mathrm{D}}+322.70\left(c 0.42, \mathrm{CH}_{2} \mathrm{Cl}_{2}\right) ;{ }^{1} \mathrm{H}$ NMR $(500 \mathrm{MHz}$, $\left.\mathrm{CDCl}_{3}\right): \delta=7.34-7.26(\mathrm{~m}, 5 \mathrm{H}), 6.15-6.09(\mathrm{~m}, 3 \mathrm{H}), 6.03-5.97(\mathrm{~m}, 2 \mathrm{H}), 5.94-5.91(\mathrm{dd}, J=2.98,9.98 \mathrm{~Hz}, 1 \mathrm{H}), 5.89-$ 
$5.86(\mathrm{dd}, J=3.05,9.98 \mathrm{~Hz}, 1 \mathrm{H}), 5.73-5.70(\mathrm{~m}, 1 \mathrm{H}), 5.11-5.10(\mathrm{~d}, J=2.87 \mathrm{~Hz}, 1 \mathrm{H}), 5.07-5.06(\mathrm{~d}, J=2.73 \mathrm{~Hz}$, $1 \mathrm{H}), 5.04-5.03(\mathrm{~d}, J=2.85 \mathrm{~Hz}, 1 \mathrm{H}), 4.88-4.87(\mathrm{~m}, 1 \mathrm{H}), 4.65-4.54(\mathrm{dd}, J=11.95,411.42 \mathrm{~Hz}, 2 \mathrm{H}), 4.20-4.13(\mathrm{~m}$, $3 \mathrm{H}), 3.91-3.84(\mathrm{~m}, 2 \mathrm{H}), 3.65-3.63(\mathrm{dd}, J=2.33,5.49 \mathrm{~Hz}, 2 \mathrm{H}), 3.54-3.51(\mathrm{dd}, J=7.00,9.35 \mathrm{~Hz}, 1 \mathrm{H}), 3.49-3.47$ (dd, $J=2.62,5.22 \mathrm{~Hz}, 1 \mathrm{H}), 3.28-3.25(\mathrm{dd}, J=6.19,9.37 \mathrm{~Hz}, 1 \mathrm{H}), 1.79-1.54(\mathrm{~m}, 6 \mathrm{H}), 1.34-1.33(\mathrm{~d}, J=6.70 \mathrm{~Hz}$, $3 \mathrm{H}), 1.29-1.14$ (m, 12H), 0.94-0.90 (m, 2H); ${ }^{13} \mathrm{C}$ NMR (125 MHz, $\left.\mathrm{CDCl}_{3}\right): \delta=138.8$ 132.7, 129.7, 129.7, 129.0, 128.6, 128.0, 127.9, 127.8, 127.7, 126.9, 96.4, 96.3, 96.3, 94.7, 77.6, 74.4, 71.0, 70.4, 70.4, 67.6, 66.6, 66.0, 65.9, $38.3,30.3,30.2,26.8,26.1,26.0,18.3,16.6,16.4,16.3$; IR (NaCl): v 3042, 2979, 2918, 2853, 1448, 1401, 1379 , 1311, 1192, 1154, 1128, $1099 \mathrm{~cm}^{-1}$; HRMS (ESI) m/z: [M+Na] $]^{+}$Calcd for $\mathrm{C}_{38} \mathrm{H}_{52} \mathrm{NaO}_{9}$ 675.3504; Found 675.3506.

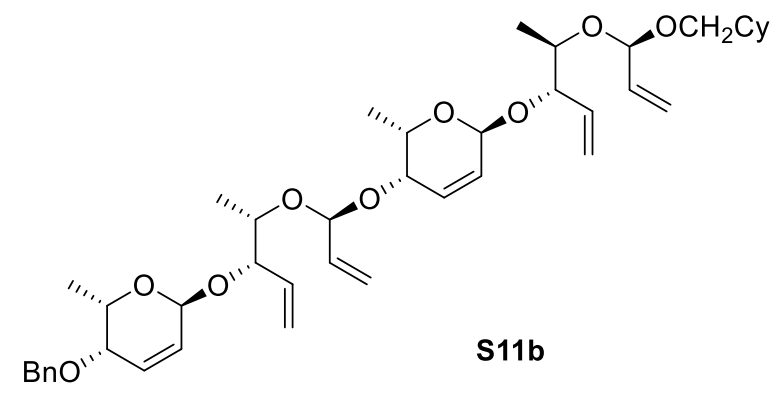

$(2 S, 3 S, 6 S)-3-($ benzyloxy)-6- $(((3 S, 4 S)-4-(((S)-1-)((2 S, 3 S, 6 S)-6-(((3 S, 4 R)-4-(((R))-1-($ cyclohexylmethoxy)ally l)oxy)pent-1-en-3-yl)oxy)-2-methyl-3,6-dihydro-2H-pyran-3-yl)oxy)allyl)oxy)pent-1-en-3-yl)oxy)-2-methyl -3,6-dihydro-2H-pyran (S11b): Following the general procedure, $\mathrm{CyCH}_{2} \mathrm{OH} 17$ (21.7 mg, $0.190 \mathrm{mmol}$ ) was reacted with $\mathrm{Pd}_{2}(\mathrm{dba})_{3}(2.2 \mathrm{mg}, 0.002 \mathrm{mmol}),(S, S)-\mathrm{L}(3.6 \mathrm{mg}, 0.005 \mathrm{mmol})$, triethylamine $(1.5 \mu \mathrm{L}, 0.010 \mathrm{mmol})$ and ene-allene $16(56.5 \mathrm{mg}, 0.095 \mathrm{mmol})$ in toluene (total concentration of solvent, $0.5 \mathrm{M})$ under nitrogen. The reaction mixture was stirred for $3 \mathrm{~h}$ at $40{ }^{\circ} \mathrm{C}$. And then, concentrated under reduced pressure. the crude product was purified by flash column chromatography (Hexane:Ethyl acetate $=90: 10$ ) to afford S11b as a colorless oil ( $60.0 \mathrm{mg}, 0.084 \mathrm{mmol}, 89 \%$ yield). The diastereomer ratio (1:17) was determined by crude NMR.

$\mathrm{R}_{f} 0.57$ (Hexane:Ethyl acetate $\left.=80: 20\right) ;[\alpha]^{29} \mathrm{D}+112.80\left(c 0.24, \mathrm{CH}_{2} \mathrm{Cl}_{2}\right) ;{ }^{1} \mathrm{H} \mathrm{NMR}\left(500 \mathrm{MHz}, \mathrm{CDCl}_{3}\right): \delta=7.35-$ $7.26(\mathrm{~m}, 5 \mathrm{H}), 6.18-6.15(\mathrm{dd}, J=5.38,10.01 \mathrm{~Hz}, 1 \mathrm{H}), 6.14-6.11(\mathrm{dd}, J=5.09,10.01 \mathrm{~Hz}, 1 \mathrm{H}), 5.97-5.92(\mathrm{~m}, 2 \mathrm{H})$, 5.89-5.76 (m, 4H), 5.40-5.16 (m, 10H), 5.02-5.00 (d, $J=5.34 \mathrm{~Hz}, 1 \mathrm{H}), 4.65-4.54(\mathrm{dd}, J=11.85,42.36 \mathrm{~Hz}, 2 \mathrm{H})$, 4.26-4.20 (m, 1H), 4.15-4.07 (m, 3H), 3.86-3.81 (m, $1 \mathrm{H}), 3.79-3.74(\mathrm{qd}, J=6.34,6.34 \mathrm{H}, 1 \mathrm{H}), 3.70-3.68(\mathrm{dd}, J=$ 2.37, $5.37 \mathrm{~Hz}, 1 \mathrm{H}), 3.51-3.50(\mathrm{dd}, J=2.80,5.30 \mathrm{~Hz}, 1 \mathrm{H}), 3.33-3.21(\mathrm{~m}, 2 \mathrm{H}), 1.76-1.64(\mathrm{~m}, 5 \mathrm{H}), 1.56-1.51(\mathrm{~m}$, $1 \mathrm{H}), 1.28-1.07(\mathrm{~m}, 15 \mathrm{H}), 0.97-0.86(\mathrm{~m}, 2 \mathrm{H}) ;{ }^{13} \mathrm{C}$ NMR $\left(125 \mathrm{MHz}, \mathrm{CDCl}_{3}\right): \delta=138.9,136.6,136.3,136.1,135.8$, 129.7, 129.2, 128.7, 128.6, 127.9, 127.8, 127.8, 118.4, 118.1, 117.3, 116.7, 102.7, 101.6, 94.9, 94.8, 81.5, 81.0, $74.7,72.7,70.9,70.7,69.6,68.7,66.7,66.3,38.4,30.4,26.8,26.1,16.9,16.4,16.3,16.1 ; \mathrm{IR}(\mathrm{NaCl}): v 3081,3029$, 2980, 2924, 2854, 1451, 1428, 1406, 1378, 1321, 1192, $1159 \mathrm{~cm}^{-1}$; HRMS (ESI) m/z: $[\mathrm{M}+\mathrm{Na}]^{+}$Calcd for $\mathrm{C}_{42} \mathrm{H}_{60} \mathrm{NaO}_{9} 731.4130$; Found 731.4127

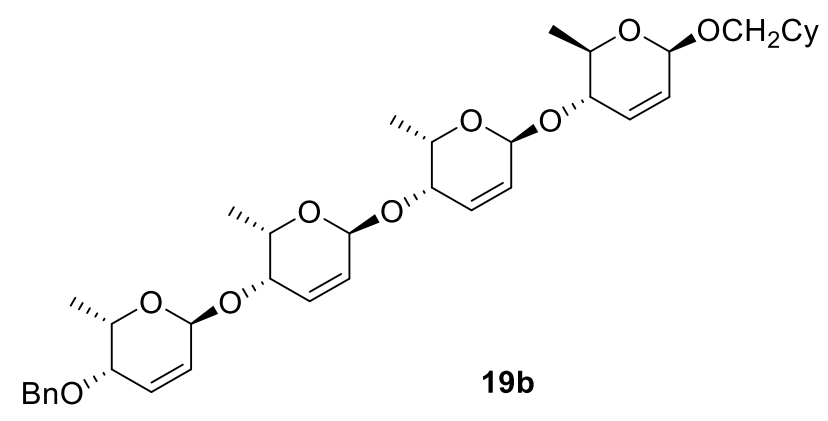




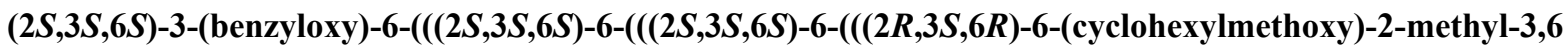
-dihydro-2H-pyran-3-yl)oxy)-2-methyl-3,6-dihydro-2H-pyran-3-yl)oxy)-2-methyl-3,6-dihydro-2H-pyran-3 -yl)oxy)-2-methyl-3,6-dihydro-2H-pyran (19b): Following the general procedure, the $\mathrm{O}, \mathrm{O}$-acetal compound S11b $(15.6 \mathrm{mg}, 0.022 \mathrm{mmol})$ was reacted with catalyst GII $(5.6 \mathrm{mg}, 0.0066 \mathrm{mmol})$ in $\mathrm{CH}_{2} \mathrm{Cl}_{2}(0.44 \mathrm{ml})$. The reaction mixture was stirred for $11 \mathrm{~h}$ at $40{ }^{\circ} \mathrm{C}$. And then, concentrated under reduced pressure. the crude product was purified by flash column chromatography (Hexane:Ethyl acetate $=80: 20$ ) to afford $\mathbf{1 9 b}$ as a white solid (13.2 $\mathrm{mg}, 0.020 \mathrm{mmol}, 91 \%$ yield).

m.p. 186- $188{ }^{\circ} \mathrm{C} ; \mathrm{R}_{f} 0.31$ (Hexane:Ethyl acetate $\left.=70: 30\right) ;[\alpha]^{29} \mathrm{D}+277.30\left(c 0.81, \mathrm{CH}_{2} \mathrm{Cl}_{2}\right) ;{ }^{1} \mathrm{H} \mathrm{NMR}(500 \mathrm{MHz}$, $\left.\mathrm{CDCl}_{3}\right): \delta=7.34-7.26(\mathrm{~m}, 5 \mathrm{H}), 6.14-6.11(\mathrm{~m}, 3 \mathrm{H}), 6.03-6.02(\mathrm{dd}, J=2.96,10.07 \mathrm{~Hz}, 1 \mathrm{H}), 5.96-5.97(\mathrm{~m}, 2 \mathrm{H})$, 5.88-5.86 (dd, $J=2.98,10.07 \mathrm{~Hz}, 1 \mathrm{H}), 5.77-5.75(\mathrm{td}, J=1.52,10.36 \mathrm{~Hz}, 1 \mathrm{H}), 5.11-5.03(\mathrm{~m}, 4 \mathrm{H}), 4.65-4.54(\mathrm{dd}$, $J=11.93,41.50 \mathrm{~Hz}, 2 \mathrm{H}), 4.19-4.13(\mathrm{~m}, 3 \mathrm{H}), 3.94-3.92(\mathrm{~m}, 1 \mathrm{H}), 3.74-3.69$ (qd, $J=6.28,6.28 \mathrm{~Hz}, 1 \mathrm{H}), 3.65-3.61$ $(\mathrm{m}, 3 \mathrm{H}), 3.49-3.47(\mathrm{dd}, J=2.73,5.35 \mathrm{~Hz}, 1 \mathrm{H}), 3.28-3.24(\mathrm{~m}, 1 \mathrm{H}), 1.78-1.53(\mathrm{~m}, 6 \mathrm{H}), 1.34-1.13(\mathrm{~m}, 15 \mathrm{H}), 0.96-$ $0.87(\mathrm{~m}, 2 \mathrm{H}) ;{ }^{13} \mathrm{C}$ NMR $\left(125 \mathrm{MHz}, \mathrm{CDCl}_{3}\right): \delta=138.8,131.6,129.8,129.7,129.6,129.0,128.5,128.0,127.9$, $127.8,127.7,97.2,96.3,96.3,95.7,77.4,76.1,73.9,72.5,71.1,70.4,70.4,69.6,66.6,66.0,65.9,38.3,36.3,36.2$, 26.8, 26.1, 18.7, 16.6, 16.4, 16.3; IR (NaCl): $\vee 3045,2976,2921,2852,1452,1401,1380,1193,1156,1098,1065$, $964 \mathrm{~cm}^{-1}$; HRMS (ESI) m/z: [M+Na] Calcd for $\mathrm{C}_{38} \mathrm{H}_{52} \mathrm{NaO}_{9}$ 675.3504; Found 675.3505.

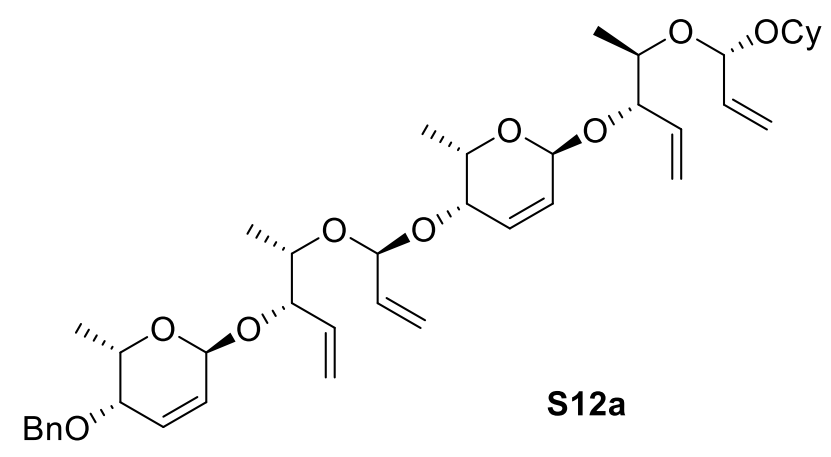

(2S,3S,6S)-3-(benzyloxy)-6- $(((3 S, 4 S))-4-(((S))-1-(((2 S, 3 S, 6 S)-6-(((3 S, 4 R)-4-(((S))-1-($ cyclohexyloxy)allyl)oxy)p ent-1-en-3-yl)oxy)-2-methyl-3,6-dihydro-2H-pyran-3-yl)oxy)allyl)oxy)pent-1-en-3-yl)oxy)-2-methyl-3,6-di hydro-2H-pyran (S12a): Following the general procedure, $\mathrm{CyOH} 18(20.1 \mathrm{mg}, 0.200 \mathrm{mmol})$ was reacted with $\mathrm{Pd}_{2}(\mathrm{dba})_{3}(9.2 \mathrm{mg}, 0.010 \mathrm{mmol}),(R, R)-\mathrm{L}(18.9 \mathrm{mg}, 0.020 \mathrm{mmol})$, triethylamine $(1.5 \mu \mathrm{L}, 0.010 \mathrm{mmol})$ and eneallene $16(59.5 \mathrm{mg}, 0.100 \mathrm{mmol})$ in toluene (total concentration of solvent, $0.5 \mathrm{M})$ under nitrogen. The reaction mixture was stirred for $6 \mathrm{~h}$ at $40^{\circ} \mathrm{C}$. And then, concentrated under reduced pressure. the crude product was purified by flash column chromatography (Hexane:Ethyl acetate $=90: 10)$ to afford S12a as a colorless oil (62.5 mg, 0.090 mmol, $90 \%$ yield, diastereomeric ratio was not determined).

$\mathrm{R}_{f} 0.56$ (Hexane:Ethyl acetate $\left.=80: 20\right) ;[\alpha]^{29} \mathrm{D}+119.42\left(c 0.39, \mathrm{CH}_{2} \mathrm{Cl}_{2}\right) ;{ }^{1} \mathrm{H} \mathrm{NMR}\left(500 \mathrm{MHz}, \mathrm{CDCl}_{3}\right): \delta=7.33-$ $7.26(\mathrm{~m}, 5 \mathrm{H}), 6.18-6.14(\mathrm{dd}, J=5.32,10.01 \mathrm{~Hz}, 1 \mathrm{H}), 6.14-6.10(\mathrm{dd}, J=5.24,10.01 \mathrm{~Hz}, 1 \mathrm{H}), 5.97-5.93(\mathrm{~m}, 2 \mathrm{H})$, 5.89-5.80 (m, 4H), 5.39-5.16 (m, 10H), 5.03-5.01 (m, 2H), 4.65-4.54 (dd, $J=11.93,42.06 \mathrm{~Hz}, 2 \mathrm{H}), 4.23-4.22(\mathrm{~m}$, $1 \mathrm{H}), 4.15-4.07(\mathrm{~m}, 3 \mathrm{H}), 3.84-3.74(\mathrm{~m}, 2 \mathrm{H}), 3.70-3.68(\mathrm{dd}, J=2.29,5.38 \mathrm{~Hz}, 1 \mathrm{H}), 3.63-3.57(\mathrm{~m}, 1 \mathrm{H}), 3.51-3.49$ $(\mathrm{dd}, J=2.61,5.14 \mathrm{~Hz}, 1 \mathrm{H}), 1.86-1.85(\mathrm{~m}, 2 \mathrm{H}), 1.73-1.71(\mathrm{~m}, 2 \mathrm{H}), 1.52-1.50(\mathrm{~m}, 1 \mathrm{H}), 1.40-1.07(\mathrm{~m}, 17 \mathrm{H}) ;{ }^{13} \mathrm{C}$ $\operatorname{NMR}\left(125 \mathrm{MHz}, \mathrm{CDCl}_{3}\right): \delta=138.9,137.0,136.8,136.4,136.2,129.7,129.1,128.8,128.5,127.9,127.8,127.8$, $118.4,117.6,117.3,116.5,102.7,99.4,94.9,94.8,81.8,81.5,73.9,73.0,72.7,70.9,69.7,68.8,66.7,66.3,33.5$, 32.7, 25.9, 24.5, 24.3, 16.9, 16.3, 16.1, 15.7; IR (NaCl): v 3081, 3031, 2980, 2933, 2858, 1453, 1428, 1405, 1378, 1342, 1317, $1193 \mathrm{~cm}^{-1}$; HRMS (ESI) m/z: [M+Na] ${ }^{+}$Calcd for $\mathrm{C}_{41} \mathrm{H}_{58} \mathrm{NaO}_{9}$ 717.3973; Found 717.3973. 


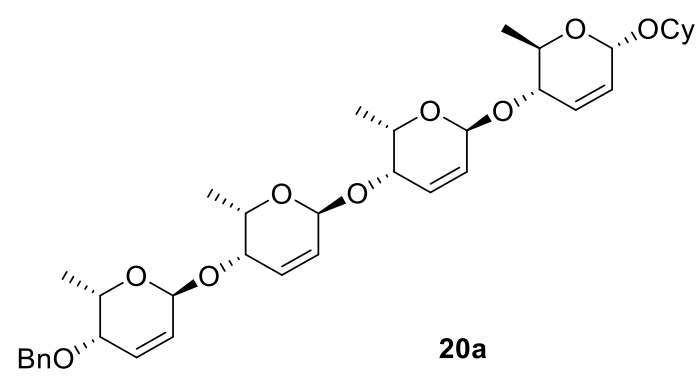

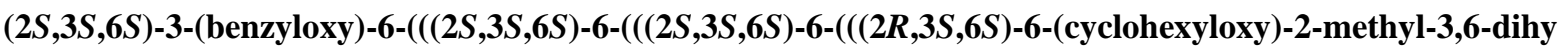
dro-2H-pyran-3-yl)oxy)-2-methyl-3,6-dihydro-2H-pyran-3-yl)oxy)-2-methyl-3,6-dihydro-2H-pyran-3-yl)o xy)-2-methyl-3,6-dihydro-2H-pyran (20a): Following the general procedure, the O,O-acetal compound S12a $(47.9 \mathrm{mg}, 0.069 \mathrm{mmol})$ was reacted with catalyst $\mathbf{G I I}(12.3 \mathrm{mg}, 0.069 \mathrm{mmol})$ in $\mathrm{CH}_{2} \mathrm{Cl}_{2}(0.96 \mathrm{ml})$. The reaction mixture was stirred for $15 \mathrm{~h}$ at $40{ }^{\circ} \mathrm{C}$. And then, concentrated under reduced pressure. the crude product was purified by flash column chromatography (Hexane:Ethyl acetate $=80: 20$ ) to afford 20a as a white solid $(37.7 \mathrm{mg}$, $0.059 \mathrm{mmol}, 86 \%$ yield). The diastereomer ratio (15:1) was determined by crude NMR.

m.p. 187-189 ${ }^{\circ} \mathrm{C} ; \mathrm{R}_{f} 0.34$ (Hexane:Ethyl acetate $\left.=80: 20\right) ;[\alpha]^{29} \mathrm{D}+331.27\left(c 0.52, \mathrm{CH}_{2} \mathrm{Cl}_{2}\right) ;{ }^{1} \mathrm{H} \mathrm{NMR}(500 \mathrm{MHz}$, $\left.\mathrm{CDCl}_{3}\right): \delta=7.34-7.26(\mathrm{~m}, 5 \mathrm{H}), 6.15-6.09(\mathrm{~m}, 3 \mathrm{H}), 6.03-5.97(\mathrm{~m}, 2 \mathrm{H}), 5.94-5.91(\mathrm{dd}, J=3.02,10.02 \mathrm{~Hz}, 1 \mathrm{H})$, 5.89-5.86 (dd, $J=3.05,10.02 \mathrm{~Hz}, 1 \mathrm{H}), 5.71-5.68(\mathrm{td}, J=2.19,10.26 \mathrm{~Hz}, 1 \mathrm{H}), 5.11-5.110(\mathrm{~d}, J=2.86 \mathrm{~Hz}, 1 \mathrm{H})$, 5.07-5.05 (m, 2H), 5.04-5.03 (d, $J=2.82 \mathrm{~Hz}, 1 \mathrm{H}), 4.65-4.54(\mathrm{dd}, J=11.96,41.57 \mathrm{~Hz}, 2 \mathrm{H}), 4.20-4.13(\mathrm{~m}, 3 \mathrm{H})$, 3.96-3.91 (m, 1H), 3.86-3.84 (m, 1H), 3.65-3.57 (m, 3H), 3.49-3.47 (dd, $J=2.61,5.19 \mathrm{~Hz}, 1 \mathrm{H}), 1.87-1.86(\mathrm{~m}$, $2 \mathrm{H}), 1.73-1.71(\mathrm{~m}, 2 \mathrm{H}), 1.52-1.51(\mathrm{~m}, 1 \mathrm{H}), 1.41-1.16(\mathrm{~m}, 17 \mathrm{H}) ;{ }^{13} \mathrm{C} \mathrm{NMR}\left(125 \mathrm{MHz}, \mathrm{CDCl}_{3}\right): \delta=138.8,132.6$, $129.7,129.7,129 . .0,128.6,128.1,127.9,127.8,127.7,127.5,96.4,96.3,96.3,92.8,77.8,76.2,71.0,70.5,70.4$, 69.6, 66.6, 65.9, 65.9, 66.8, 34.2, 32.4, 25.9, 24.6 24.4, 18.3, 16.6, 16.4 16.3; IR (NaCl): v 3042, 29.82, 2929, 2857, 1451, 1399, 1380, 1303, 1192, 1154, 1128, $1099 \mathrm{~cm}^{-1}$; HRMS (ESI) m/z: $[\mathrm{M}+\mathrm{Na}]^{+}$Calcd for $\mathrm{C}_{37} \mathrm{H}_{50} \mathrm{NaO}_{9}$ 661.3347; Found 661.3348.

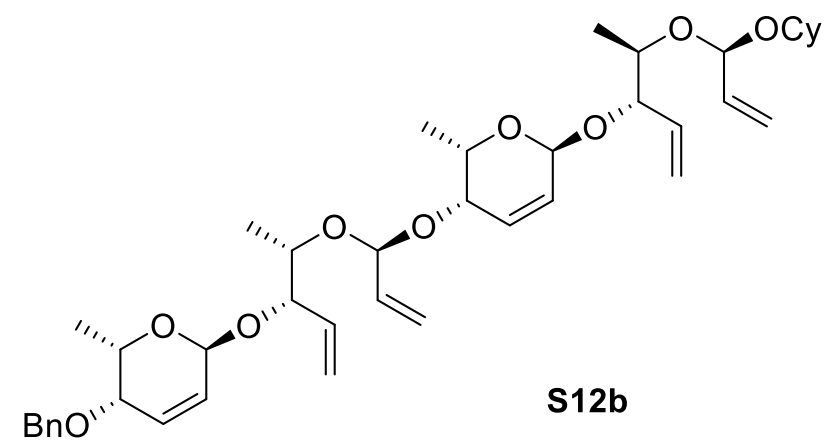

(2S,3S,6S)-3-(benzyloxy)-6-(((3S,4S)-4- $(((S))-1-(((2 S, 3 S, 6 S)-6-(((3 S, 4 R)-4-(((R))-1-($ cyclohexyloxy $)$ allyl)oxy $) p$ ent-1-en-3-yl)oxy)-2-methyl-3,6-dihydro-2H-pyran-3-yl)oxy)allyl)oxy)pent-1-en-3-yl)oxy)-2-methyl-3,6-di hydro-2H-pyran (S12b): Following the general procedure, CyOH 18 (40.2 mg, $0.400 \mathrm{mmol})$ was reacted with $\mathrm{Pd}_{2}(\mathrm{dba})_{3}(18.3 \mathrm{mg}, 0.020 \mathrm{mmol}),(S, S)-\mathrm{L}(27.6 \mathrm{mg}, 0.040 \mathrm{mmol})$, triethylamine $(3 \mu \mathrm{L}, 0.020 \mathrm{mmol})$ and eneallene $16(118.9 \mathrm{mg}, 0.200 \mathrm{mmol})$ in toluene (total concentration of solvent, $0.5 \mathrm{M})$ under nitrogen. The reaction mixture was stirred for $8 \mathrm{~h}$ at $40^{\circ} \mathrm{C}$. And then, concentrated under reduced pressure. the crude product was purified by flash column chromatography (Hexane:Ethyl acetate $=90: 10)$ to afford $\mathbf{S 1 2 b}$ as a colorless oil. $(83.4 \mathrm{mg}, 0.120$ $\mathrm{mmol}, 60 \%$ yield, diastereomeric ratio was not determined).

$\mathrm{R}_{f} 0.57$ (Hexane:Ethyl acetate $\left.=80: 20\right) ;[\alpha]^{29} \mathrm{D}+106.67\left(c 0.52, \mathrm{CH}_{2} \mathrm{Cl}_{2}\right) ;{ }^{1} \mathrm{H}$ NMR $\left(500 \mathrm{MHz}, \mathrm{CDCl}_{3}\right): \delta=7.34-$ $7.26(\mathrm{~m}, 5 \mathrm{H}), 6.18-6.15(\mathrm{dd}, J=5.42,10.10 \mathrm{~Hz}, 1 \mathrm{H}), 6.14-6.10(\mathrm{dd}, J=5.20,10.10 \mathrm{~Hz}, 1 \mathrm{H}), 5.97-5.92(\mathrm{~m}, 2 \mathrm{H})$, 6.89-5.79 (m, 4H), 5.39-5.15 (m, 10H), 5.06-5.05 (d, $J=5.28 \mathrm{~Hz}, 1 \mathrm{H}), 5.02-5.00(\mathrm{~d}, J=5.62 \mathrm{~Hz}, 1 \mathrm{H}), 4.65-4 . .54$ 
(dd, $J=11.99,42.08 \mathrm{~Hz}, 2 \mathrm{H}), 4.23-4.21(\mathrm{~m}, 1 \mathrm{H}), 4.15-4.07(\mathrm{~m}, 3 \mathrm{H}), 3.82-3.75(\mathrm{~m}, 2 \mathrm{H}), 3.70-3.68(\mathrm{dd}, J=2.36$, $5.38 \mathrm{~Hz}, 1 \mathrm{H}), 3.55-3.49(\mathrm{~m}, 2 \mathrm{H}), 1.87-1.81(\mathrm{~m}, 2 \mathrm{H}), 1.74-1.71(\mathrm{~m}, 2 \mathrm{H}), 1.53-1.51(\mathrm{~m}, 1 \mathrm{H}), 1.37-1.07(\mathrm{~m}, 17 \mathrm{H})$; ${ }^{13} \mathrm{C}$ NMR $\left(125 \mathrm{MHz}, \mathrm{CDCl}_{3}\right): \delta=138.9,136.8,136.7,136.3,136.1,129.7,129.1,128.7,128.5,127.9,127.8$, $127.8,118.4,117.5,117.3,116.6,102.7,100.3,94.9,94.9,81.5,81.0,74.4,73.9,72.7,700.9,69.7,68.7,66.7$, $66.3,33.4,33.1,25.9,24.5,24.5,16.8,16.3,16.3,16.1$; IR (NaCl): $v 3086,3029,2981,2933,2858,1450,1406$, 1378, 1319, 1193, 1159, $1126 \mathrm{~cm}^{-1}$; HRMS (ESI) m/z: [M+Na] Calcd for $\mathrm{C}_{41} \mathrm{H}_{58} \mathrm{NaO}_{9}$ 717.3973; Found 717.3974.

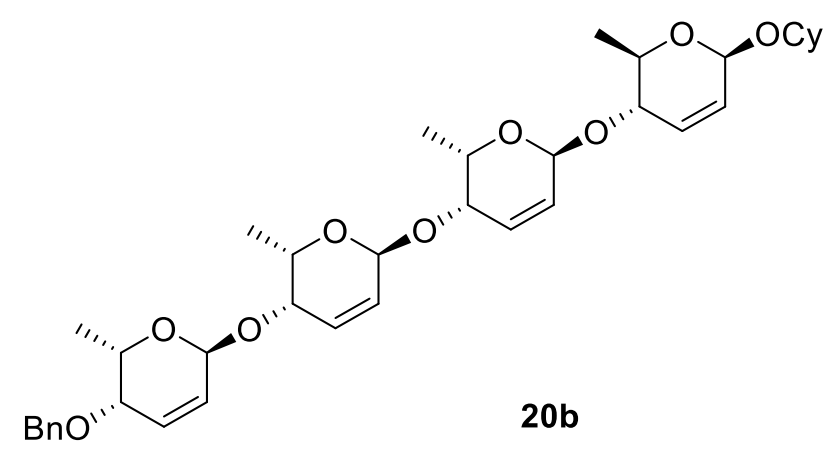

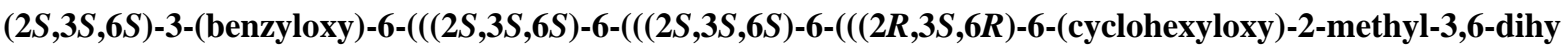
dro-2H-pyran-3-yl)oxy)-2-methyl-3,6-dihydro-2H-pyran-3-yl)oxy)-2-methyl-3,6-dihydro-2H-pyran-3-yl)o xy)-2-methyl-3,6-dihydro-2H-pyran (20b): Following the general procedure, the O,O-acetal compound S12b $(62.5 \mathrm{mg}, 0.090 \mathrm{mmol})$ was reacted with catalyst $\mathbf{G I I}(22.9 \mathrm{mg}, 0.027 \mathrm{mmol})$ in $\mathrm{CH}_{2} \mathrm{Cl}_{2}(1.8 \mathrm{ml})$. The reaction mixture was stirred for $16 \mathrm{~h}$ at $40{ }^{\circ} \mathrm{C}$. And then, concentrated under reduced pressure. the crude product was purified by flash column chromatography (Hexane:Ethyl acetate $=80: 20$ ) to afford $20 \mathrm{~b}$ as a white solid. $(52.3 \mathrm{mg}$, $0.082 \mathrm{mmol}, 90 \%$ yield). The diastereomer ratio (1:13) was determined by crude NMR.

m.p. 187-189 ${ }^{\circ} \mathrm{C} ; \mathrm{R}_{f} 0.33$ (Hexane:Ethyl acetate $\left.=80: 20\right) ;[\alpha]^{29}{ }_{\mathrm{D}}+335.61\left(c 0.60, \mathrm{CH}_{2} \mathrm{Cl}_{2}\right) ;{ }^{1} \mathrm{H} \mathrm{NMR}(500 \mathrm{MHz}$, $\left.\mathrm{CDCl}_{3}\right): \delta=7.34-7.26(\mathrm{~m}, 5 \mathrm{H}), 6.14-6.09(\mathrm{~m}, 3 \mathrm{H}) 6.03-6.00(\mathrm{dd}, J=2.92,10.14 \mathrm{~Hz}, 1 \mathrm{H}), 5.95-5.91(\mathrm{~m}, 2 \mathrm{H})$, $5.88-5.85(\mathrm{dd}, J=2.94,9.95 \mathrm{~Hz}, 1 \mathrm{H}), 5.21-5.20(\mathrm{~m}, 1 \mathrm{H}), 5.11-5.10(\mathrm{~d}, J=2.73 \mathrm{~Hz}, 1 \mathrm{H}), 5.07-5.06(\mathrm{~d}, J=2.61$ $\mathrm{Hz}, 1 \mathrm{H}), 5.04-5.03(\mathrm{~d}, J=2.79 \mathrm{~Hz}, 1 \mathrm{H}), 4.65-4.54(\mathrm{dd}, J=11.99,41.37 \mathrm{~Hz}, 2 \mathrm{H}), 4.17-4.14(\mathrm{~m}, 3 \mathrm{H}), 3.96-3.95$ (m, 1H), 3.72-3.63 (m, 4H), 3.49-3.47 (dd, $J=2.56,5.14 \mathrm{~Hz}, 1 \mathrm{H}), 1.96-1.90(\mathrm{~m}, 2 \mathrm{H}), 1.73-1.72(\mathrm{~m}, 2 \mathrm{H}), 1.54-$ $1.53(\mathrm{~m}, 1 \mathrm{H}), 1.41-1.16(\mathrm{~m}, 17 \mathrm{H}) ;{ }^{13} \mathrm{C} \mathrm{NMR}\left(125 \mathrm{MHz}, \mathrm{CDCl}_{3}\right): \delta=138.8,131.4,130.2,129.8,129.7,129.0$, $128.5,128.0,127.9,127.8,127.7,96.3,96.3,95.6,95.2,76.2,76.1,72.5,71.0,700.4,70.4,69.6,66.6,66.0,65.9$, 34.0, 32.4, 25.9, 24.5, 24.4, 18.8, 16.6, 16.4, 16.3; IR (NaCl): v 3040, 2981, 2932, 2858, 1451, 1401, 1379, 1300 , 1193, 1156, 1127, $1097 \mathrm{~cm}^{-1}$; HRMS (ESI) m/z: [M+Na] $]^{+}$Calcd for $\mathrm{C}_{37} \mathrm{H}_{50} \mathrm{NaO}_{9}$ 661.3347; Found 661.3345.

\section{Synthesis of 2,3,6-Trideoxy-tetrasaccharide moiety.}

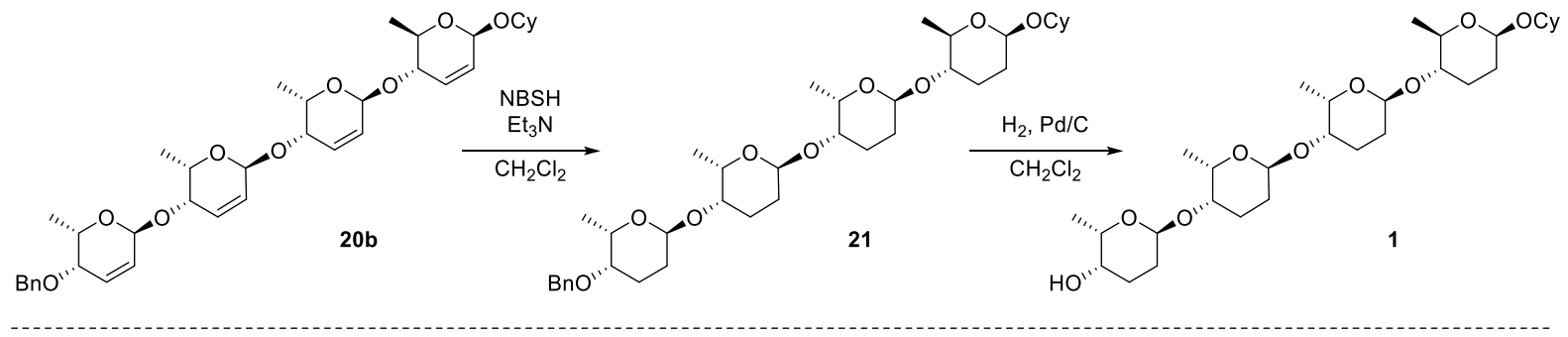




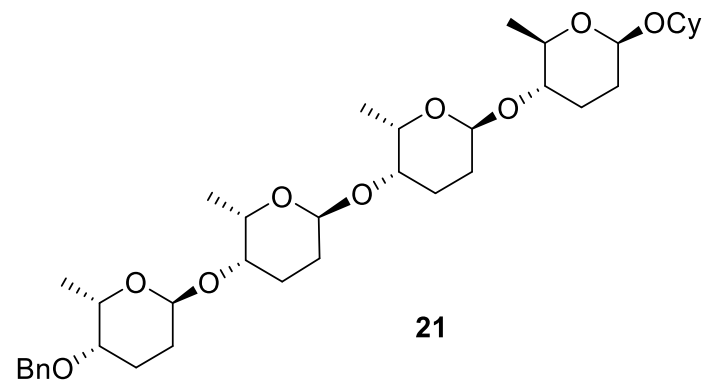

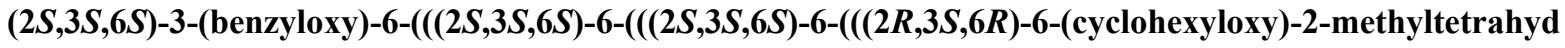
ro-2H-pyran-3-yl)oxy)-2-methyltetrahydro-2H-pyran-3-yl)oxy)-2-methyltetrahydro-2H-pyran-3-yl)oxy)-2 -methyltetrahydro-2H-pyran (21): To a solution of $\mathbf{2 0 b}(29.4 \mathrm{mg}, 0.046 \mathrm{mmol})$ and triethylamine $(0.10 \mathrm{ml}, 0.74$ $\mathrm{mmol})$ in $\mathrm{CH}_{2} \mathrm{Cl}_{2}(0.5 \mathrm{ml})$ was added o-nitrobenzenesulfonylhydrazide ${ }^{2}(159.8 \mathrm{mg}, 0.74 \mathrm{mmol})^{2}$ and the reaction mixture was stirred at room temperature for $24 \mathrm{~h}$. The reaction mixture was diluted with $\mathrm{Et}_{2} \mathrm{O}(20 \mathrm{ml})$ and quenched with saturated $\mathrm{NaHCO}_{3}(20 \mathrm{ml})$, and then the aqueous phase was extracted with $\mathrm{Et}_{2} \mathrm{O}(4 \times 30 \mathrm{ml})$. The organic phase was washed with brine, dried over $\mathrm{Na}_{2} \mathrm{SO}_{4}$ and concentrated under reduced pressure. The crude product was purified by flash column chromatography (Hexane:Ethyl acetate $=80: 20$ ) to afford $\mathbf{2 1}$ as a white solid (27.1 mg, $0.042 \mathrm{mmol}, 91 \%$ yield).

m.p. $187-190{ }^{\circ} \mathrm{C} ; \mathrm{R}_{f} 0.41$ (Hexane:Ethyl acetate $\left.=80: 20\right) ;[\alpha]^{29}{ }_{\mathrm{D}}-59.10\left(c\right.$ 0.925, $\left.\mathrm{CH}_{2} \mathrm{Cl}_{2}\right) ;{ }^{1} \mathrm{H} \mathrm{NMR}(500 \mathrm{MHz}$, $\left.\mathrm{CDCl}_{3}\right): \delta=7.37-7.25(\mathrm{~m}, 5 \mathrm{H}), 4.88-4.83(\mathrm{~m}, 3 \mathrm{H}), 4.69-4.67(\mathrm{~d}, J=12.17 \mathrm{~Hz}, 1 \mathrm{H}), 4.55-4.53(\mathrm{~d}, J=9.03 \mathrm{~Hz}$, $1 \mathrm{H}), 4.45-4.42(\mathrm{~d}, J=12.17 \mathrm{~Hz}, 1 \mathrm{H}), 4.01-3.91(\mathrm{~m}, 3 \mathrm{H}), 3.64-3.60(\mathrm{~m}, 1 \mathrm{H}), 3.50-3.46(\mathrm{~d}, J=20.29 \mathrm{~Hz}, 2 \mathrm{H}), 3.36-$ $3.28(\mathrm{~m}, 2 \mathrm{H}), 3.21-3.17(\mathrm{~m}, 1 \mathrm{H}), 2.11-1.48(\mathrm{~m}, 21 \mathrm{H}), 1.38-1.10(\mathrm{~m}, 17 \mathrm{H}) ;{ }^{13} \mathrm{C} \mathrm{NMR}\left(125 \mathrm{MHz}, \mathrm{CDCl}_{3}\right): \delta=138.9$, $128.4,128.0,127.7,99.7,99.6,99.4,99.2,79.0,76.2,74.9,74.8,74.8,73.8,71.0,67.2,66.9,33.9,32.2,31.5$, $30.0,25.9,25.0,24.9,24.9,24.7,24.6,24.4,24.4,21.7,18.5,17.5,17.4,17.4$; IR (NaCl): v 2978, 2931, 2896, 2896, 2857, 1649, 1588, 1497, 1449, 1399, 1380, $1361 \mathrm{~cm}^{-1}$; HRMS (ESI) m/z: [M+Na] Calcd for $\mathrm{C}_{37} \mathrm{H}_{58} \mathrm{NaO}_{9}$ 669.3973; Found 669.3976.

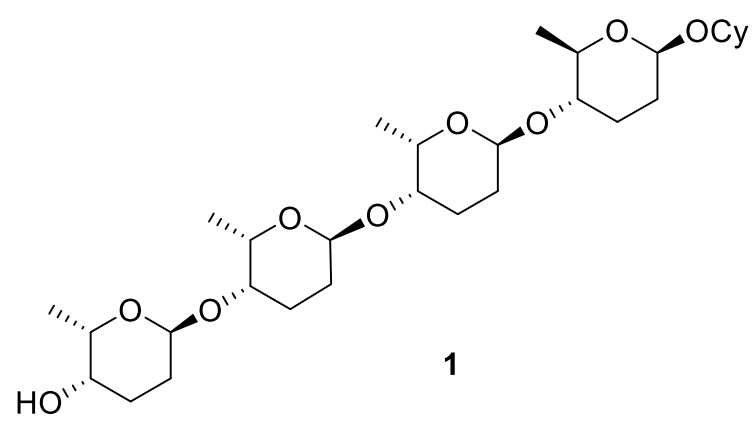

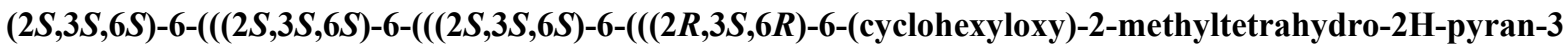
-yl)oxy)-2-methyltetrahydro-2H-pyran-3-yl)oxy)-2-methyltetrahydro-2H-pyran-3-yl)oxy)-2-methyltetrahy dro-2H-pyran-3-ol (1): 21 (23.9 mg, $0.037 \mathrm{mmol})$ was dissolved in THF (0.8 ml), palladium catalyst $(10 \% \mathrm{Pd} / \mathrm{C}$, $7.3 \mathrm{mg}, 30 \mathrm{wt} \%$ ) was added and the reaction mixture was stirred under $\mathrm{H}_{2}(1 \mathrm{~atm})$ for $30 \mathrm{~min}$ at room temperature. The crude mixture was filtered with a pad of celite (rinse; $\mathrm{CH}_{2} \mathrm{Cl}_{2} 50 \mathrm{ml}$ ), and then concentrated under reduced pressure. the crude product was purified by flash column chromatography (Pentane:Ethyl acetate $=70: 30$ ) to afford 1 as a white solid $(20.1 \mathrm{mg}, 0.036 \mathrm{mmol}, 98 \%)$.

m.p. 192-195 ${ }^{\circ} \mathrm{C} ; \mathrm{R}_{f} 0.28$ (Hexane:Ethyl acetate = 50:50); $[\alpha]^{29} \mathrm{D}-111.62\left(c 0.95, \mathrm{CH}_{2} \mathrm{Cl}_{2}\right) ;{ }^{1} \mathrm{H} \mathrm{NMR}(600 \mathrm{MHz}$, $\left.\mathrm{CDCl}_{3}\right): \delta=4.89-4.88$ (brs, $\left.1 \mathrm{H}\right), 4.85-4.84(\mathrm{~d}, J=2.99 \mathrm{~Hz}, 1 \mathrm{H}), 4.83-4.82(\mathrm{~d}, J=3.56 \mathrm{~Hz}, 1 \mathrm{H}), 4.55-4.53(\mathrm{dd}, J$ $=1.92,9.25 \mathrm{~Hz}, 1 \mathrm{H}), 4.07-4.03(\mathrm{q}, J=6.62 \mathrm{~Hz}, 1 \mathrm{H}), 3.98-3.92(\mathrm{~m}, 2 \mathrm{H}), 3.65-3.60(\mathrm{qd}, J=3.92,9.39 \mathrm{~Hz}, 1 \mathrm{H})$, 3.59-3.57 (m, 1H), 3.49 (brs, $1 \mathrm{H}), 3.47$ (brs, $1 \mathrm{H}), 3.34-3.32$ (qd, $J=6.19,8.87 \mathrm{~Hz}, 1 \mathrm{H}), 3.22-3.17$ (ddd, $J=4.17$, 9.29, $10.51 \mathrm{~Hz} 1 \mathrm{H}), 2.11-2.05(\mathrm{~m}, 3 \mathrm{H}), 2.02-1.87(\mathrm{~m}, 6 \mathrm{H}), 1.81-1.66(\mathrm{~m}, 9 \mathrm{H}), 1.61-1.50(\mathrm{~m}, 4 \mathrm{H}), 1.37-1.32(\mathrm{~m}$, 
$1 \mathrm{H}), 1.30-1.22(\mathrm{~m}, 7 \mathrm{H}), 1.20-1.15(\mathrm{~m}, 4 \mathrm{H}), 1.12-1.10(\mathrm{~d}, J=6.60 \mathrm{~Hz} 3 \mathrm{H}), 1.12-1.10(\mathrm{~d}, J=6.60 \mathrm{~Hz} 3 \mathrm{H}) ;{ }^{13} \mathrm{C}$ NMR (150 MHz, $\left.\mathrm{CDCl}_{3}\right): \delta=99.8,99.6,99.4,99.2,79.1,76.2,75.1,74.9,74.8,67.7,67.2,67.0,66.9,33.9,32.2$ $31.5,30.0,26.2,25.9,25.1,24.9,24.7,24.6,24.4,23.8,18.5,17.4,17.4,17.3$; IR (NaCl): v 3475, 2933, 2858, 1648, 1448, 1380, 1362, 1322, 1286, 1213, 1200, $1166 \mathrm{~cm}^{-1}$; HRMS (ESI) m/z: $[\mathrm{M}+\mathrm{Na}]^{+}$Calcd for $\mathrm{C}_{30} \mathrm{H}_{52} \mathrm{NaO}_{9}$ 579.3504; Found 579.3505.

\section{Reference}

1. Lee, J.; Kang, S.; Kim, J.; Moon, D.; Rhee, Y. H., Angew. Chem. Int. Ed. 2019, 58, 628631.

2. Babu, R. S.; Guppi, S. R.; O'Doherty, G. A., Org. Lett. 2006, 8, 1605-1608 


\section{9. ${ }^{1} \mathrm{H},{ }^{13} \mathrm{C}$ NMR and $2 \mathrm{D}$ NMR of new compounds}

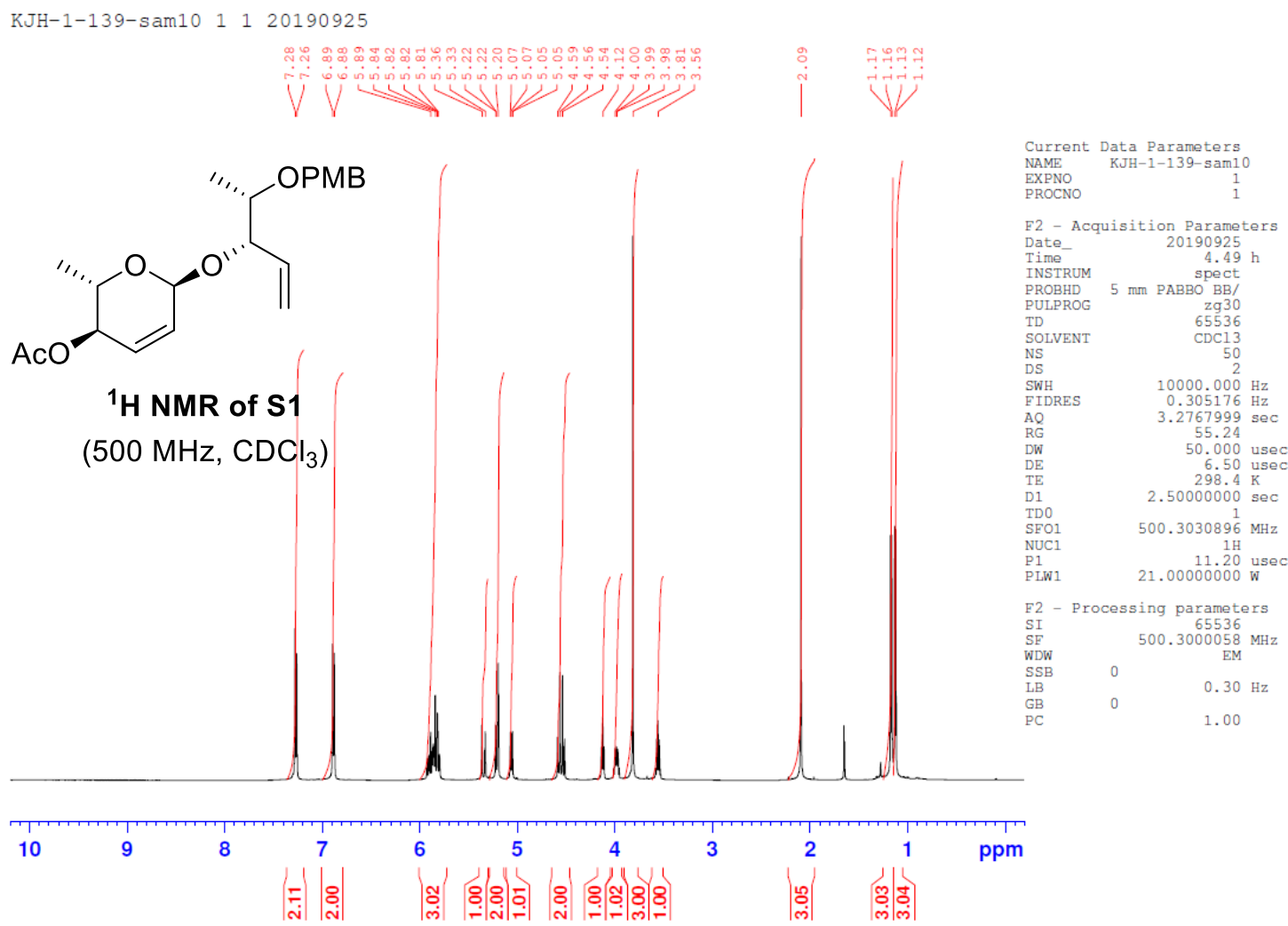

KJH-1-139-sam10 21320190925

${ }^{13} \mathrm{C}$ NMR of $\mathrm{S1}$ $\left(125 \mathrm{MHz}, \mathrm{CDCl}_{3}\right)$<smiles>C=C[C@@H](O[C@H]1C=C[C@@H](OC(C)=O)[C@@H](C)O1)[C@@H](C)OCC</smiles>

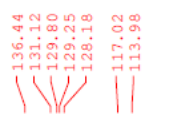

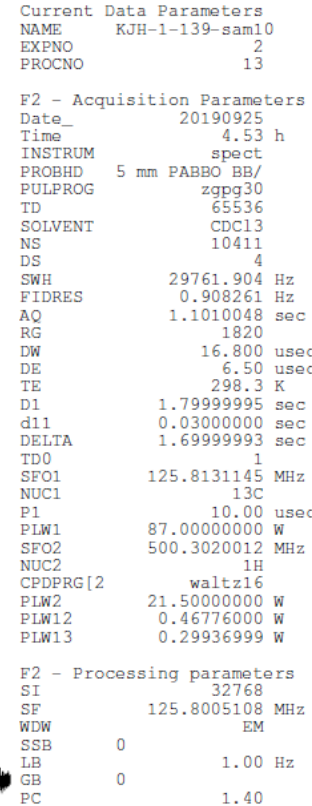



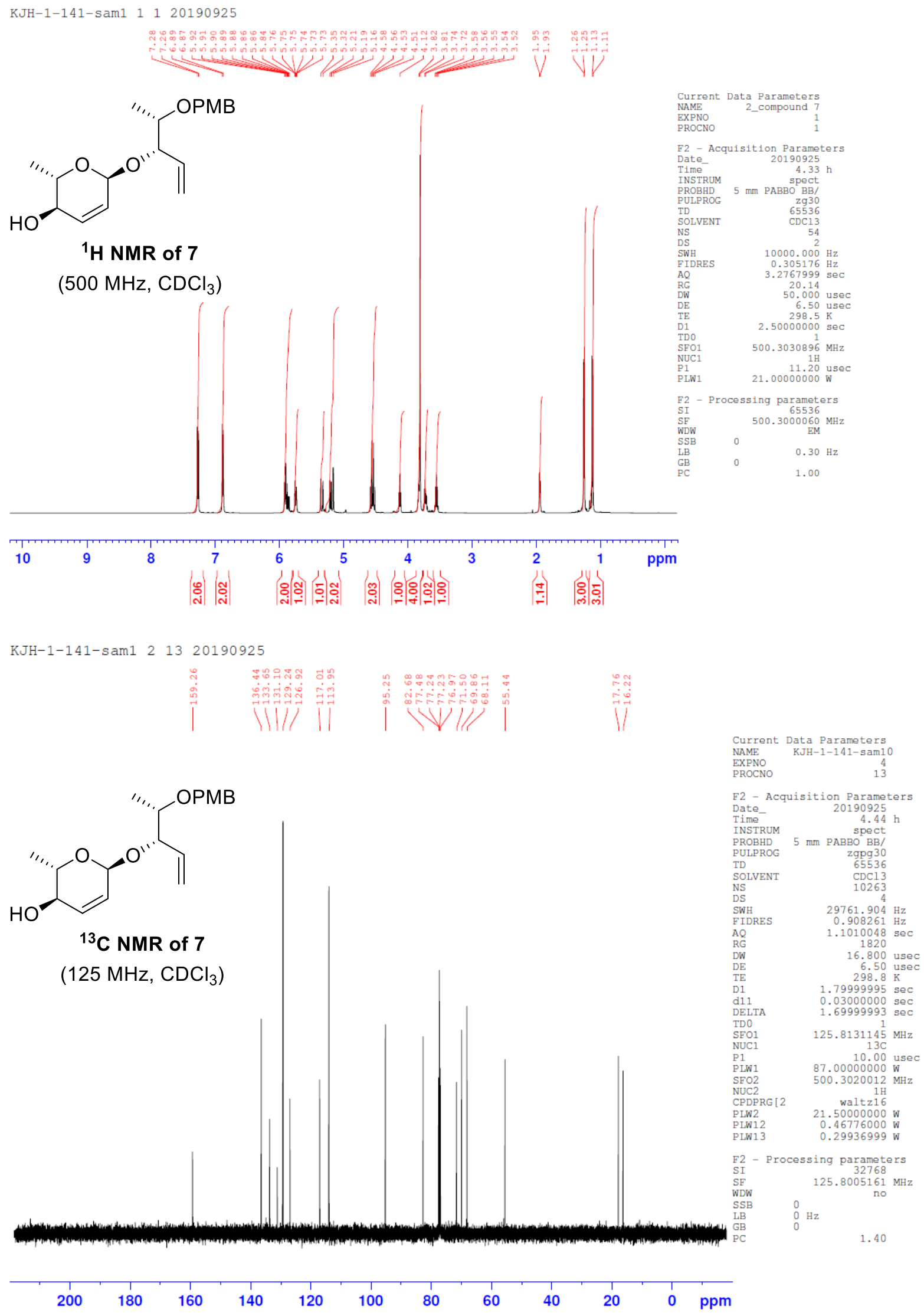


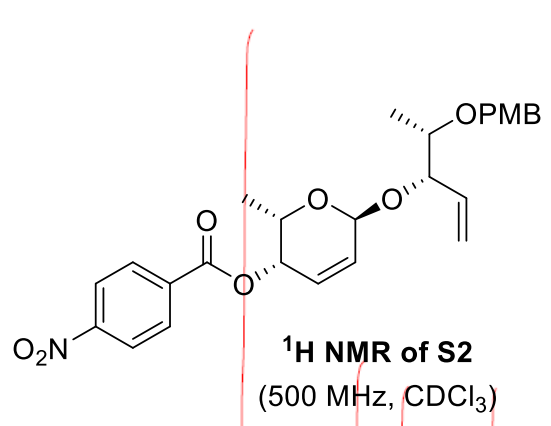
$\left(500 \mathrm{MHz}, \mathrm{CDCl}_{3}\right)$

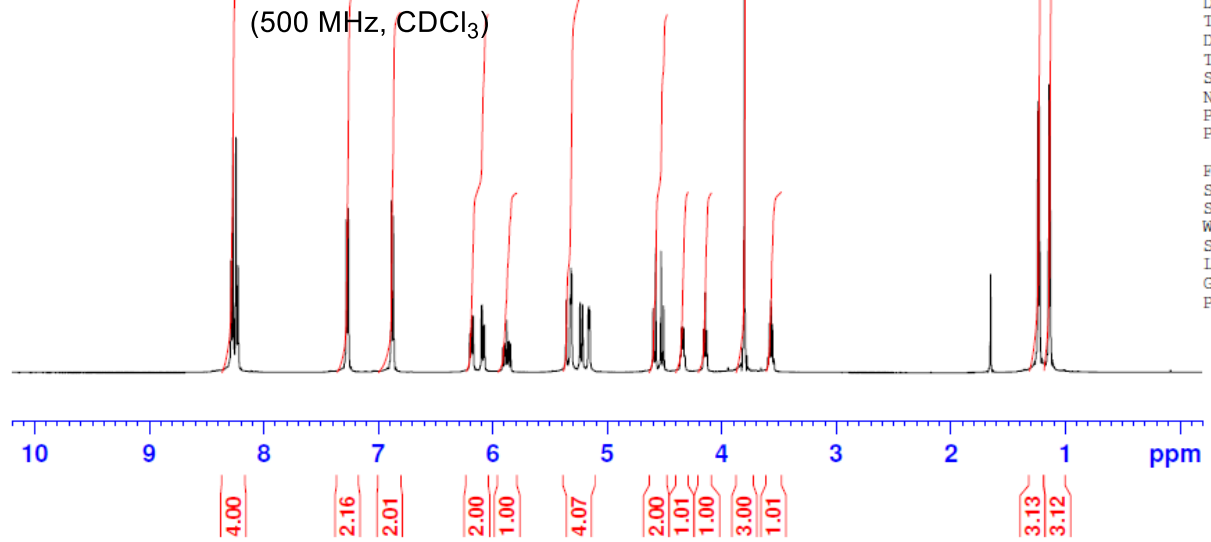

KJH-1-142-sam2 21320190627

सि.

|ं।<smiles>C=C[C@H](O[C@H]1C=C[C@@H](OC(=O)c2ccc([N+](=O)[O-])cc2)[C@@H](C)O1)[C@@H](C)O[Mg]</smiles>

$\left(125 \mathrm{MHz}, \mathrm{CDCl}_{3}\right)$

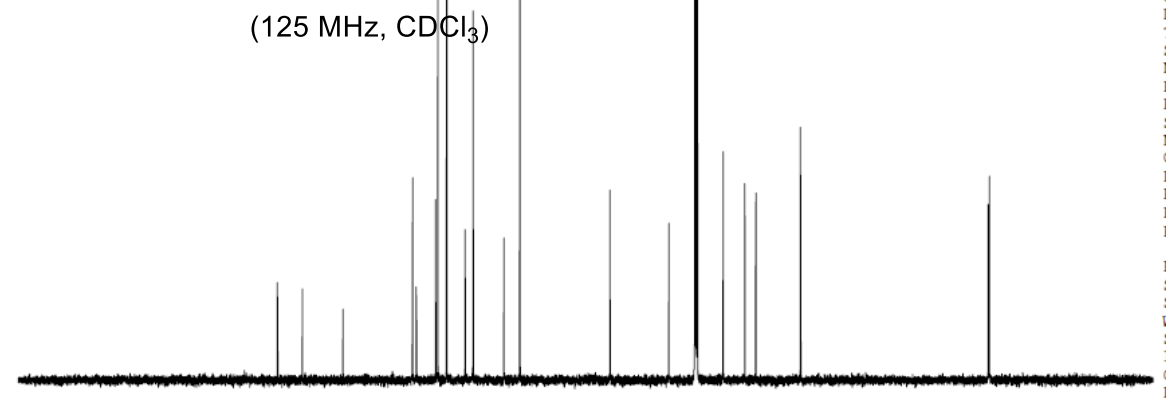

200

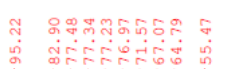

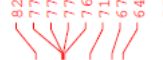

Current. Data Parameters
NAME
KJH-1-142-sam2 EXPNO
PROCNO

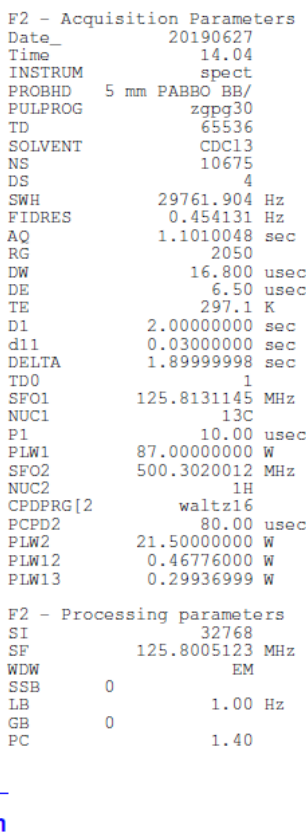

Current Data Parameters EXPNO F2 - Acquisition Parameters $\begin{array}{lr} & 20190627 \\ \text { Date_- } & 14.02 \mathrm{~h}\end{array}$ spect
PROBHD $5 \mathrm{~mm}$ PABBO PULPROG
zg30

SOLVENT

$\mathrm{CDC} 13$
35

$\begin{array}{lr}\text { SWH } & 10000.000 \mathrm{~Hz} \\ \text { FIDRES } & 0.305176 \mathrm{~Hz}\end{array}$

$\begin{array}{lr}\text { AQ } & 3.2767999 \mathrm{sec} \\ \text { RG } & 55.24\end{array}$

$\begin{array}{lr}\text { DW } & 50.000 \text { usec } \\ D E & 6.50 \text { usec }\end{array}$

$\begin{array}{ll}297.1 \mathrm{~K} \\ \mathrm{D} 1 & 2.00000000 \mathrm{sec}\end{array}$

$\quad 500.3030896 \mathrm{MHz}$

$\begin{array}{ll}\text { NUC1 } & 1 \mathrm{H} \\ \text { P1 } & 11.20 \text { usec } \\ \text { PLW1 } & 21.00000000 \mathrm{~W}\end{array}$

F2 - Processing parameters

SI $\quad 500.3000120 \mathrm{MHz}$

$\underset{\mathrm{SSB}}{\mathrm{SSW}} 0.30 \mathrm{~Hz}$

$\begin{array}{lll}\mathrm{CB} & 0.30 \\ \mathrm{~GB} & 0 & 1.00\end{array}$ 
<smiles>C=C[C@@H](O[C@H]1C=C[C@@H](O)[C@@H](C)O1)[C@@H](C)OCC</smiles>

${ }^{1} \mathrm{H}$ NMR of 8 (500 MHz, $\mathrm{CDCl}_{3}$ )
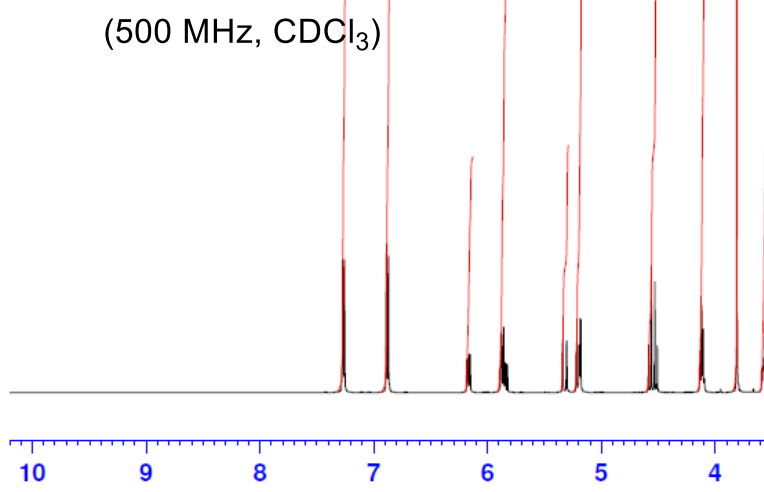

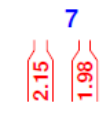

|ำ
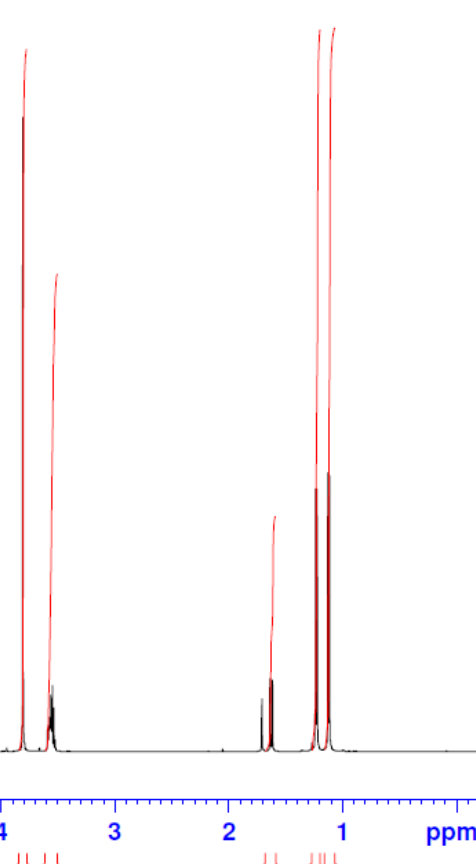

Current Data Parameters

EXPNO

F2 - Acquisition Parameters
Date_ 20190712
Time-

Time-

spect
PRSTRUM
PUOBHD $5 \mathrm{~mm}$ PABBO BB/

TD

NS

$\begin{array}{lr}\text { DS } & 2 \\ \text { SWH } & 10000.000 \mathrm{~Hz}\end{array}$

AQ $\quad 3.2767999 \mathrm{sec}$

55.24
$\mathrm{DW}$$\quad 50.000$ use

$\begin{array}{lr}6.50 \mathrm{u} \\ \mathrm{TE} & 2.00000000 \mathrm{~K}\end{array}$

SFO1 $\quad 500.3030896 \mathrm{MHz}$

$\begin{array}{ll}\text { NUC1 } & 1 \mathrm{H} \\ \text { P1 } & 11.20 \text { usec } \\ \text { PLW1 } & 21.00000000 \text { W }\end{array}$

F2 - Processing parameters

$\begin{array}{lr}\text { SI } & 65536 \\ \text { SF } & 500.3000077 \mathrm{MHz}\end{array}$

SSB $\quad 0 \quad$ EM

$\begin{array}{lll}\mathrm{SSB} & 0 & 0.30 \\ \mathrm{CB} & 0 & \end{array}$

KJH-1-144-samo 21320190712

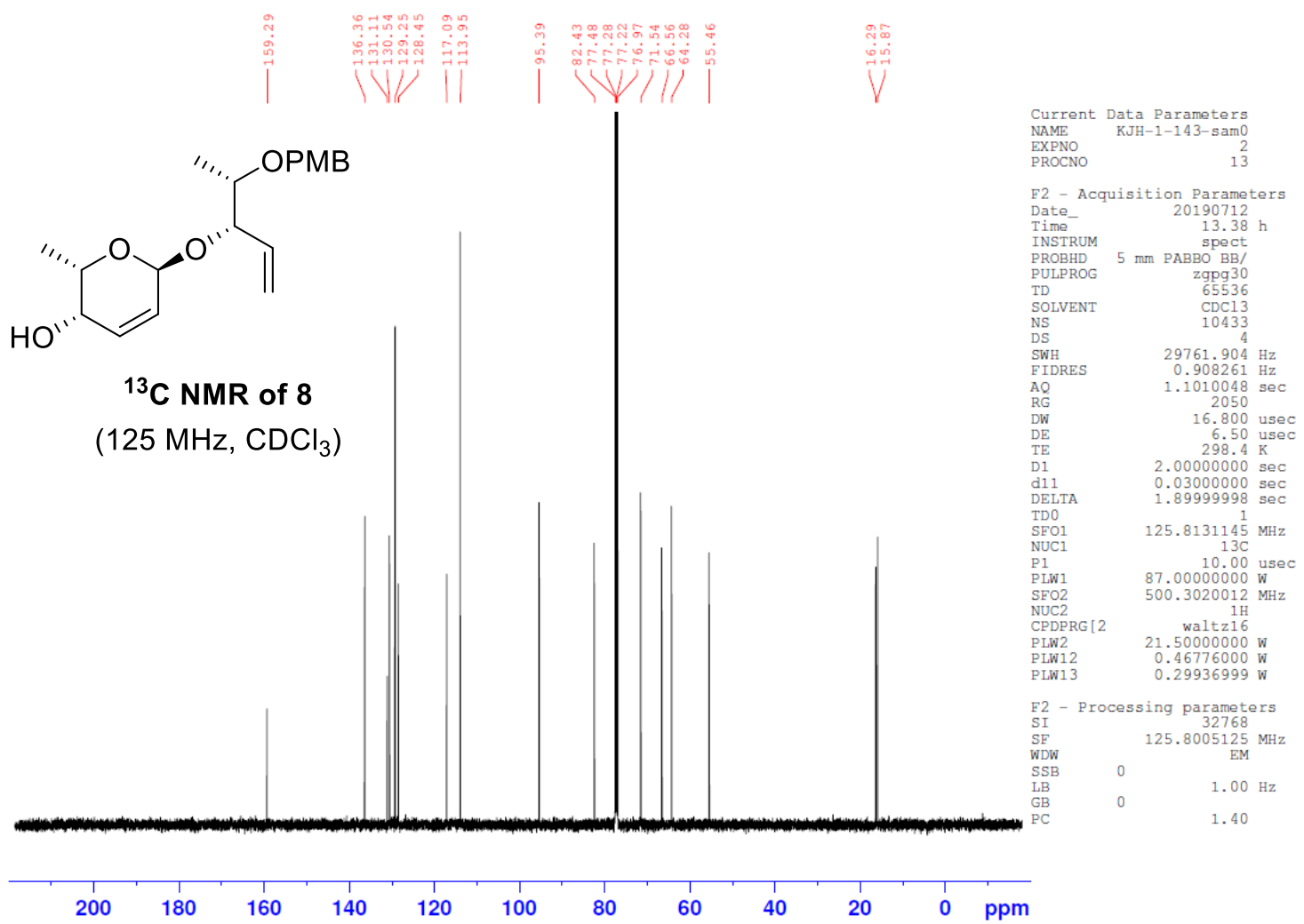




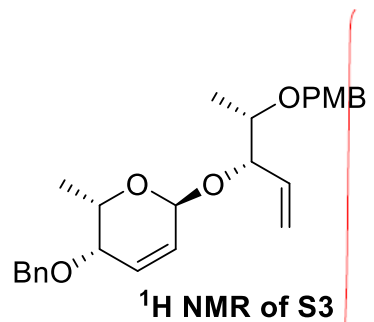

${ }^{1} \mathrm{H}$ NMR of S3

$\left(500 \mathrm{MHz}, \mathrm{CDCl}_{\beta}\right)$

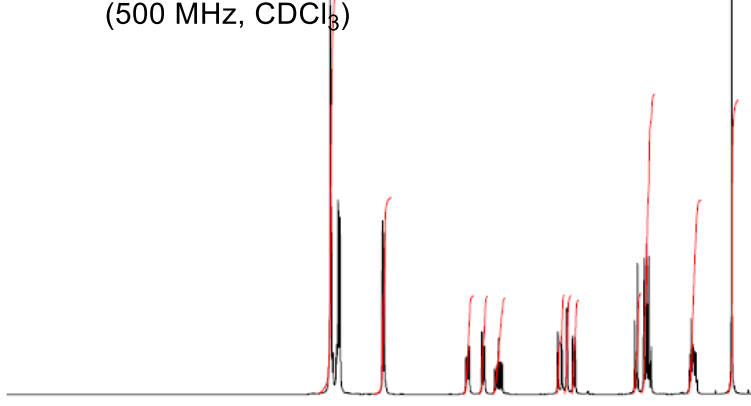

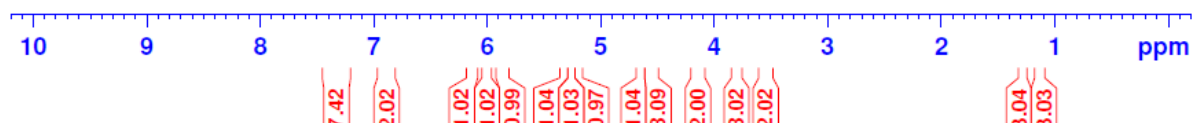

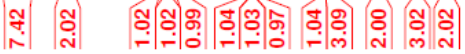

KJH-1-144-sam0 21320190712

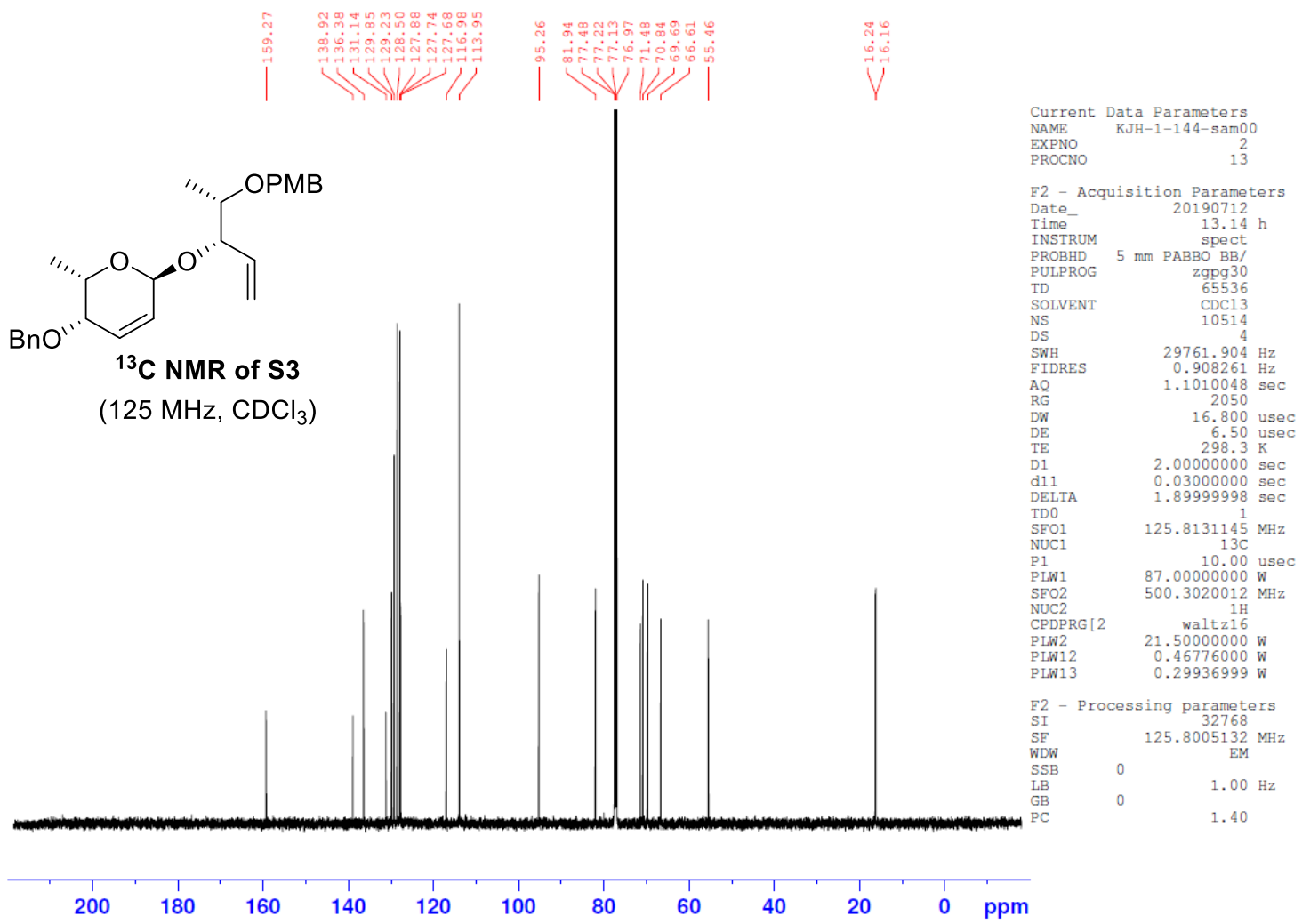

Current Data Parameters
NAME EXPNO F2 - Acquisition Parameters
Date_ 20190712
Time
TNSTh

Time PROBHD $5 \mathrm{~mm}$ PABBO BB/

SOLVENT

\begin{tabular}{ll} 
AQ & $3.305176 \mathrm{~Hz}$ \\
\hline & $52767999 \mathrm{sec}$
\end{tabular}

50.000 usec
6.50 usec

$\begin{array}{rl}297.7 & \mathrm{~K} \\ 2.00000000 \mathrm{sec}\end{array}$

$500.3030896 \mathrm{MH}$ $1 \mathrm{H}$
1.20 use

2 - Processing parameters

SI $\quad 500.3000114 \mathrm{MH}$
SWH $\quad 10000.000 \mathrm{~Hz}$ $21.00000000 \mathrm{~W}$ 


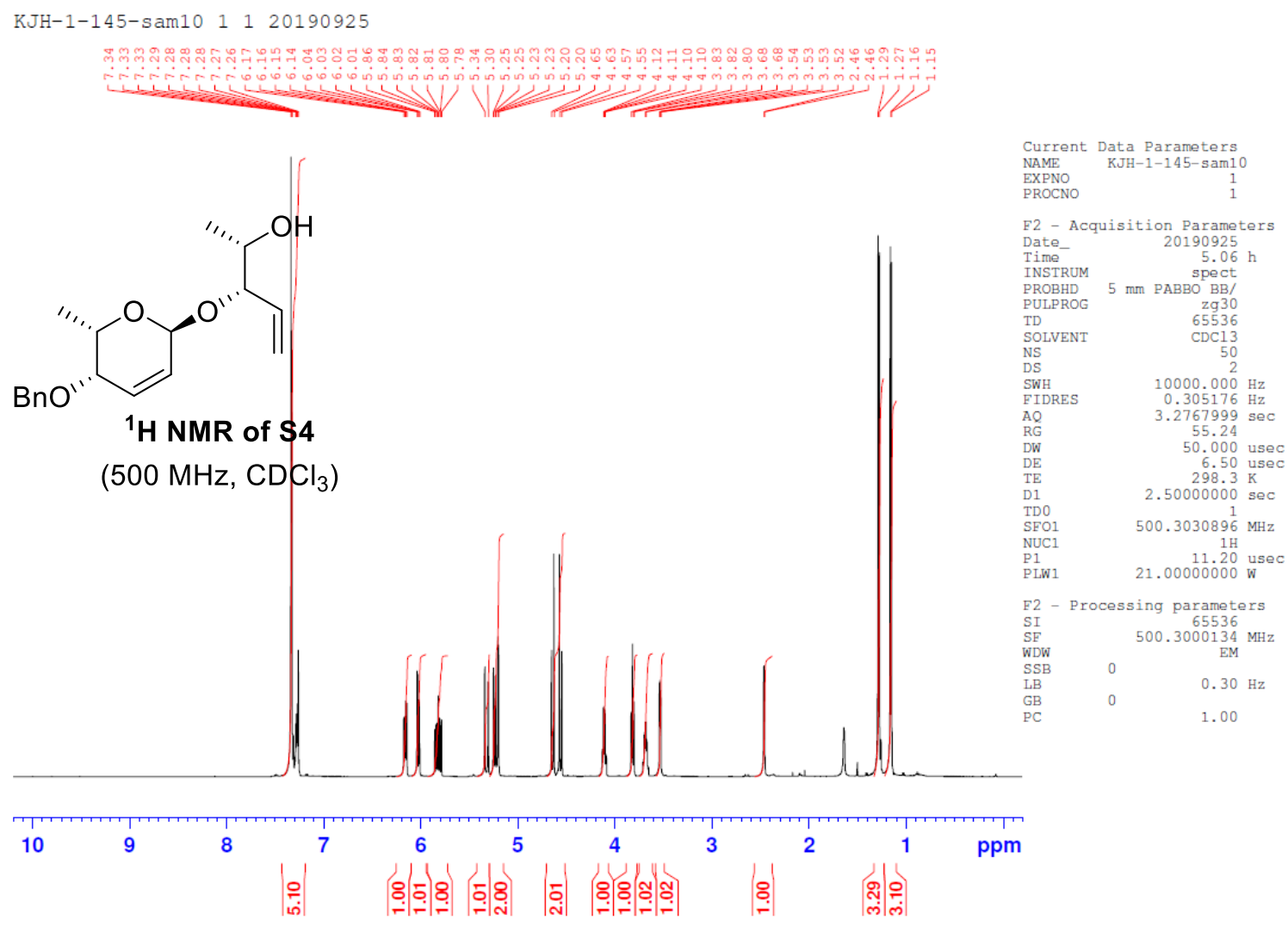

KJH-1-145-sam10 21320190925

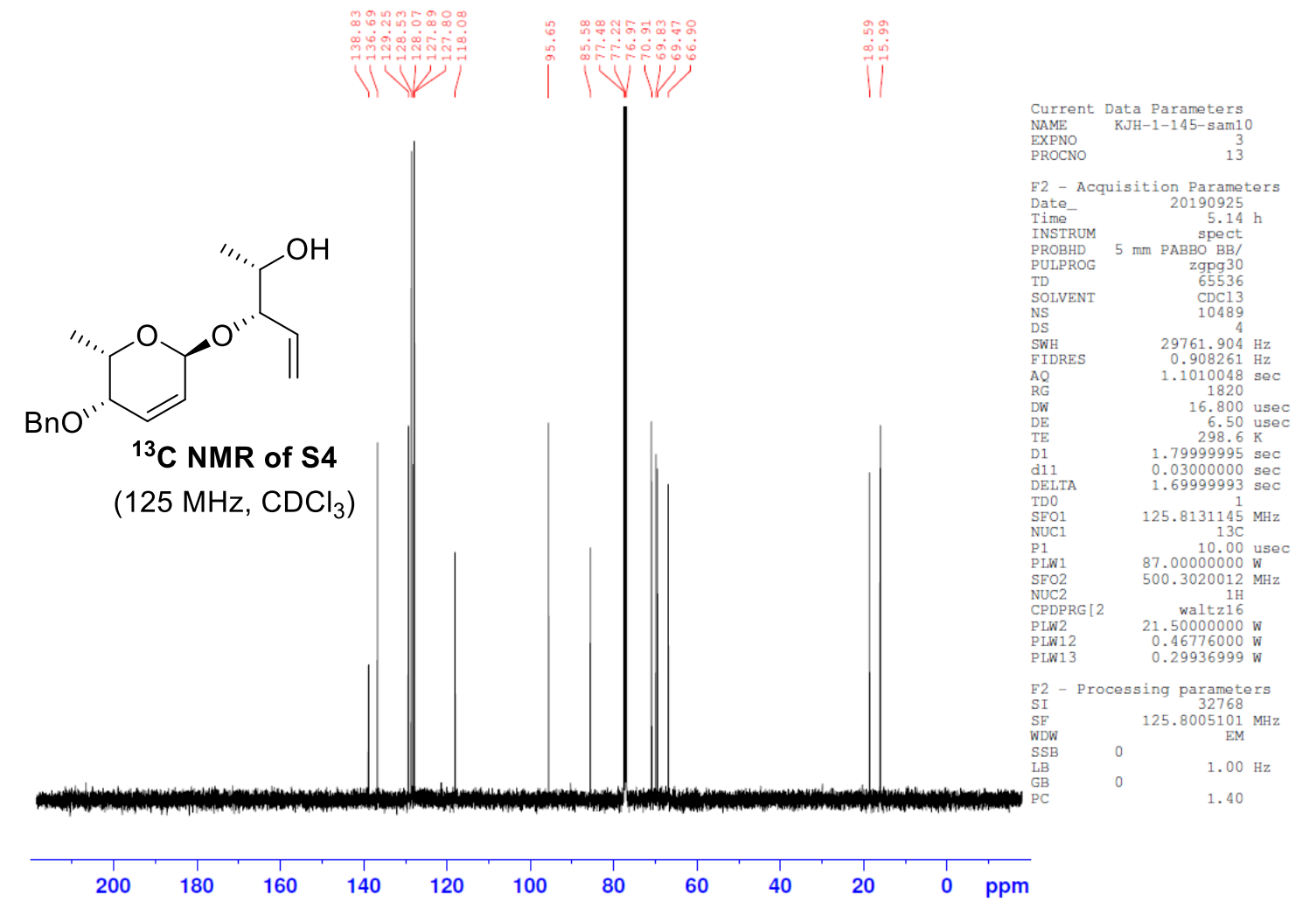




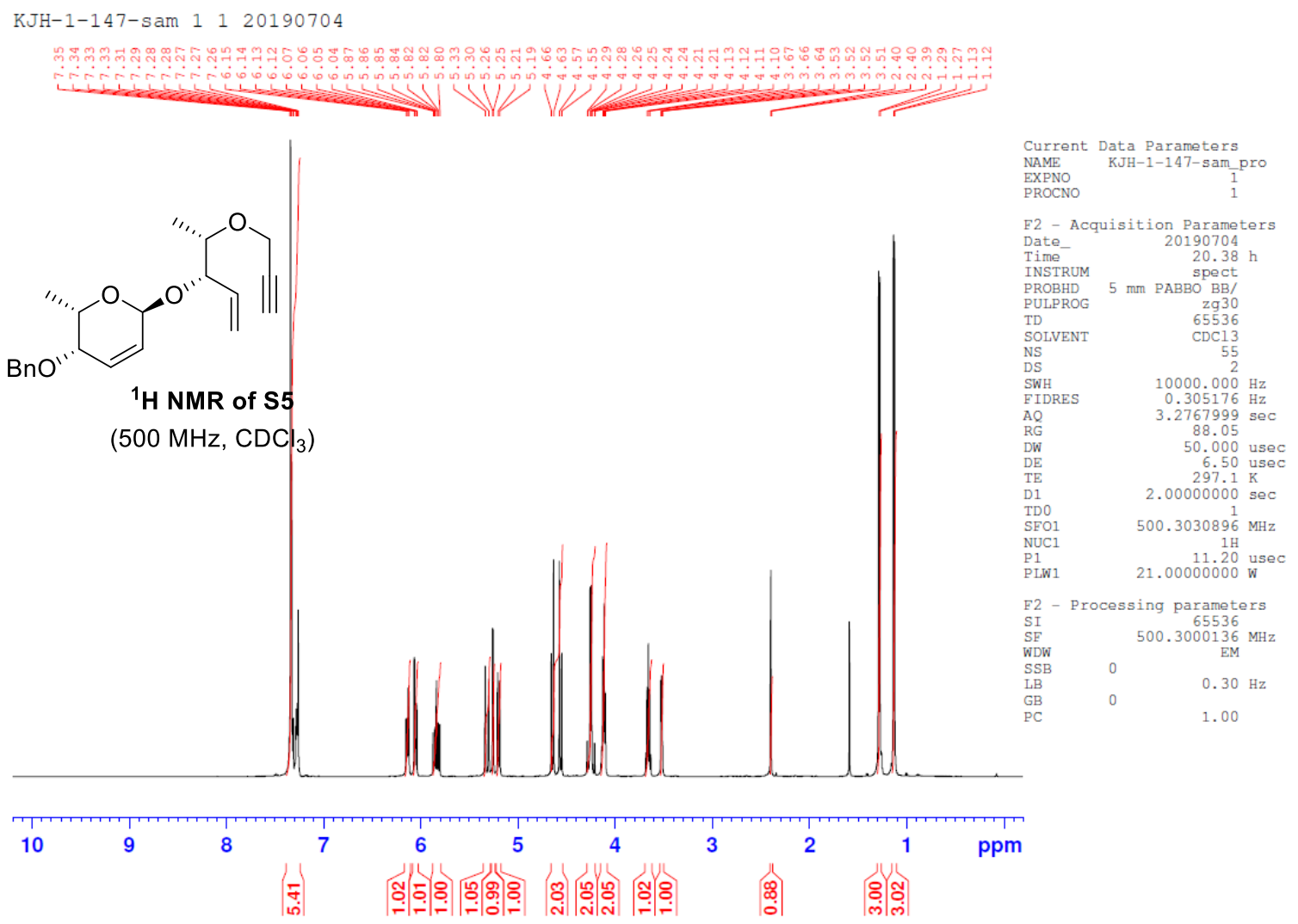

KJH-1-147-samo 31320190712

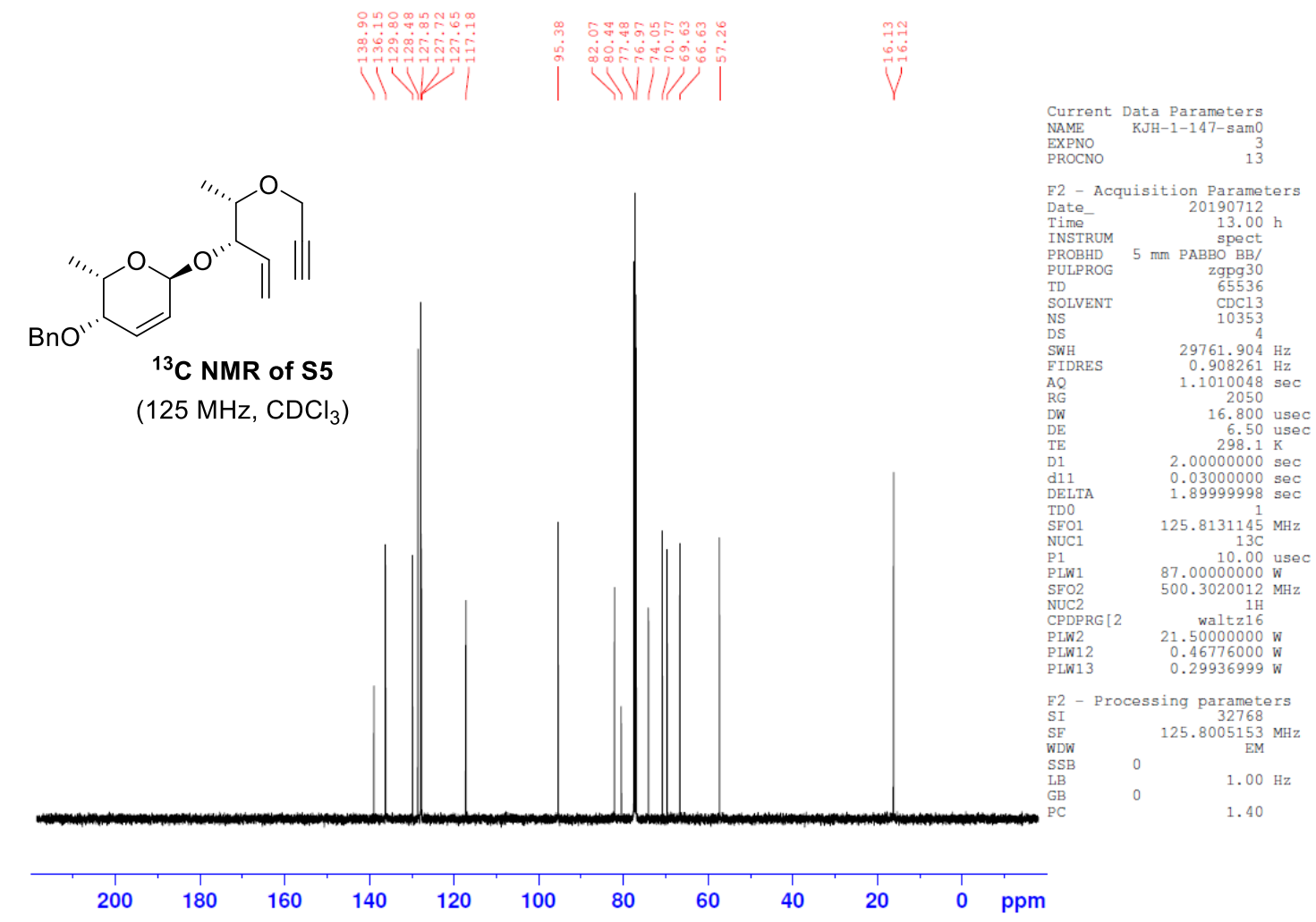




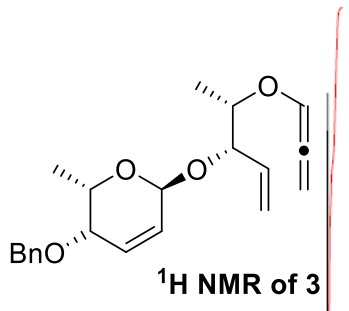

Current Data Parameters
NAME KJH-1-153-sam EXPNO

F2 - Acquisition Parameter
Date_ 20190715

Time

INSTRUM
PROBHD
PULPROG

SOLVENT

$\begin{array}{lr} & 10000.000 \mathrm{~Hz} \\ \text { FIDRES } & 0.305176 \mathrm{~Hz}\end{array}$

(500 $\mathrm{MHz}, \mathrm{CDCl}_{3}$ )

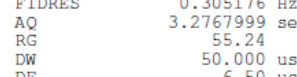

$\begin{array}{lr}\text { DW } & 50.000 \text { usec } \\ \text { DE } & 6.50 \text { usee }\end{array}$

$298.1 \mathrm{~K}$
$\mathrm{TE}$$\quad 2.00000000 \mathrm{sec}$

$\begin{array}{ll}\text { TDO } & 500.3030896 \mathrm{MHz}\end{array}$

NUC1 11.20 use

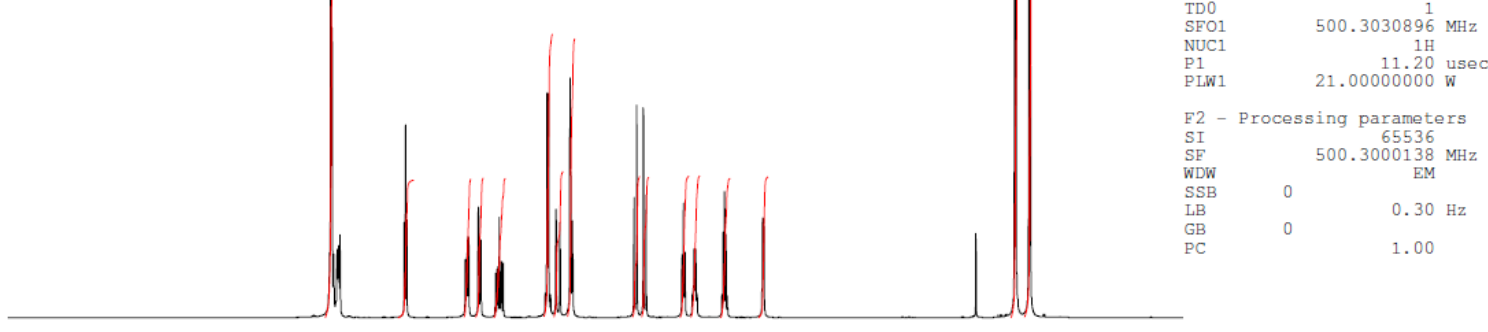

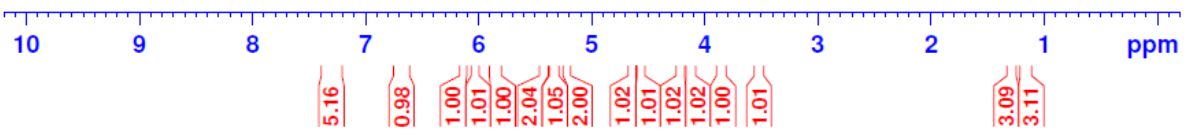

KJH-1-153-sam 21320190715

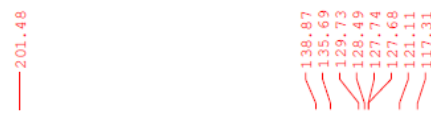

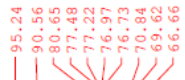

Current Data Parameters
NAME KJH-1-153-sam

$\begin{array}{lr}\text { NAME } & \text { KJH-1-153-sam } \\ \text { EXPNO } & 2 \\ \text { PROCNO } & 13\end{array}$

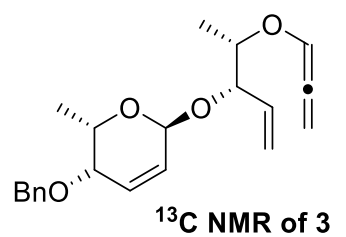

F2 - Acquisition Parameters
Date 20190715

Time-

INSTRUM spect

$\begin{array}{rr}\text { PROBHD } & 5 \mathrm{~mm} \text { PABBO BB/ } \\ \text { PULPROG } & \text { zgpg30 } \\ \text { G } 5536\end{array}$

SOLVENT

10333

$\begin{array}{lr}\text { SWH } & 29761.904 \mathrm{~Hz} \\ \text { FIDRES } & 0.908261 \mathrm{~Hz}\end{array}$

AQ $1.1010048 \mathrm{sec}$

${ }^{13} \mathrm{C}$ NMR of 3

(125 $\mathrm{MHz} \mathrm{CDCl}_{3}$ )

\begin{tabular}{l} 
RG \\
DW \\
DE \\
TE \\
D \\
d \\
D \\
D \\
S \\
N \\
D \\
P \\
P \\
S \\
N \\
C \\
P \\
P \\
P \\
P \\
E \\
S \\
S \\
W \\
S \\
I \\
C \\
\hline
\end{tabular}

16.800 usec

$298.2 \mathrm{~K}$

$2.00000000 \mathrm{sec}$
$0.03000000 \mathrm{sec}$

$1.89999998 \mathrm{sec}$

125.8131145 $\mathrm{MHz}$

10.00 usec

$500.3020012 \mathrm{MH}$

Waltz16
CPDPRG [2
$21.50000000 \mathrm{~W}$

$\begin{array}{lr}\text { PLW2 } & 21.50000000 \mathrm{~W} \\ \text { PLW12 } & 0.46776000 \mathrm{~W} \\ \text { PLW13 } & 0.29936999 \mathrm{~W}\end{array}$

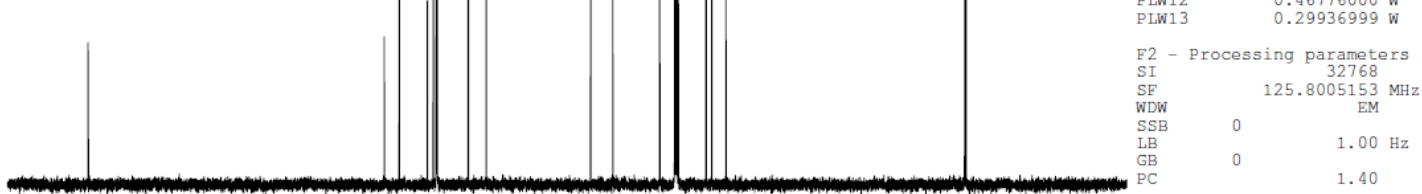

200

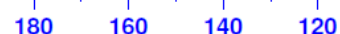

100

60

20

ppm 
$\underbrace{\infty}$

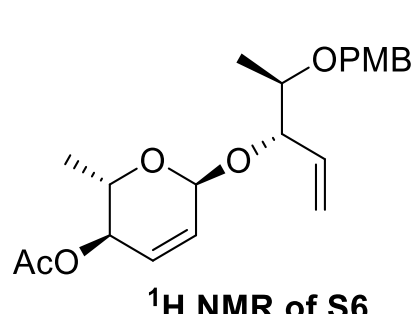

NMR of $\mathbf{S 6}$

$\left(500 \mathrm{MHz}, \mathrm{CDCl}_{3}\right)$

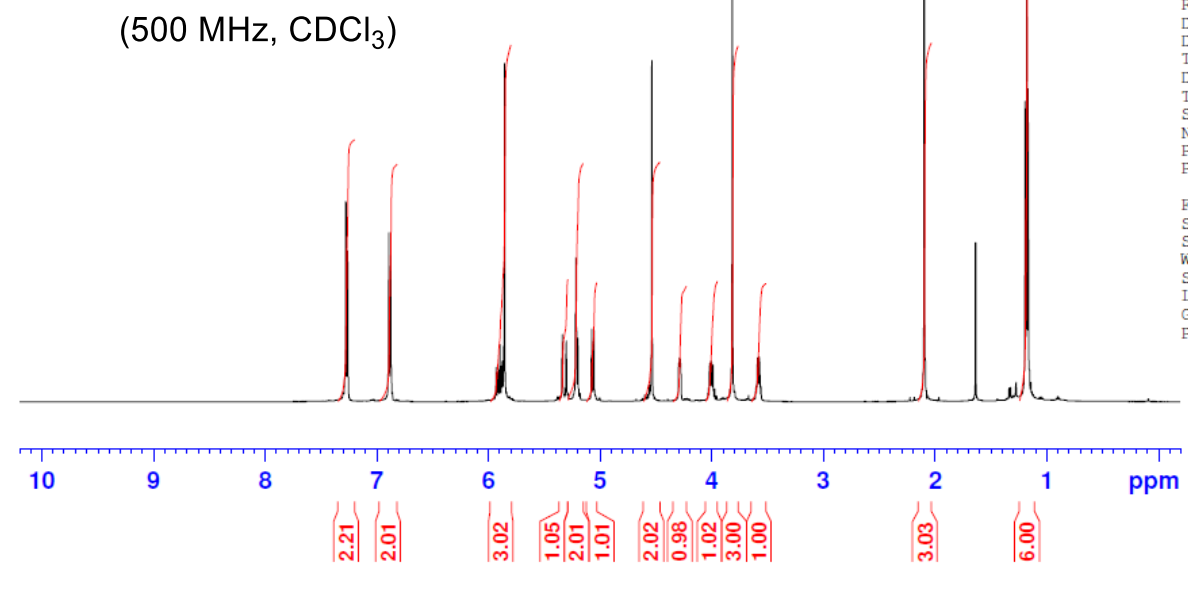

KJH-1-135-sam 21320190923

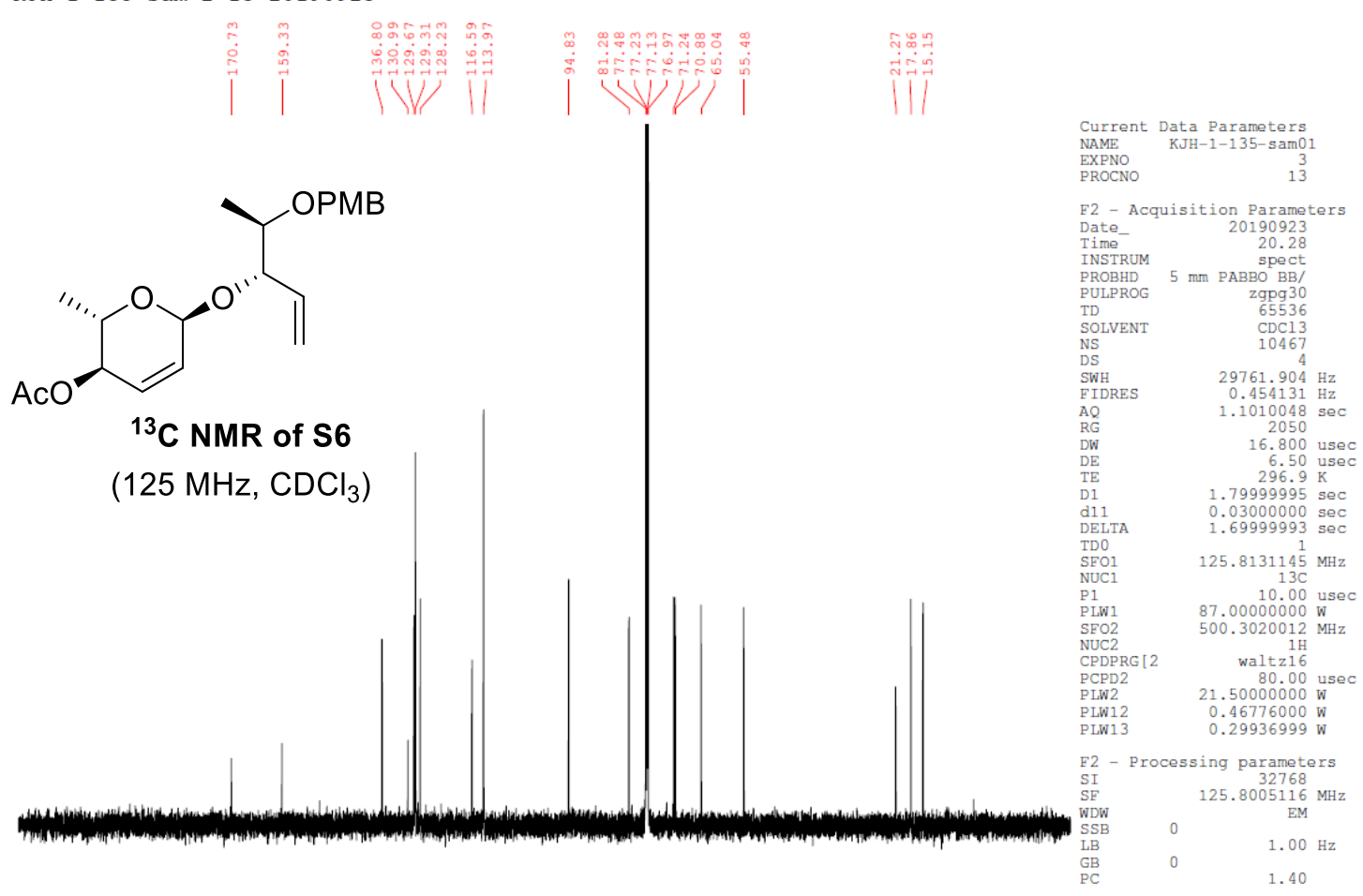

(125 MHz, $\mathrm{CDCl}_{3}$ )

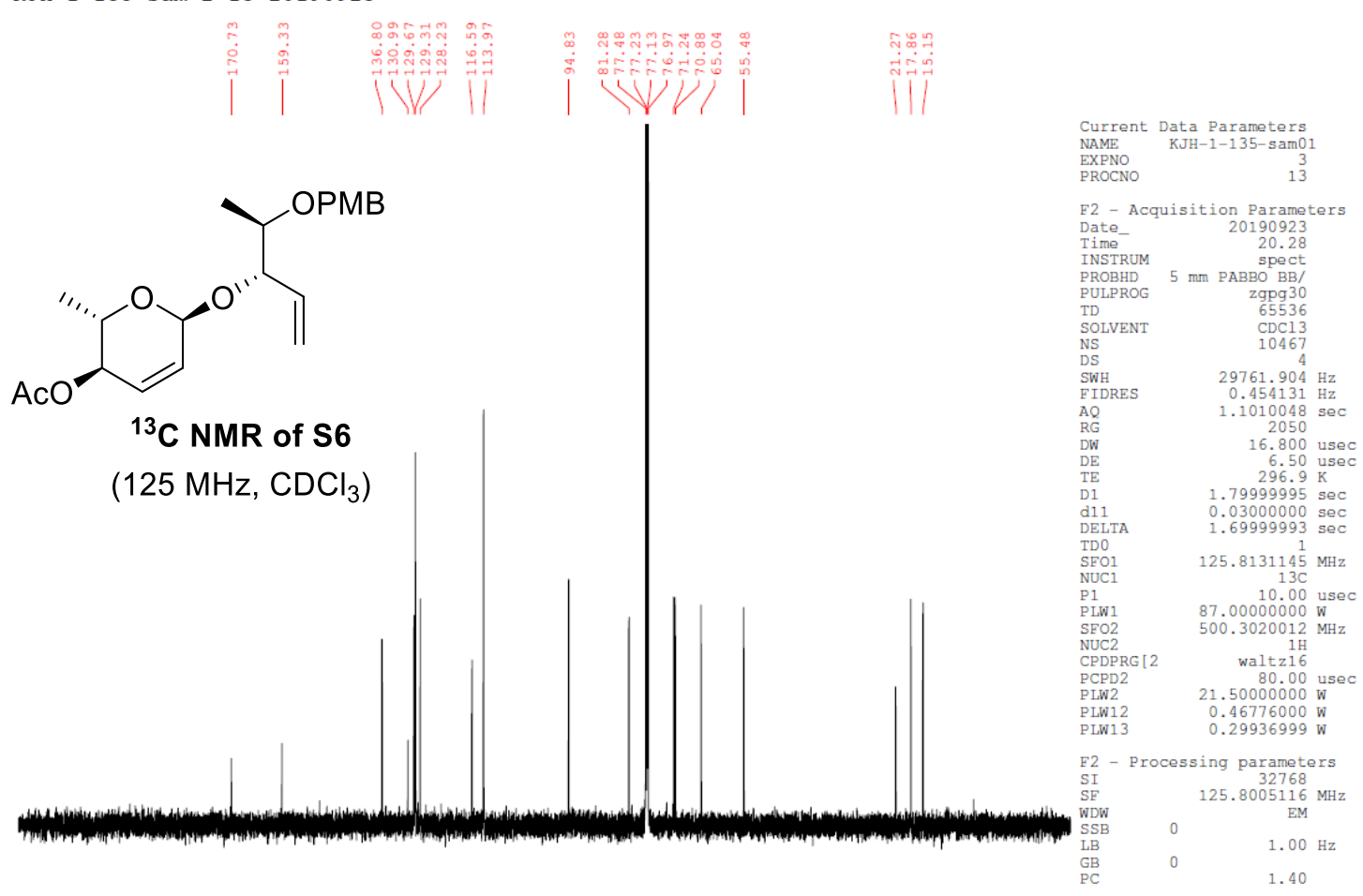

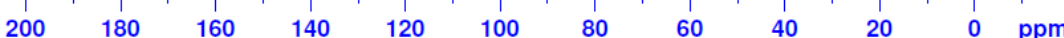

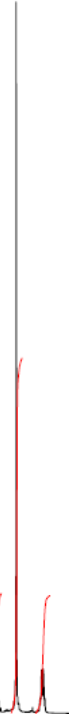

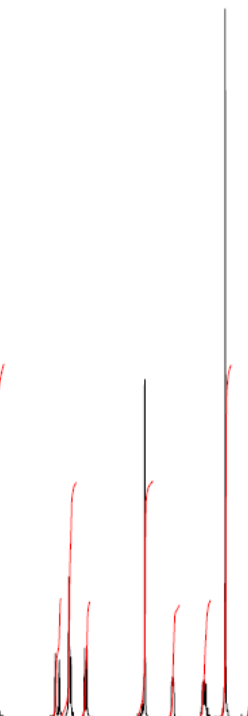

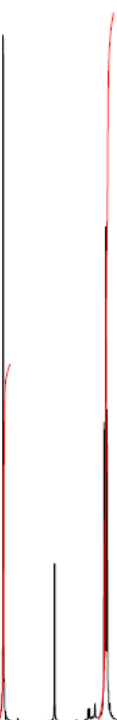

Current Data Parameters

NAME

F2 - Acquisition Parameter

$\begin{array}{lr}\text { F2 - Acquisition Parameters } \\ \text { Date_- } & 20190923 \\ \text { Time } & 20.44 \mathrm{~h}\end{array}$

$\begin{array}{r}\text { spect } \\ \text { INSTRUM } \\ \text { PROBHD }\end{array}$
PUIP $\mathrm{mm}$ PABBO BB/

PULPROG
TD

NS

$\begin{array}{lr}\text { DS } & 10000.000 \mathrm{~Hz} \\ \text { SWH } & 0.305176 \mathrm{~Hz} \\ \text { FIDRES } & 3.2767999 \mathrm{~Hz}\end{array}$

$\mathrm{AQ} \quad 3.2767999 \mathrm{se}$

DW

6.50 use
$298.0 \mathrm{~K}$

$\quad 2.50000000 \mathrm{sec}$

UC1

PLW1

$21.00000000 \mathrm{~W}$

F2 - Processing parameters

$\begin{array}{ll}\text { SE } & 500.3000039 \\ \text { WDW } & \end{array}$

$\mathrm{SS}$
$\mathrm{LB}$
$\mathrm{GB}$

$0.30 \mathrm{~Hz}$ 
<smiles>[R16]OC(C)[C@@H](C=C)O[C@@H]1C=C[C@@H](O)[C@@H](C)O1</smiles>

${ }^{1} \mathrm{H}$ NMR of $\mathrm{S} 7$ $\left(500 \mathrm{MHz}, \mathrm{CDCl}_{3}\right)$
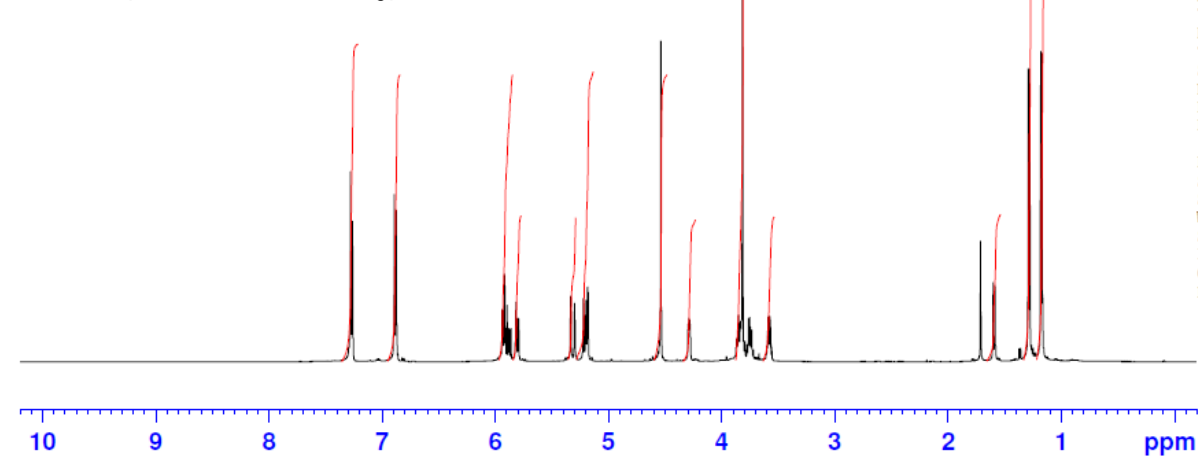

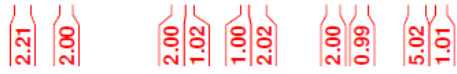

KJH-1-136-sam01 21320190923

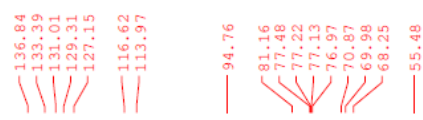<smiles>C=C[C@@H](O[C@@H]1C=C[C@@H](O)[C@H](C)O1)C(C)O[Na]</smiles>

${ }^{13} \mathrm{C}$ NMR of $\mathrm{S} 7$ $\left(125 \mathrm{MHz}, \mathrm{CDCl}_{3}\right)$

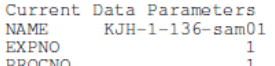
EXPNO F2 - Acquisition Parameters $\begin{array}{lr}\text { Date_- } & 20190923 \\ \text { Time- } & 20.48\end{array}$ INSTRUM spect PULPROG TD SOLVEN

NS
DS
SWH SWH $\quad 10000.000 \mathrm{~Hz}$ $\begin{array}{ll}\text { AQ } & 0.305176 \mathrm{~Hz} \\ \text { AQ } & 3.2767999 \mathrm{sec}\end{array}$ RW $\quad 50.000$ use $\begin{array}{lr}\text { TE } & 297.2 \mathrm{~K} \\ \mathrm{D} 1 & 2.50000000 \mathrm{sec}\end{array}$ SFO1 $500.3030896 \mathrm{MH}$ N1 11.20 usec LW1 F2 - Processing para $\begin{array}{ll}\text { SI } & 500.3000052 \mathrm{MHz} \\ \text { SF } & \end{array}$ WDW 


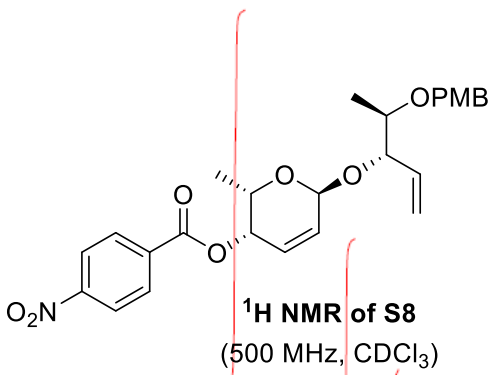

Current Data Parameters EXPNO

F2 - Acquisition Parameters $\begin{array}{rr}\text { Date_ } & 20190712 \\ \text { Time- } & 22.20\end{array}$

INSTRUM PROBHD $5 \mathrm{~mm}$ PABBO BB/
PULPROG

TD

SOLVENT

NS
DS

SWH $\quad 10000.000 \mathrm{~Hz}$

$\mathrm{AQ} \quad 3.2767999 \mathrm{sec}$ $\left.500 \mathrm{MHz}, \mathrm{CDCl}_{3}\right)$

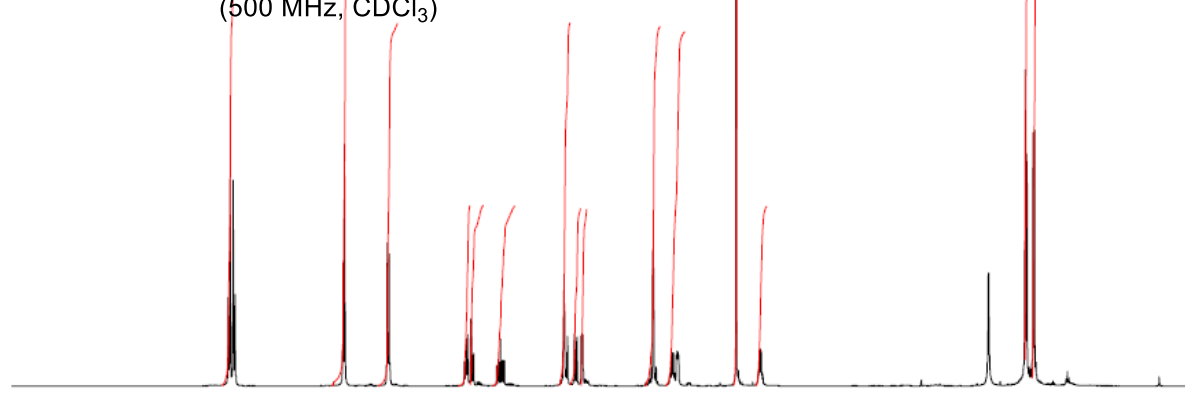

$\begin{array}{lr}\text { DW } & 50.000 \text { use } \\ \text { DE } & 6.50 \text { use }\end{array}$

$\begin{array}{lr}298.3 \mathrm{~K} \\ \mathrm{D} 1 & 2.00000000 \mathrm{sec}\end{array}$

SFO1 $500.3030896 \mathrm{MH}$

$\begin{array}{ll}\text { NUC1 } & 1 \mathrm{H} \\ \text { P1 } & 11.20 \text { usec }\end{array}$

F2 - Processing parameters

SI $\quad 500.3000136 \mathrm{MHz}$ SSB $\quad 0$

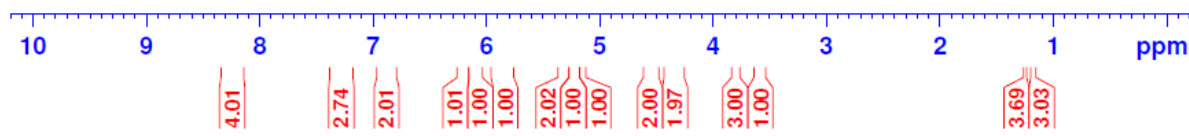

KJH-1-137-sam03 21320190923

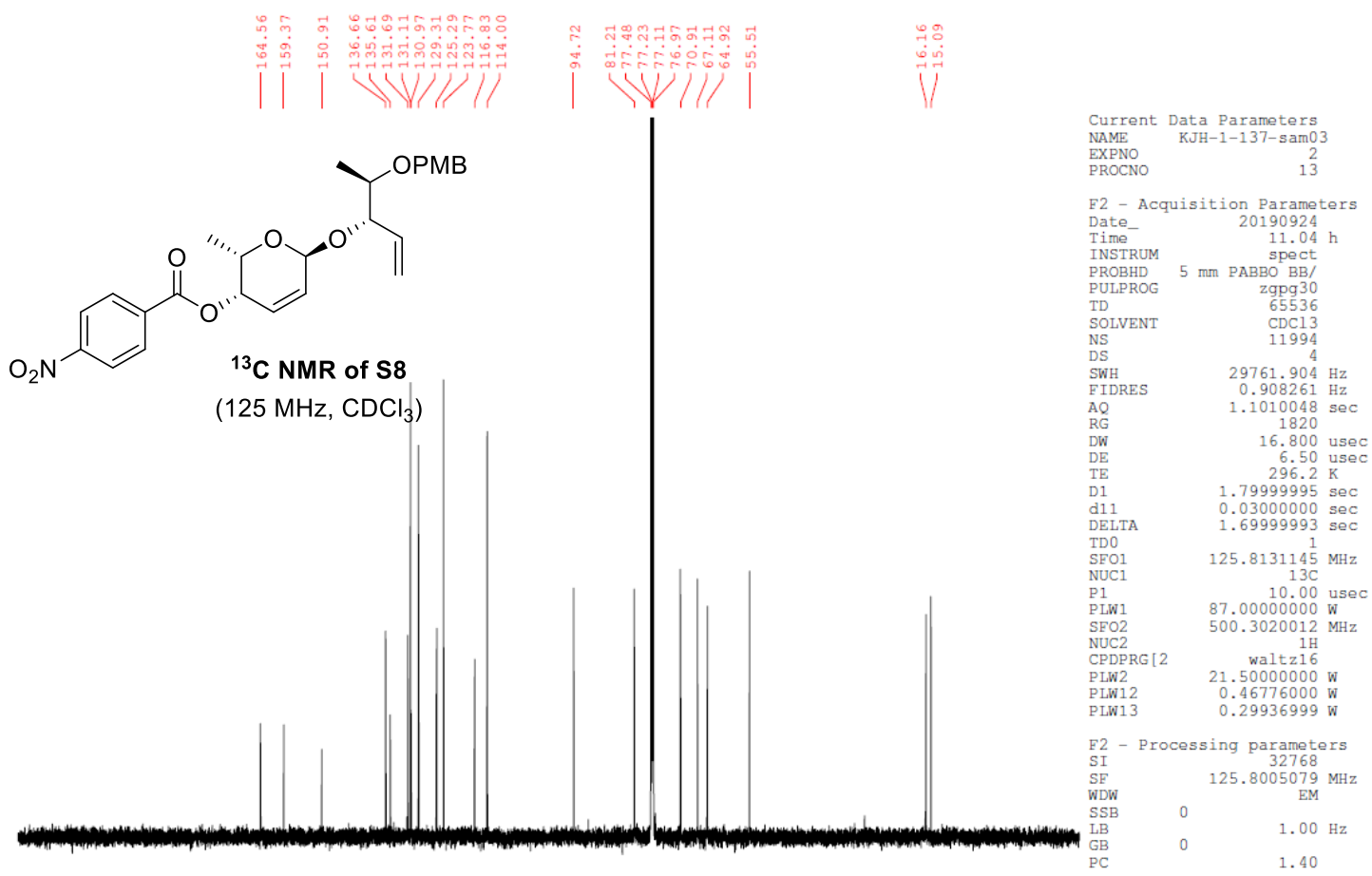

$\begin{array}{llllllllllll}200 & 180 & 160 & 140 & 120 & 100 & 80 & 60 & 40 & 20 & 0 & \mathrm{ppm}\end{array}$

S33 

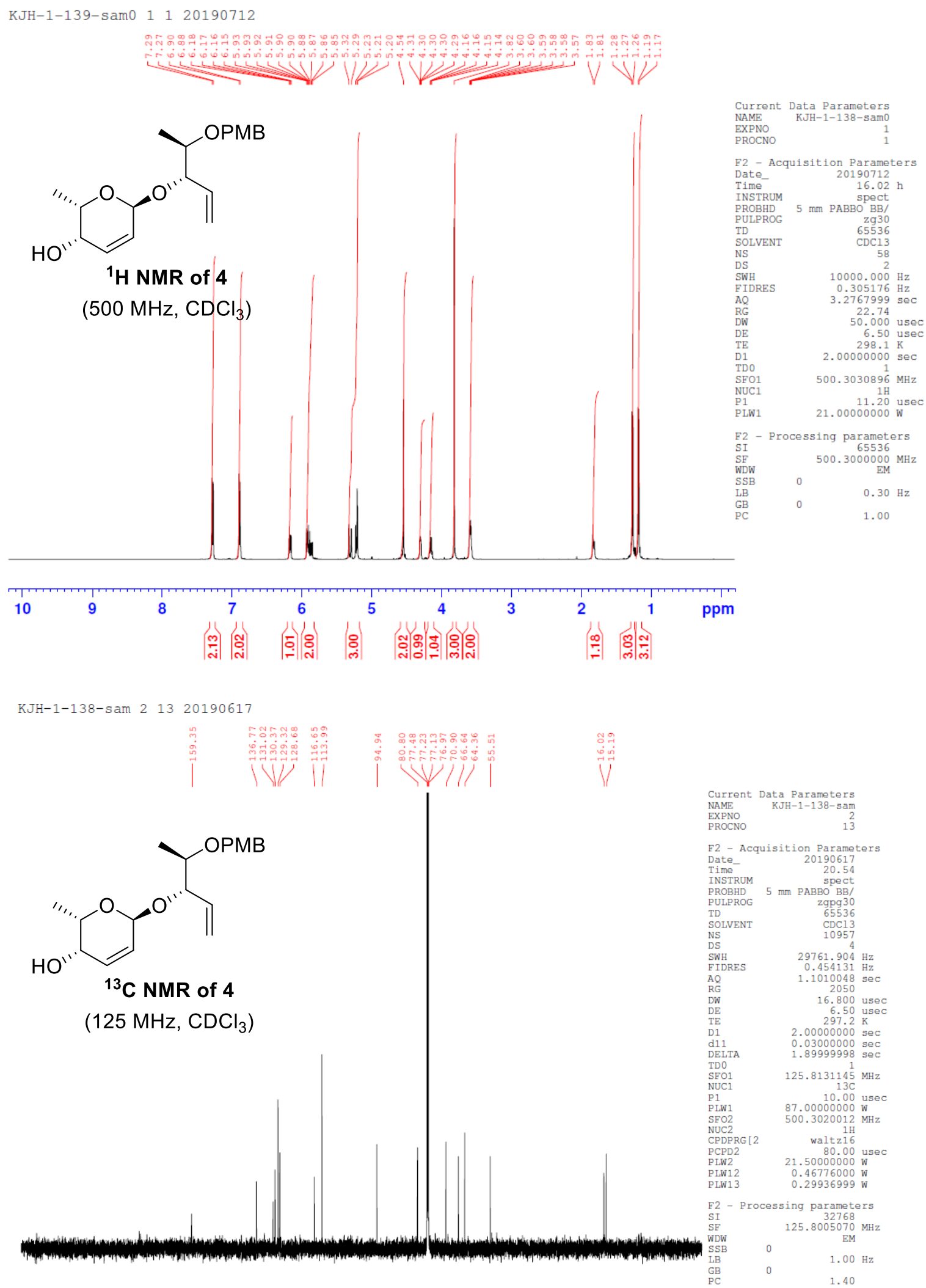

$\begin{array}{llllllllllll}200 & 180 & 160 & 140 & 120 & 100 & 80 & 60 & 40 & 20 & 0 & \mathrm{ppm}\end{array}$ 


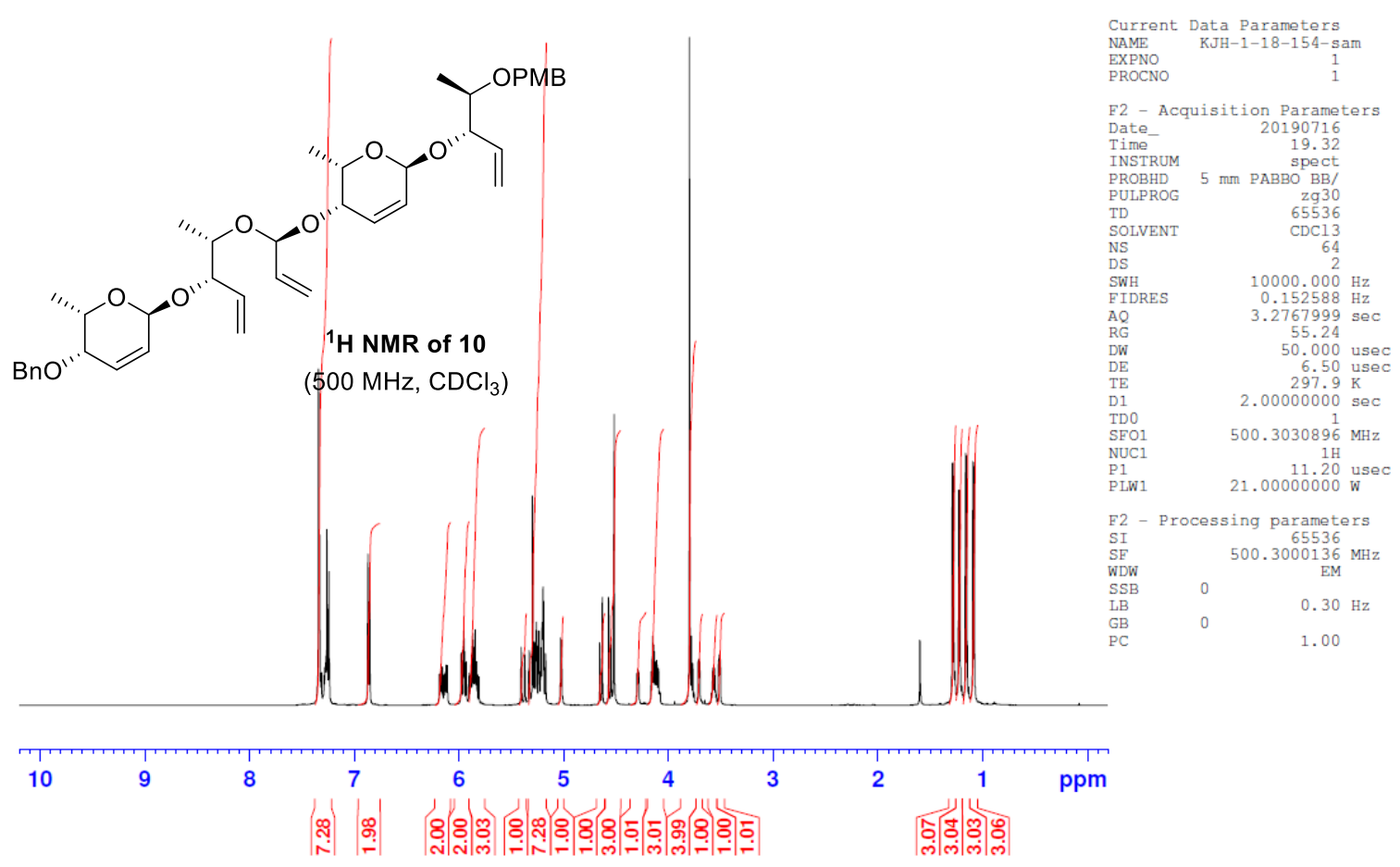

KJH-1-154-sam 31320190715

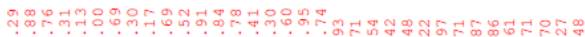

月.

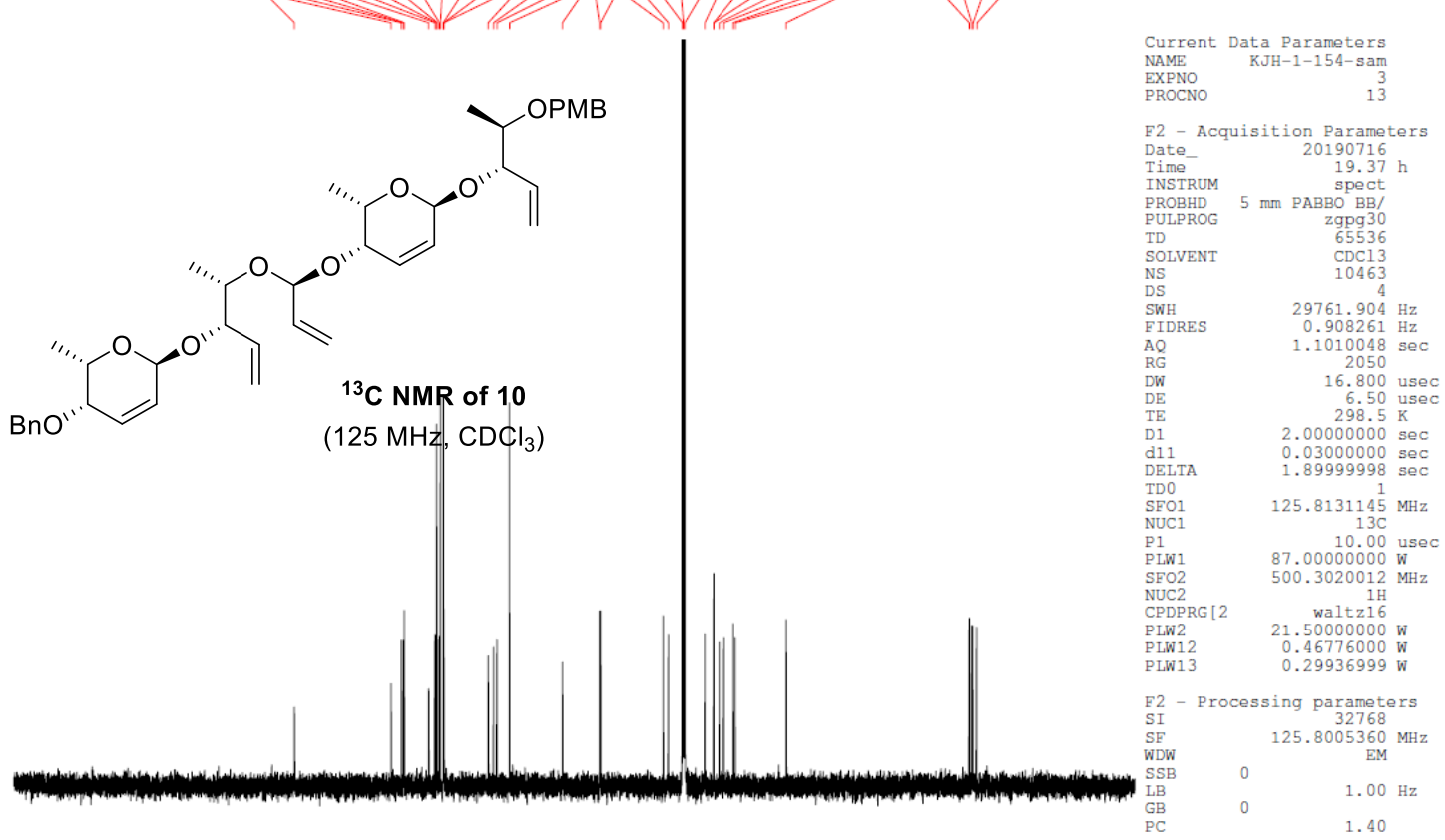

$200 \quad 180 \quad 160$

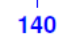

120

100

80

60

20

0 ppm 

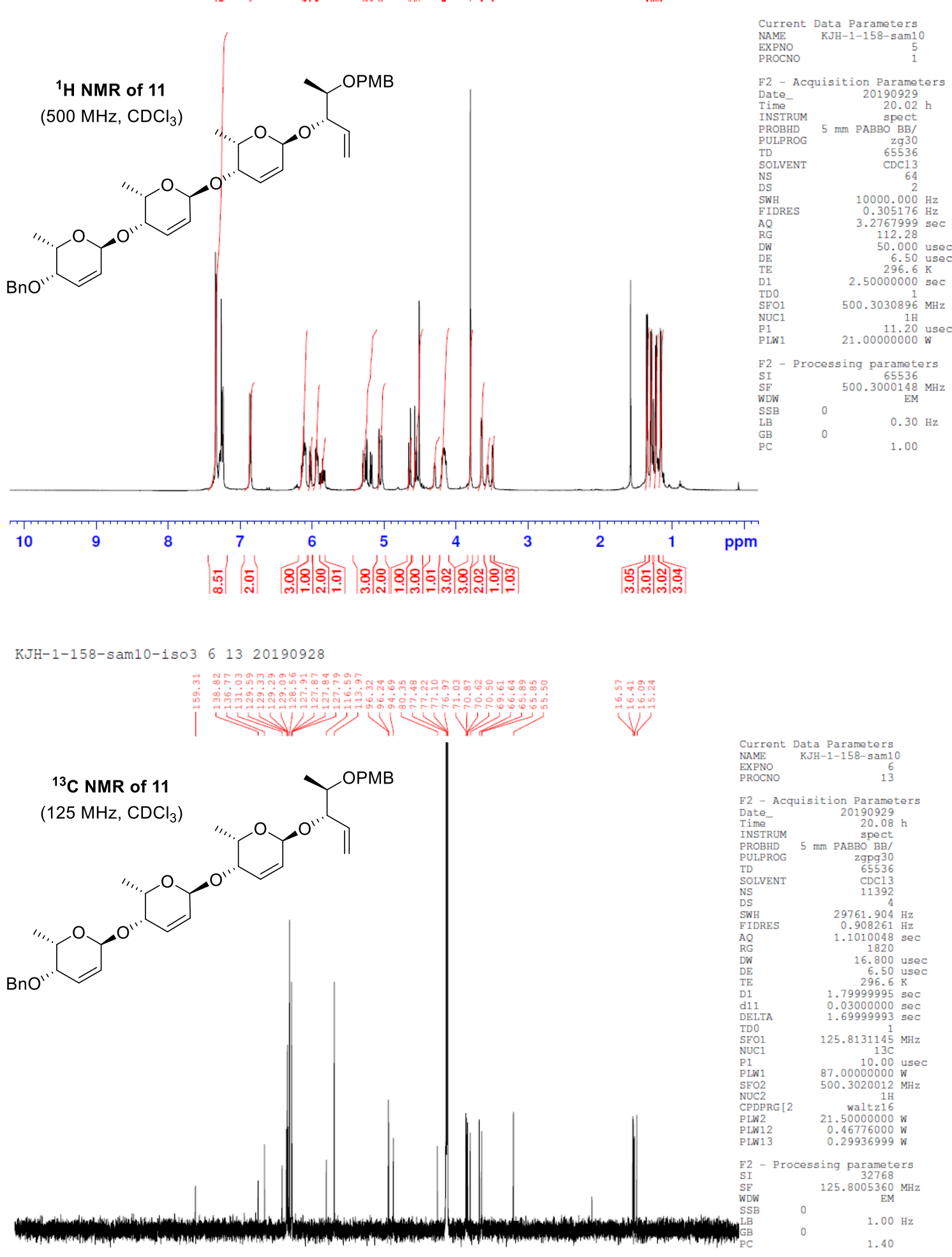

$\begin{array}{llllllllllll}200 & 180 & 160 & 140 & 120 & 100 & 80 & 60 & 40 & 20 & 0 & \mathrm{ppm}\end{array}$ 


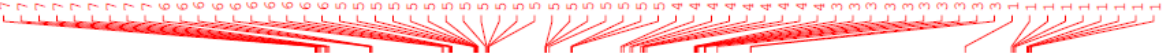

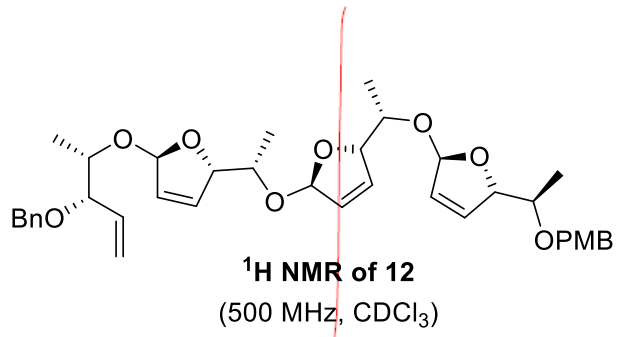

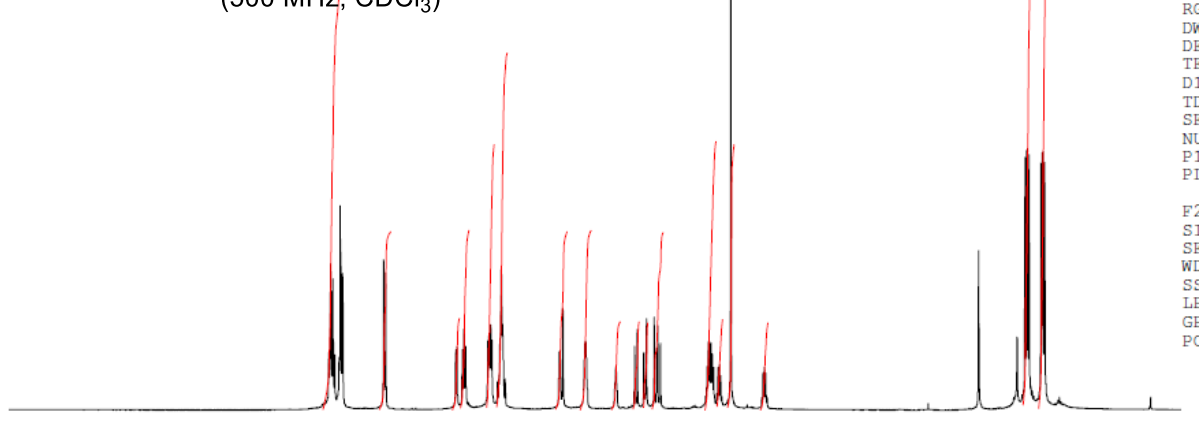

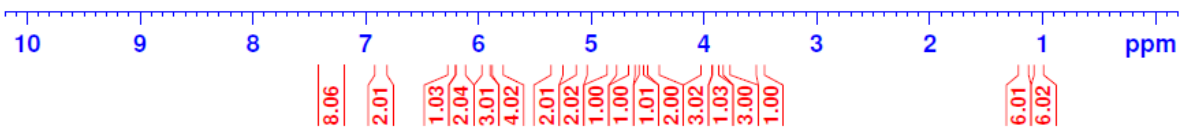

KJH-1-158-sam10-iso1 21320190928

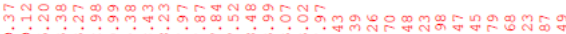

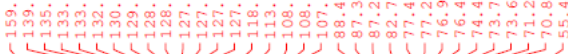

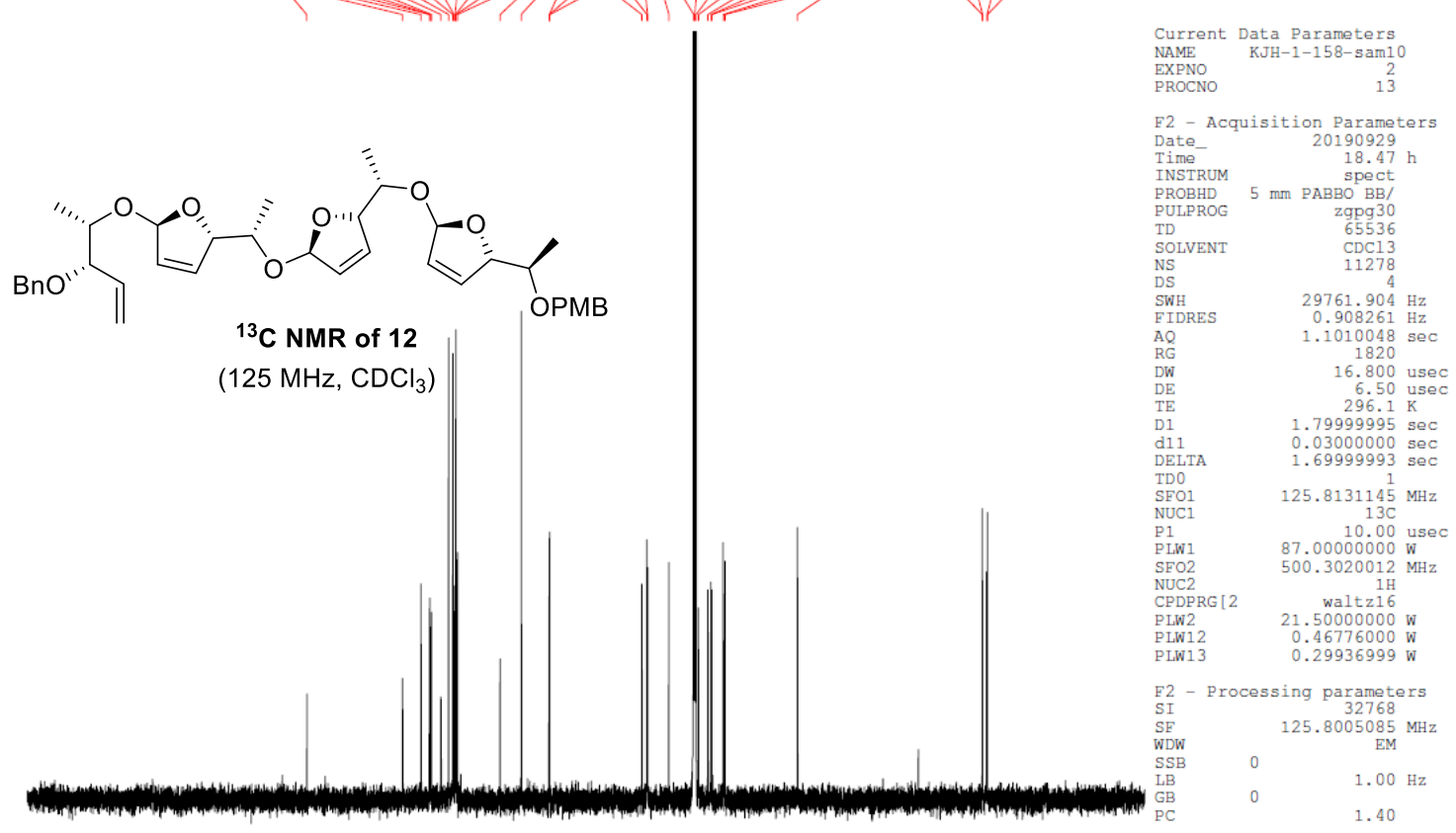

Current Data Parameters

NAME
EXPNO

F2 - Acquisition Paraneters

F2 - Acquisition Paramete
Date_ 20190929

$\begin{array}{ll}{ }_{\text {Time }} & 18.41 \\ \text { INSTRUM } & \text { spect }\end{array}$

PROBHD $5 \mathrm{~mm}$ PABBO BB/

$\begin{array}{ll}\text { TD } & 65536 \\ \text { SOLVENT } & \text { CDC13 }\end{array}$

DS $\quad 10000.000 \mathrm{~Hz}$

$\quad r 6$
$R$ TW
50.000 usec

6.50 usec
$296.2 \mathrm{~K}$
2.50000000

1
se

$500.3030896 \mathrm{MHz}$
NUC1

$\begin{array}{ll}1 & 11.20 \\ & 21.00000000 \text { W }\end{array}$

2 - Processing parameters

$\begin{array}{lc}\text { SI } & 65536 \\ \text { SF } & 500.3000141 \mathrm{MHz} \\ \text { WDW } & \text { EM M }\end{array}$

$\begin{array}{lll}\text { SSB } & 0 & 0.30 \mathrm{~Hz}\end{array}$ $\begin{array}{ll}0.305176 \mathrm{~Hz} \\ \mathrm{H} & 3.2767999 \mathrm{sec}\end{array}$

m

1.00

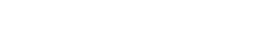




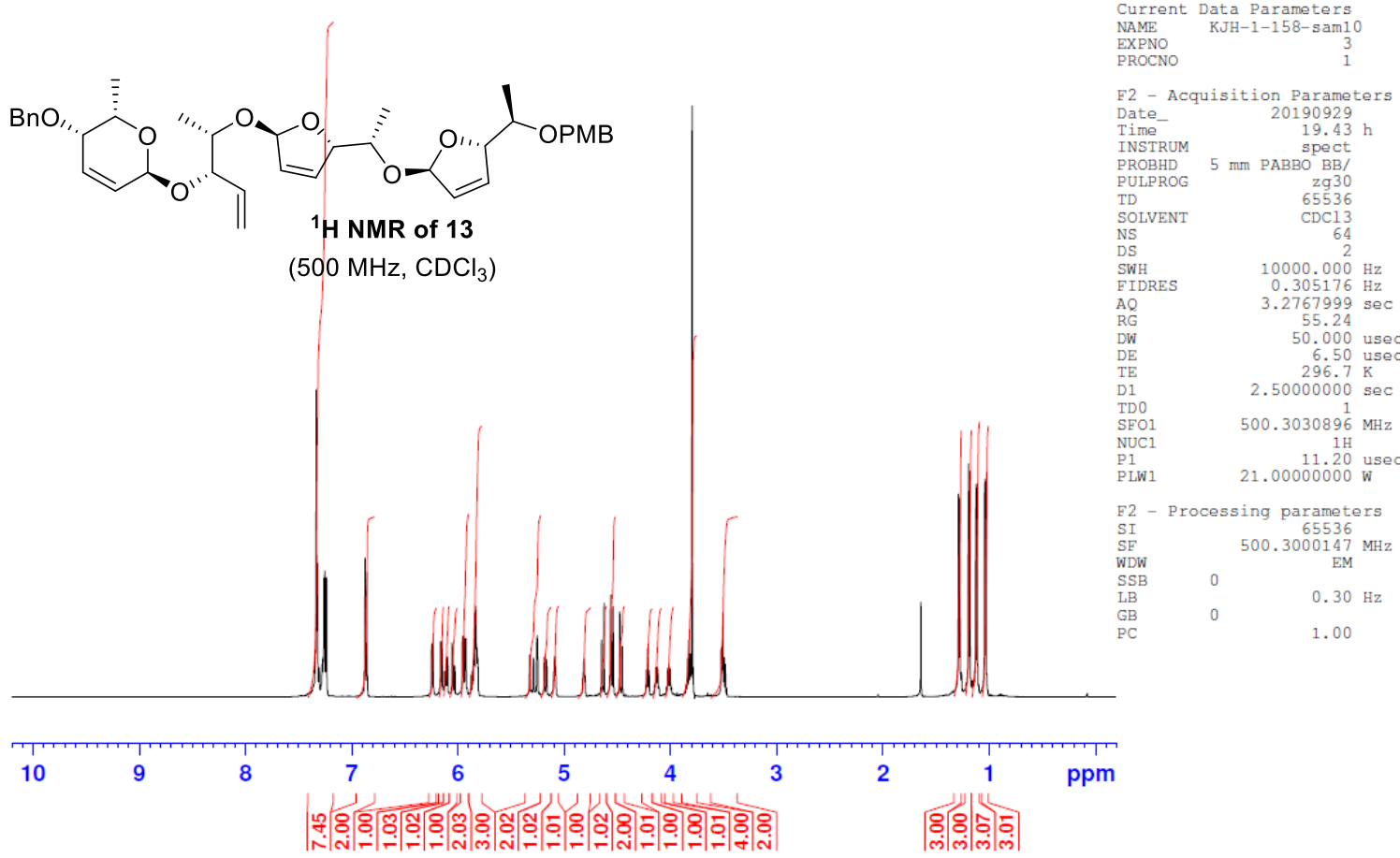

KJH-1-158-sam10-iso2 41320190928

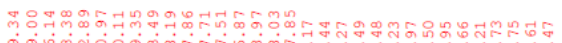

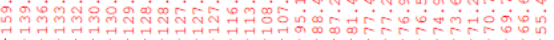

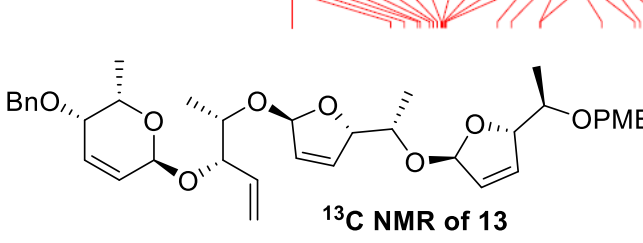

$\left(125 \mathrm{MHz}, \mathrm{CDCl}_{3}\right)$
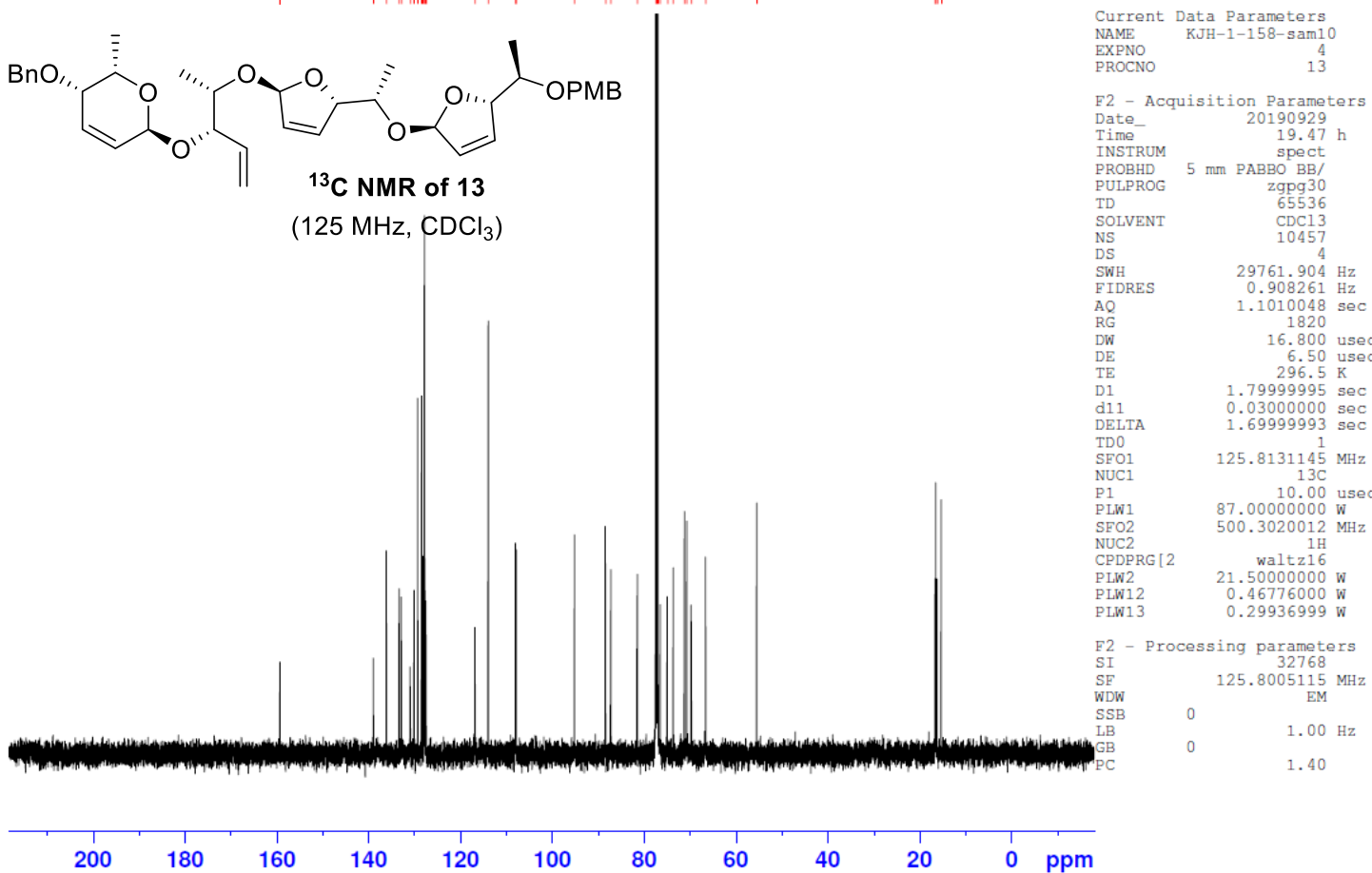


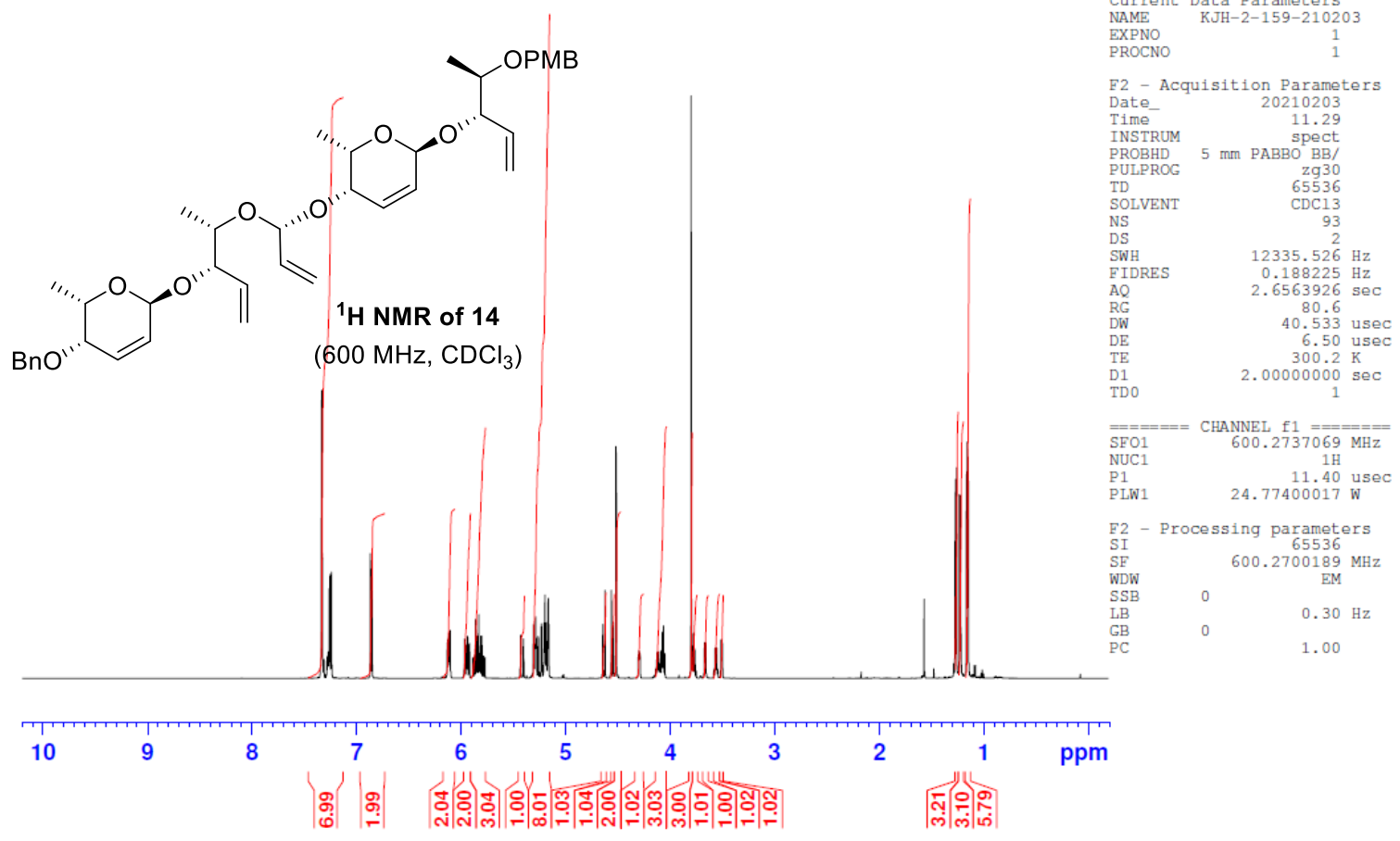

KJH-2-159-210203

$13 \mathrm{C}$

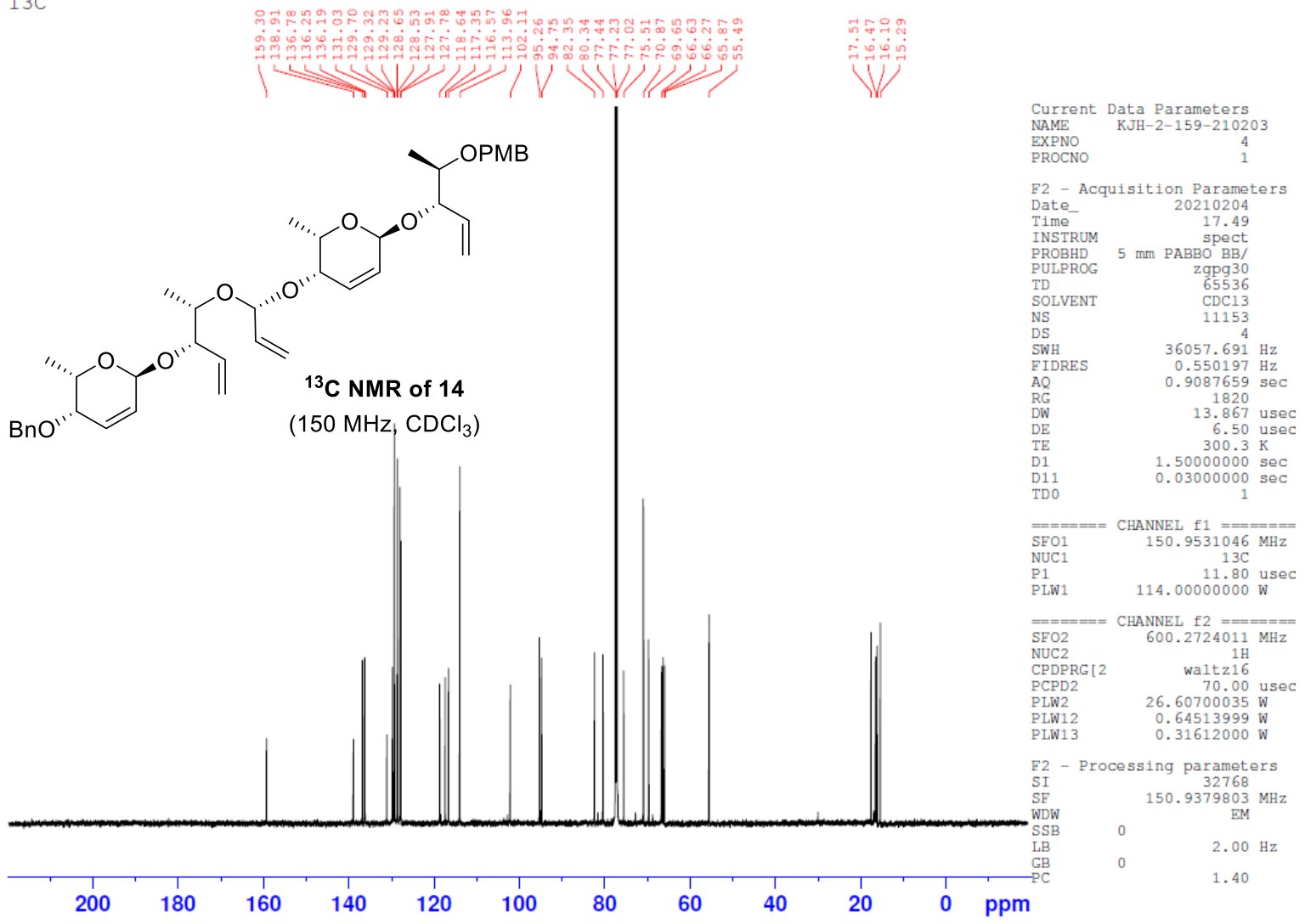



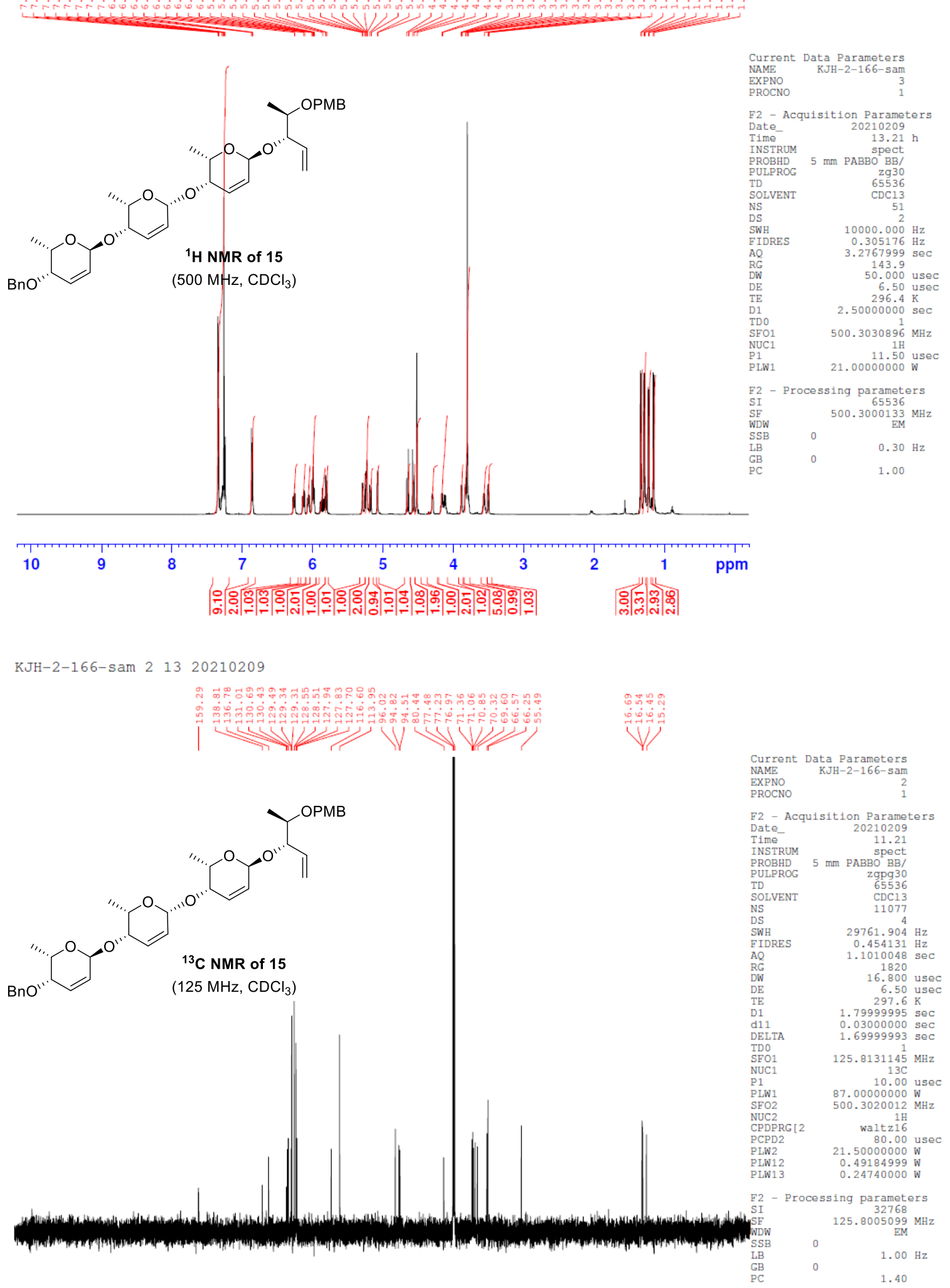

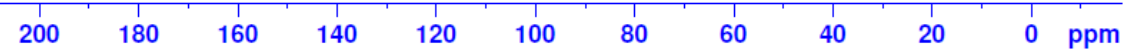




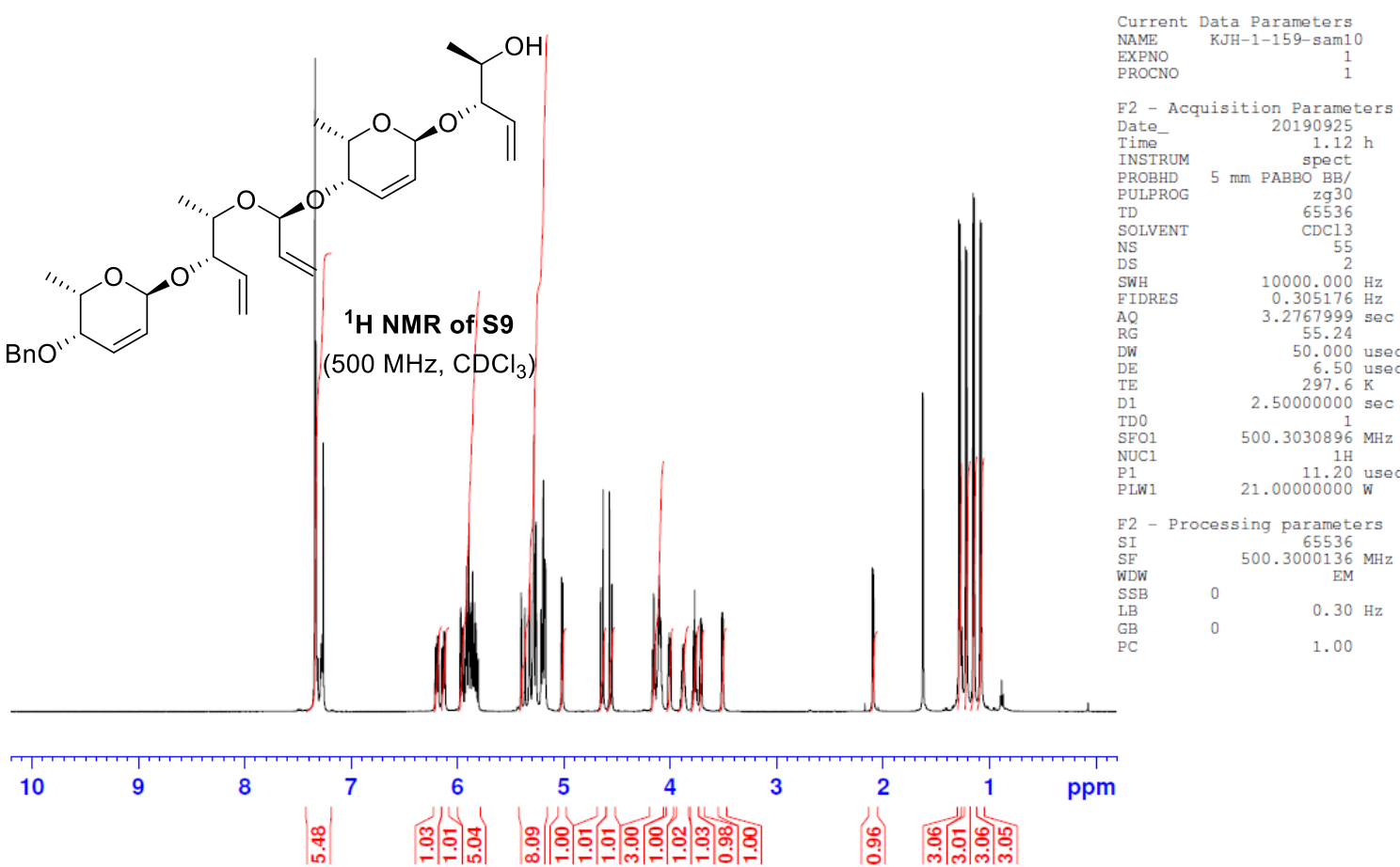

KJH-1-159-sam 21320190925

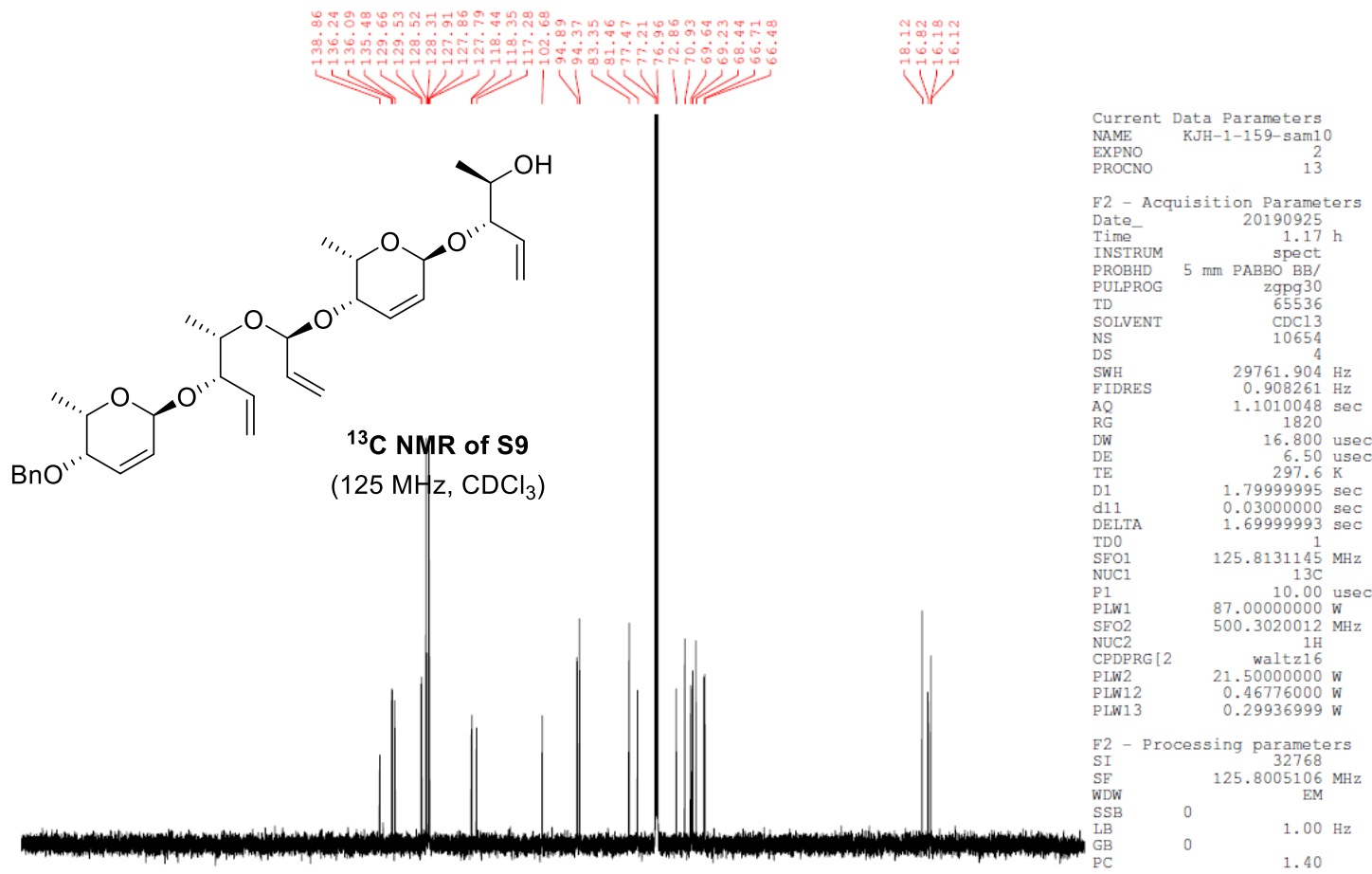

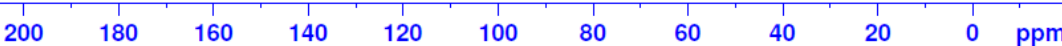




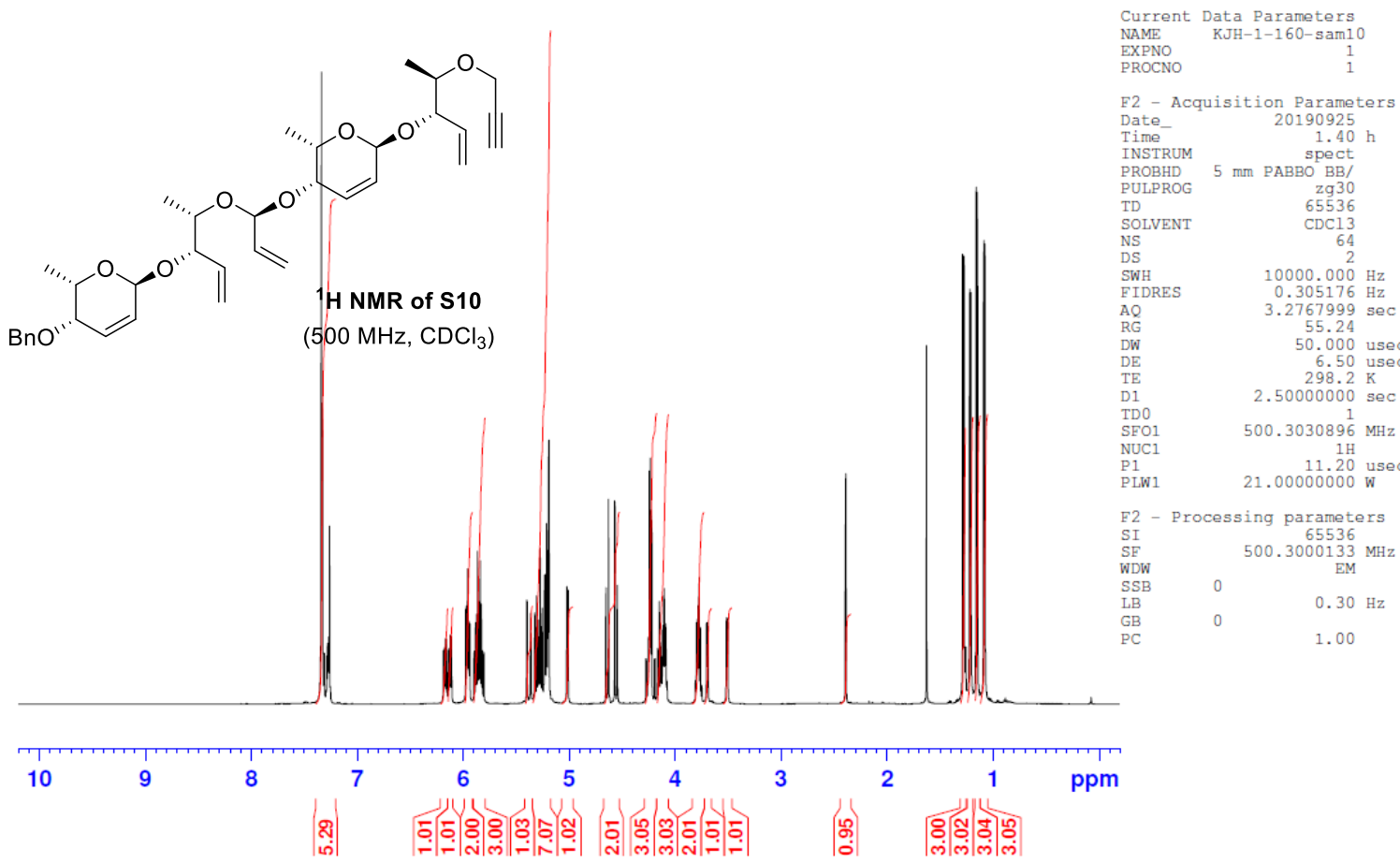

KJH-1-160-sam10 21320190925

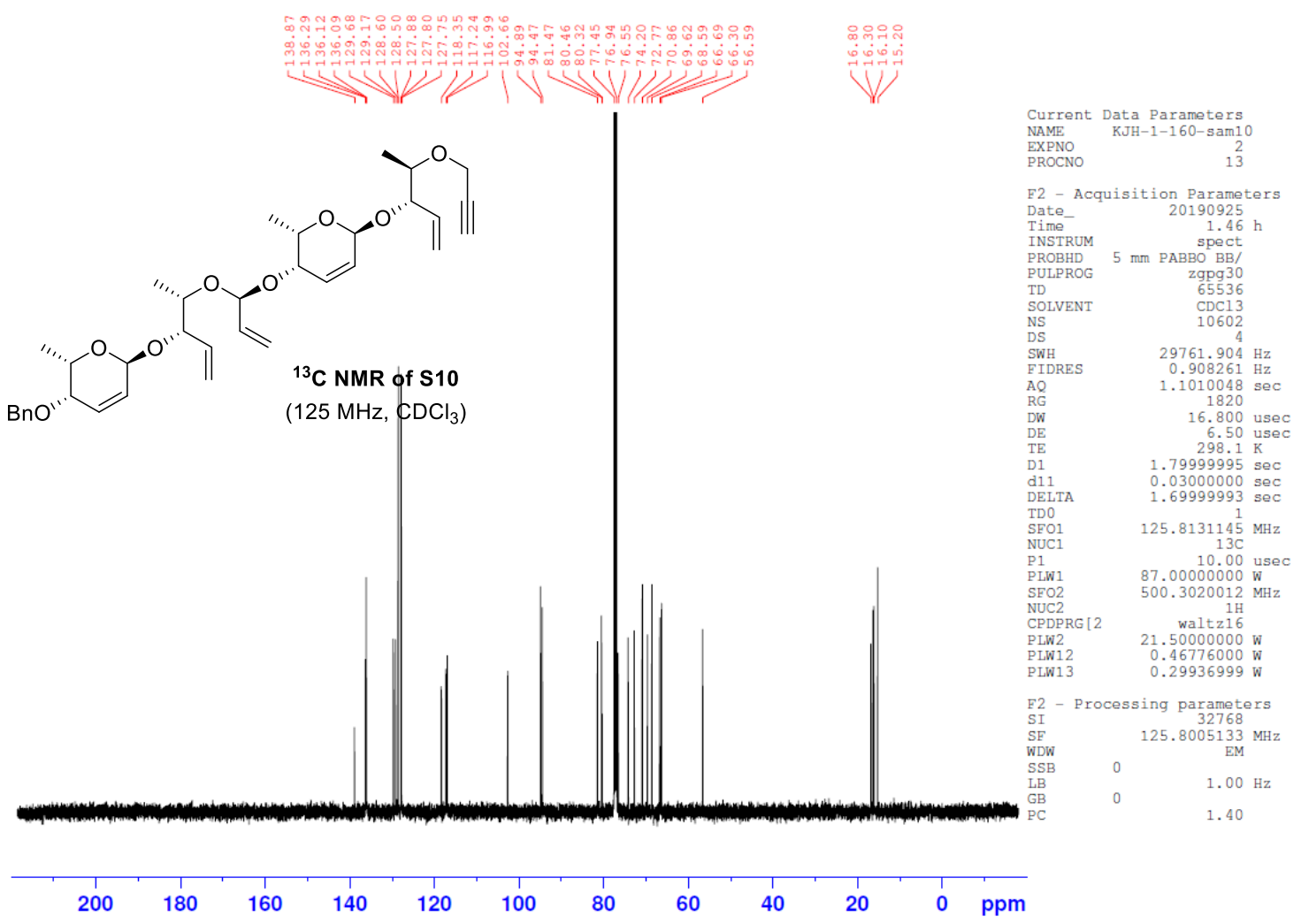



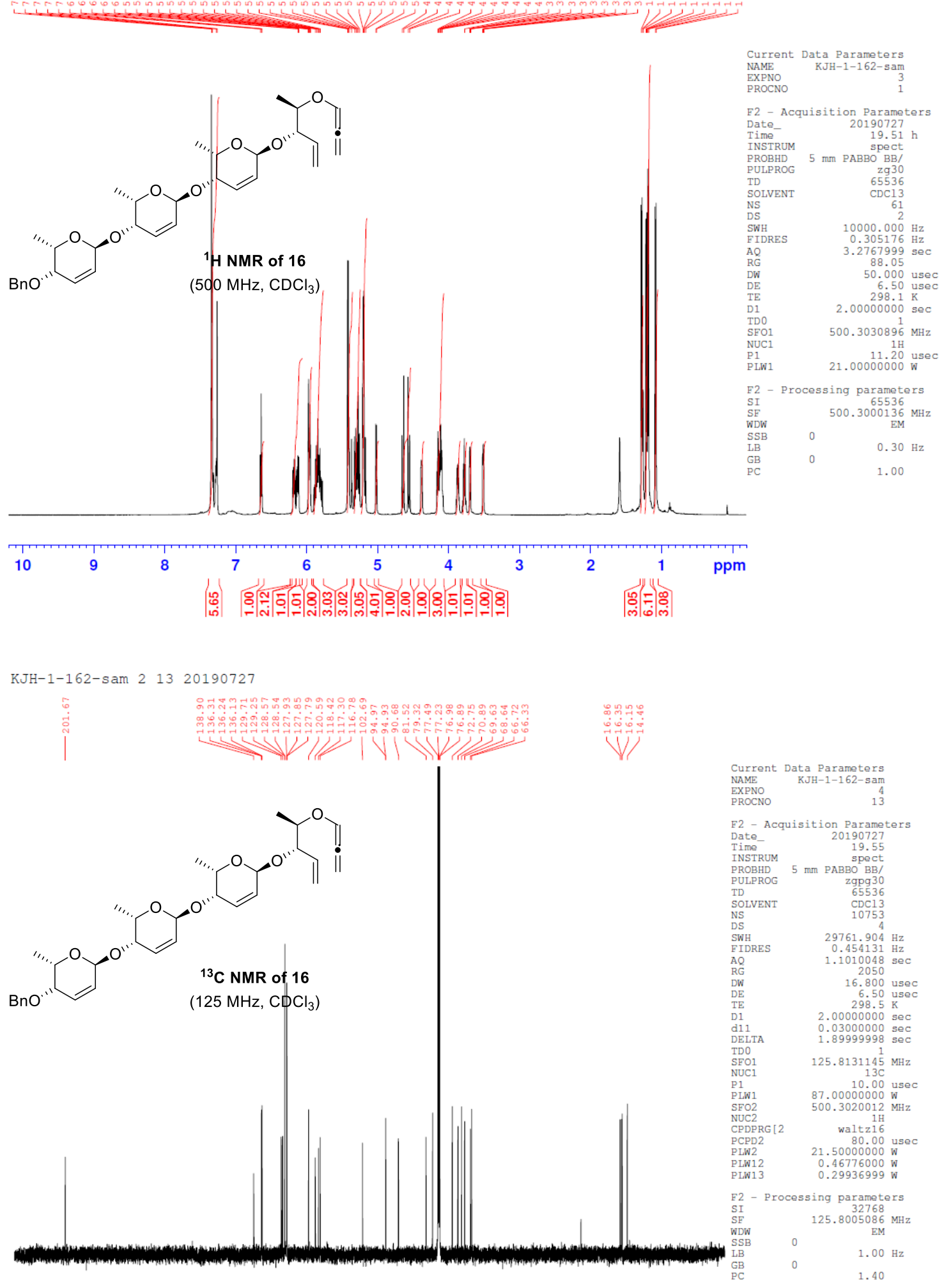

$\begin{array}{llllllllllll}200 & 180 & 160 & 140 & 120 & 100 & 80 & 60 & 40 & 20 & 0 & \mathrm{ppm}\end{array}$ 

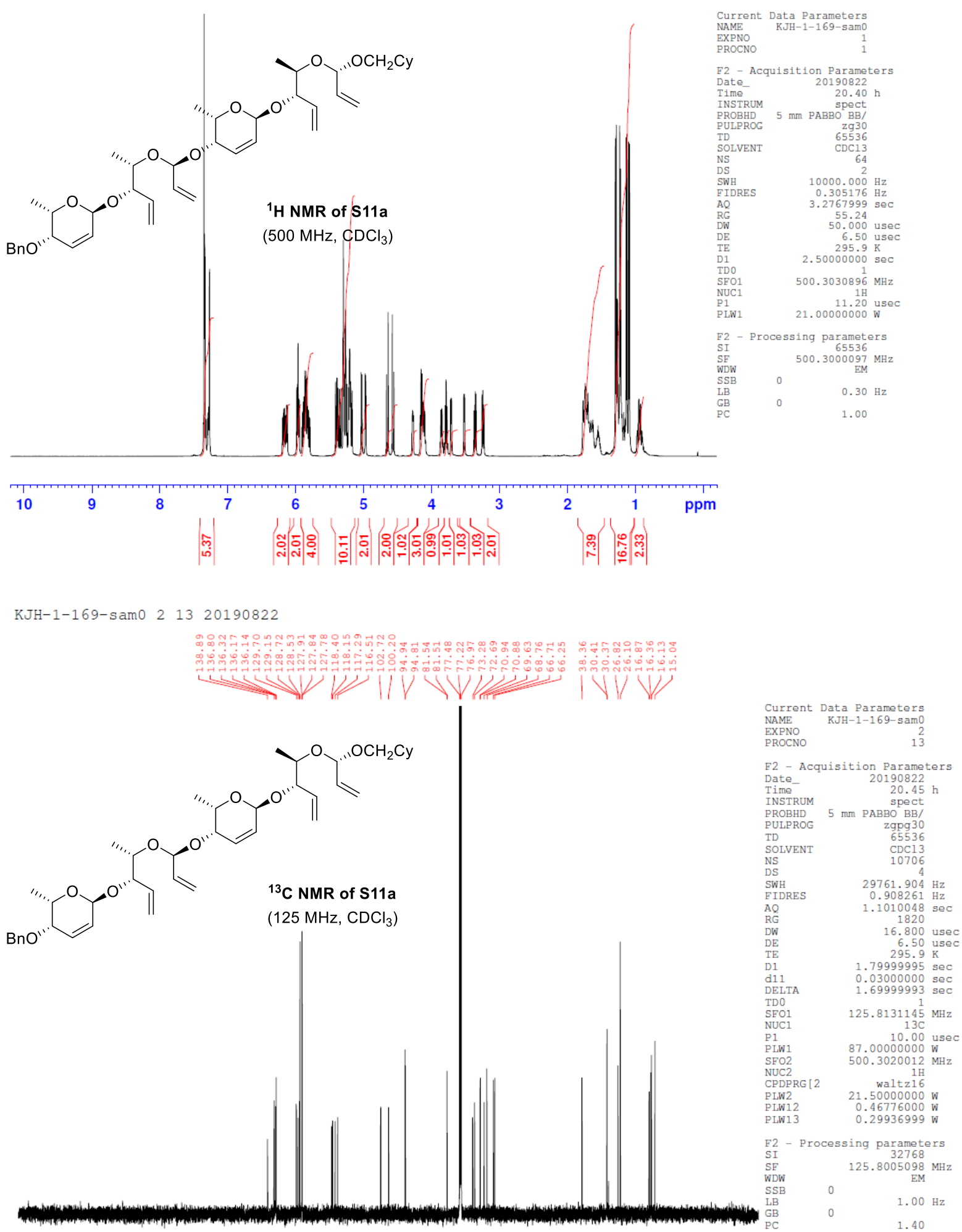

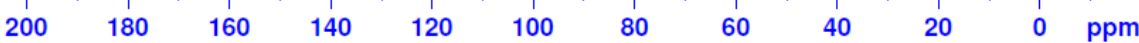




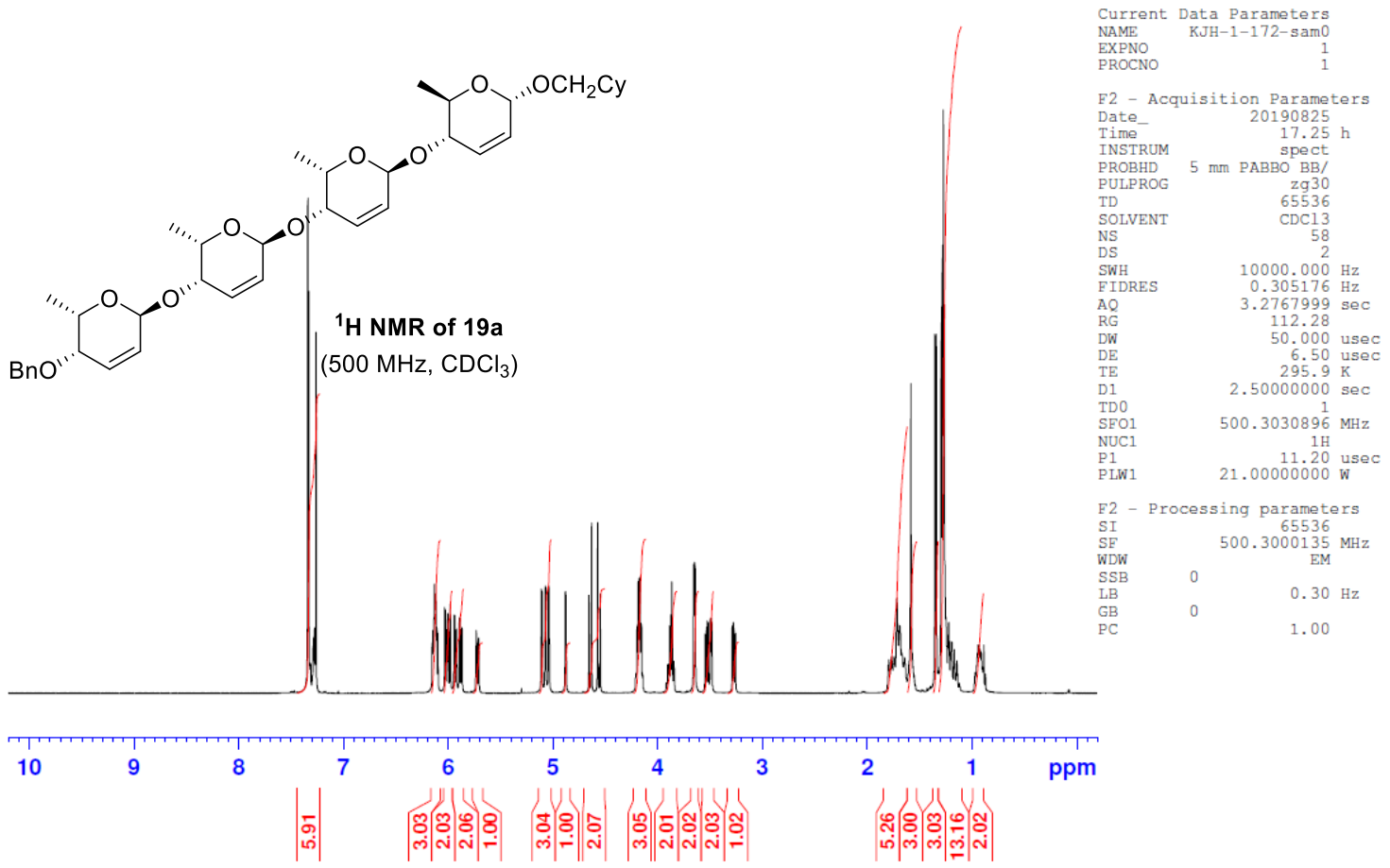

KJH-1-172-sam0 21320190825

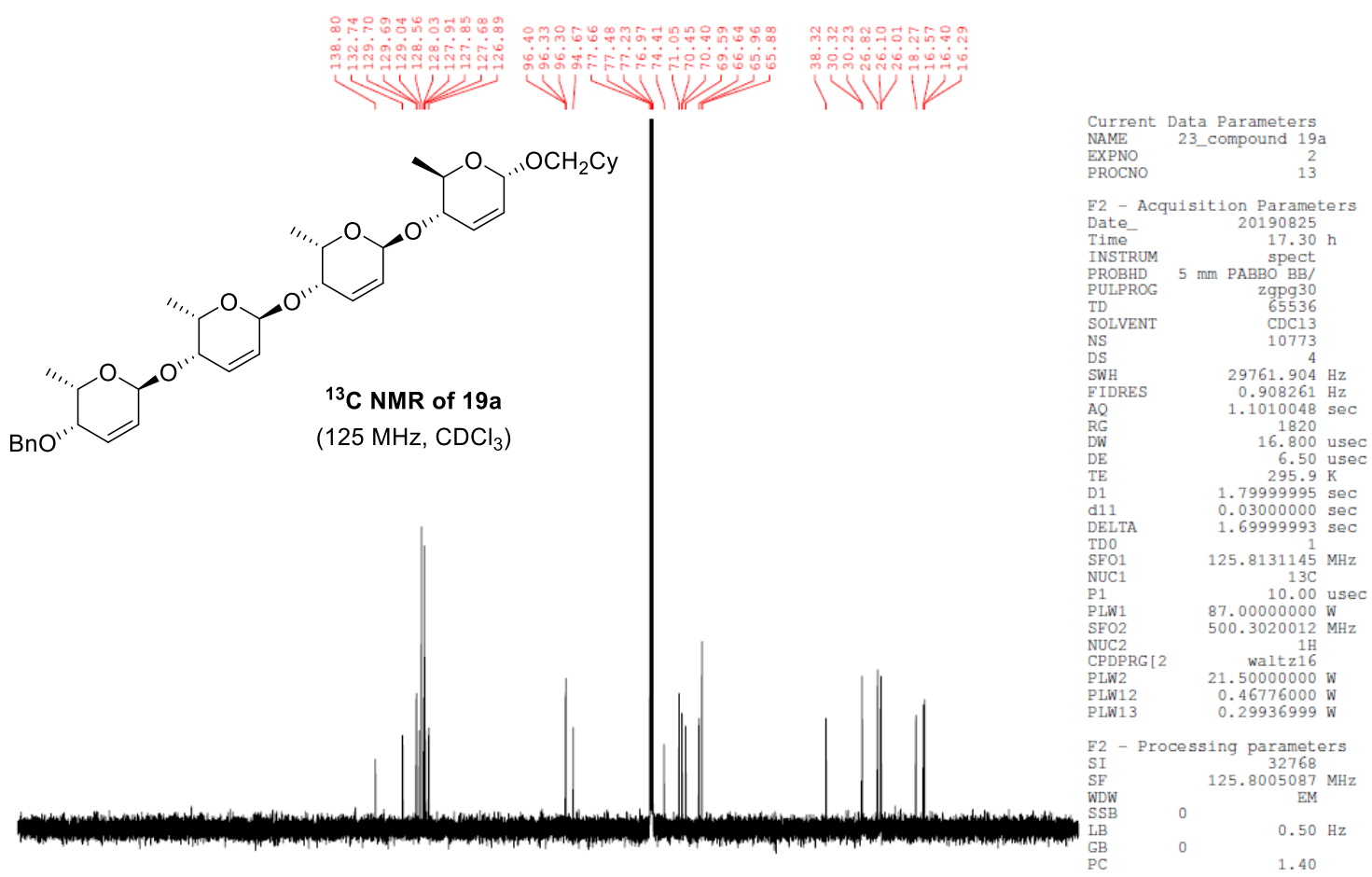



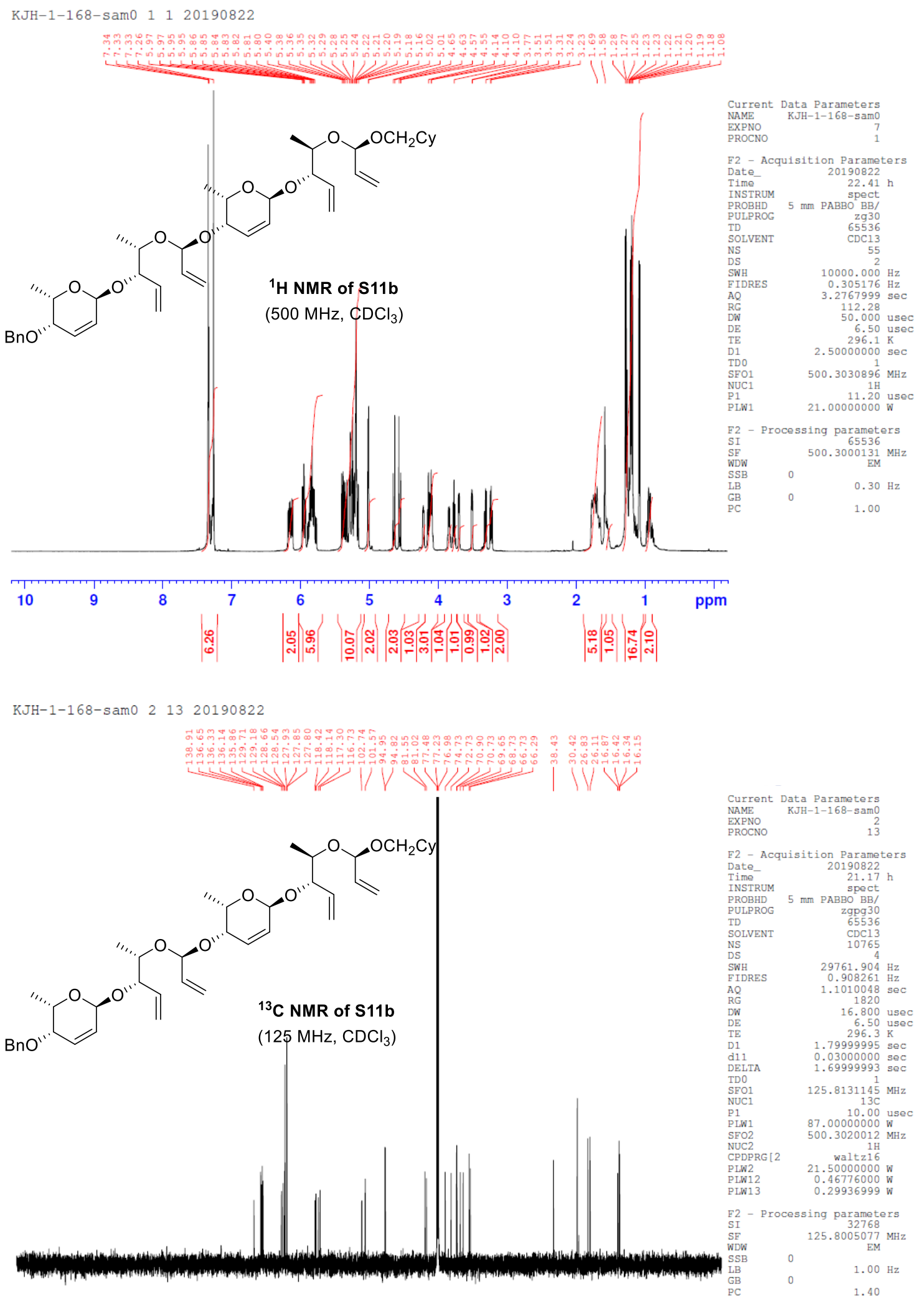

$\begin{array}{llllllllllll}200 & 180 & 160 & 140 & 120 & 100 & 80 & 60 & 40 & 20 & 0 & \mathrm{ppm}\end{array}$ 


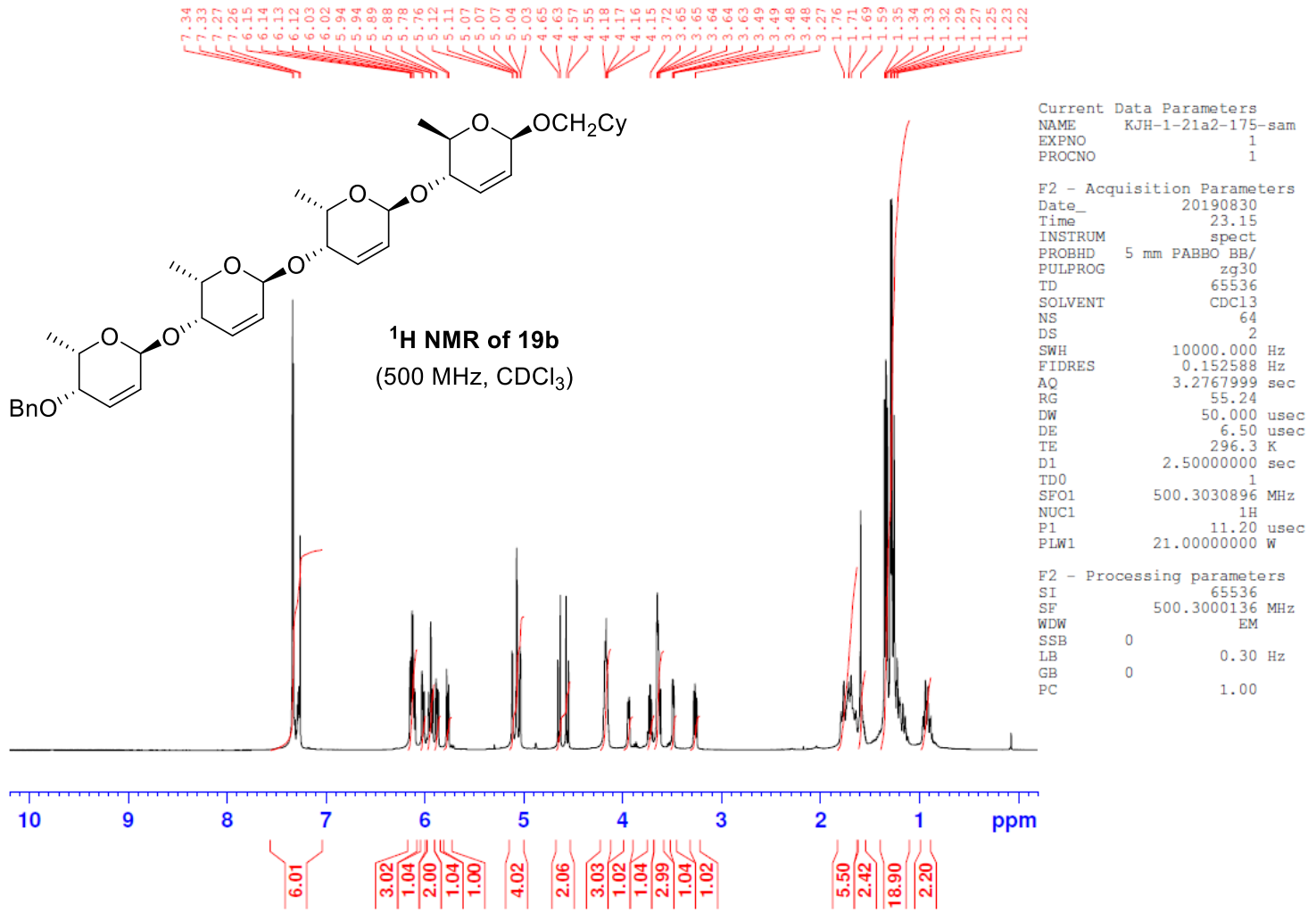

KJH-1-175-sam 21320190829 CH2CL2

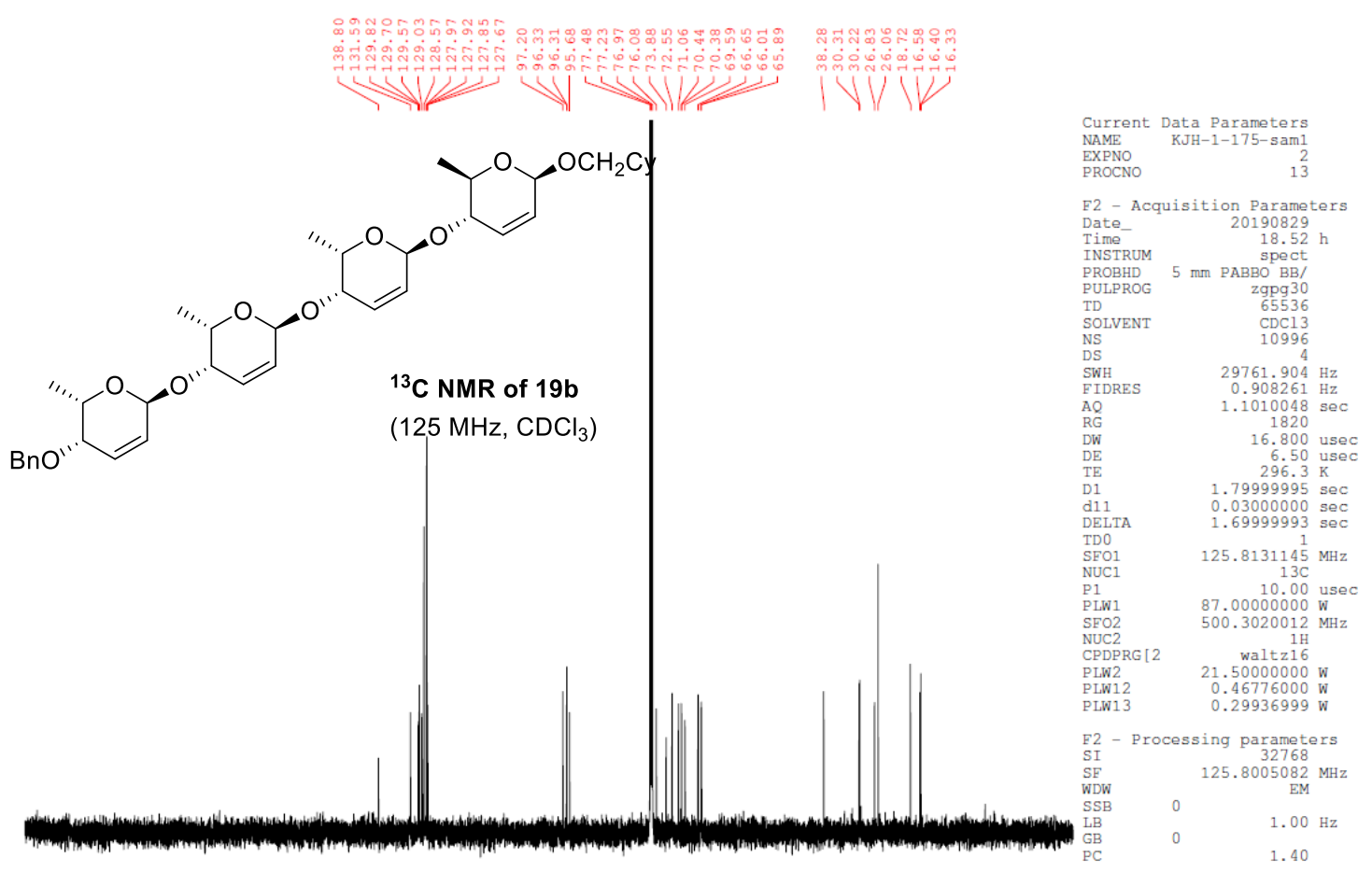

$\begin{array}{llllllllllll}200 & 180 & 160 & 140 & 120 & 100 & 80 & 60 & 40 & 20 & 0 & \mathrm{ppm}\end{array}$ 

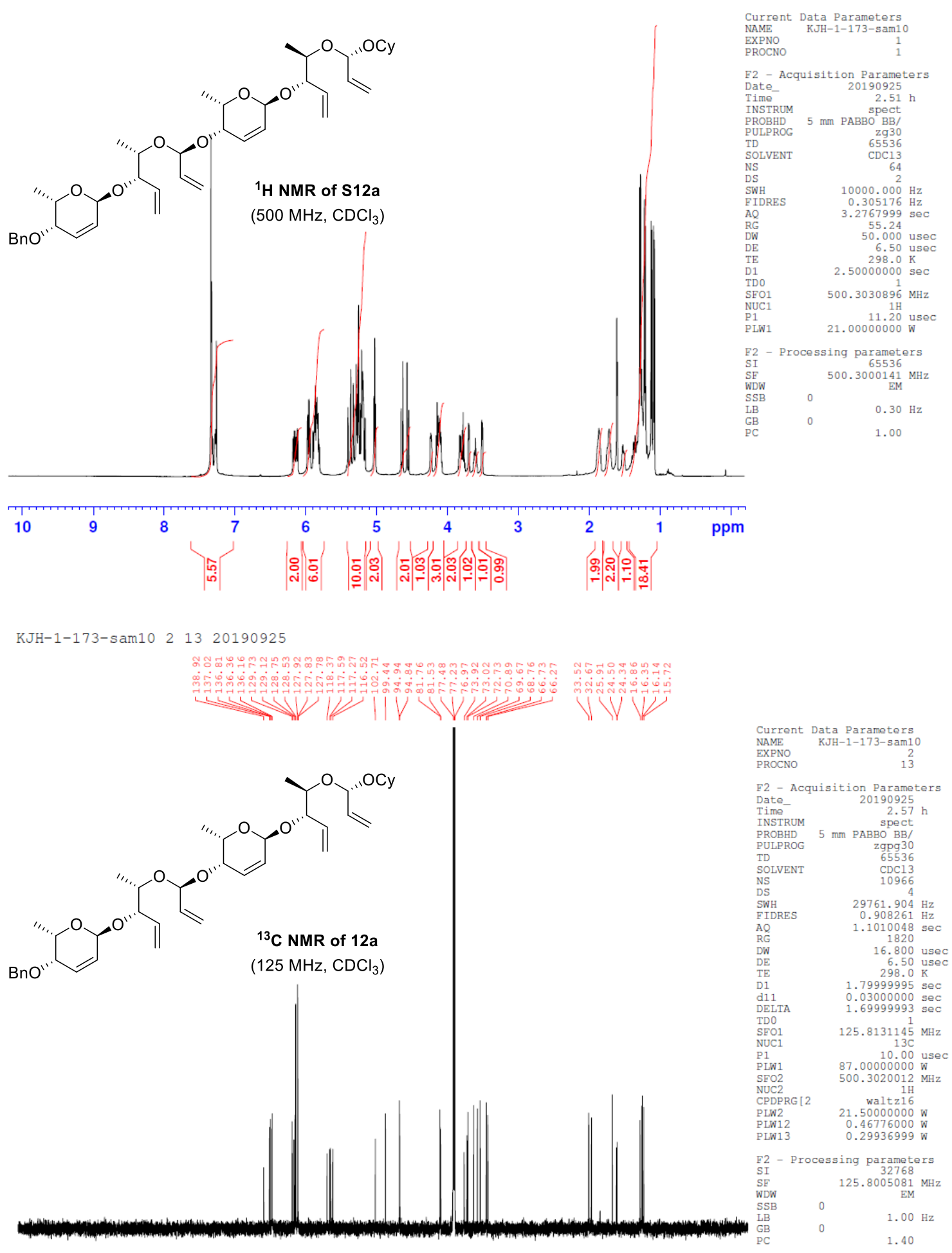

$\begin{array}{llllllllllll}200 & 180 & 160 & 140 & 120 & 100 & 80 & 60 & 40 & 20 & 0 & \text { ppm }\end{array}$ 

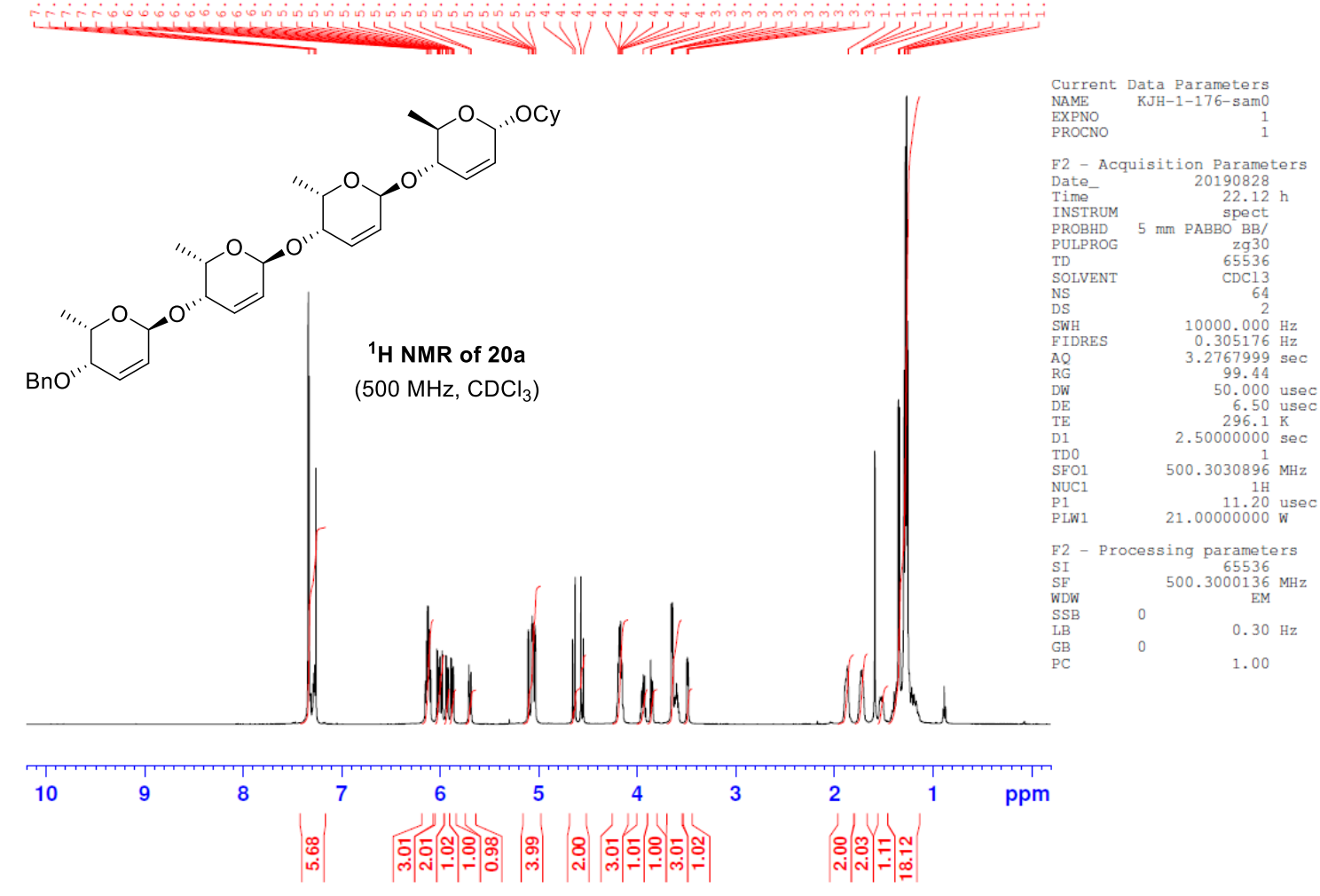

KJH-1-176-sam0 21320190828

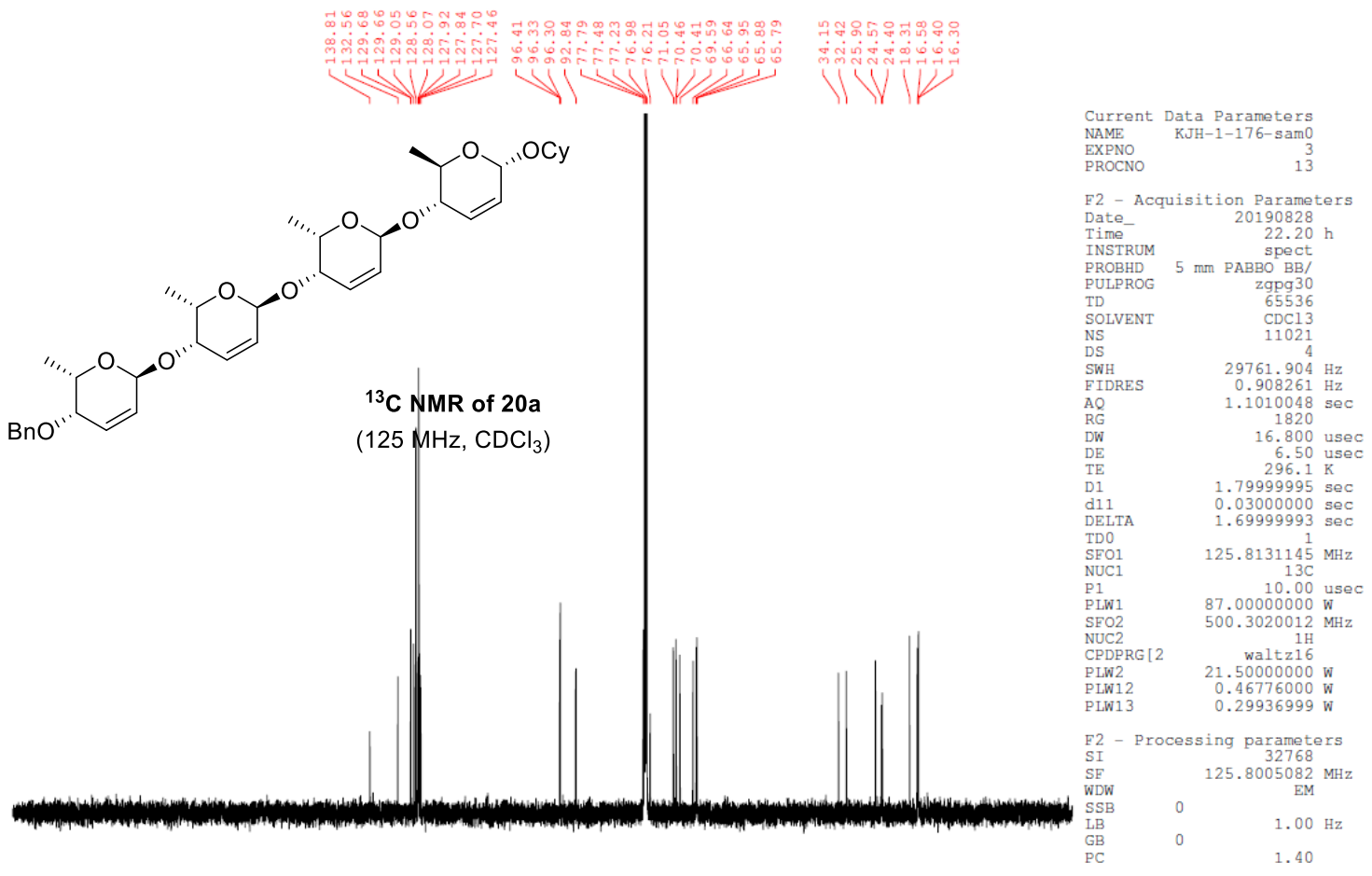




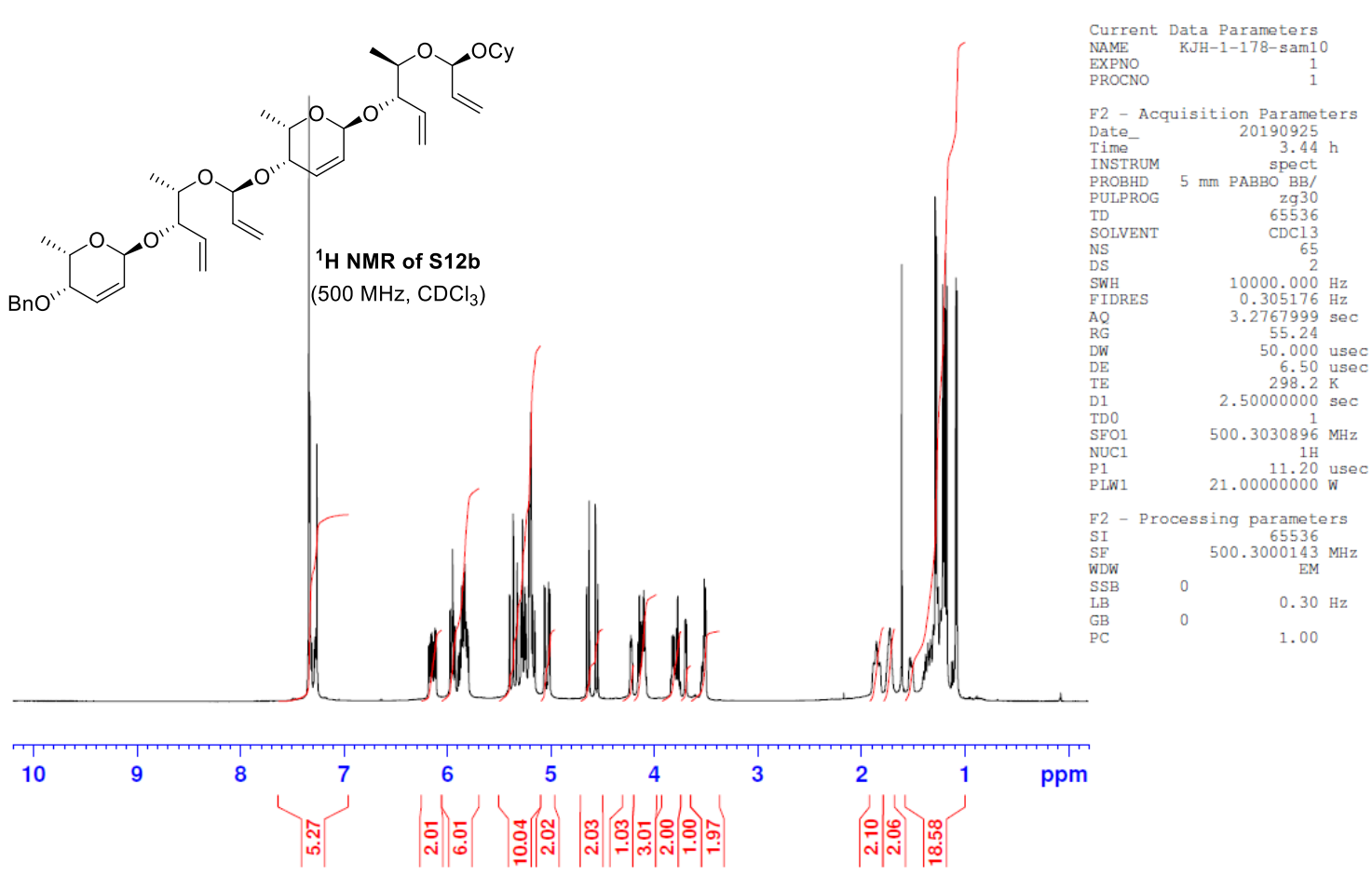

KJH-1-178-sam1 21320190925

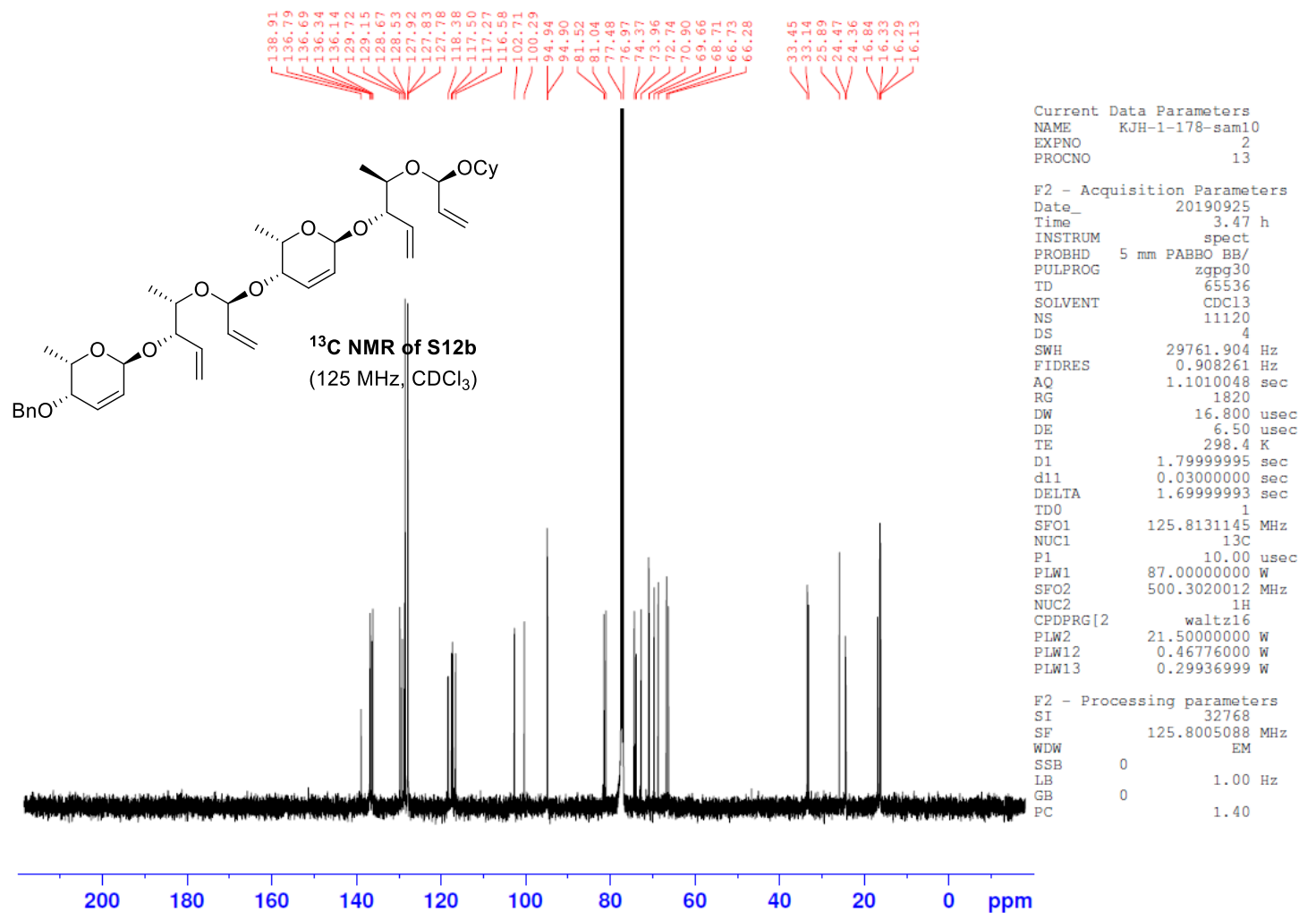




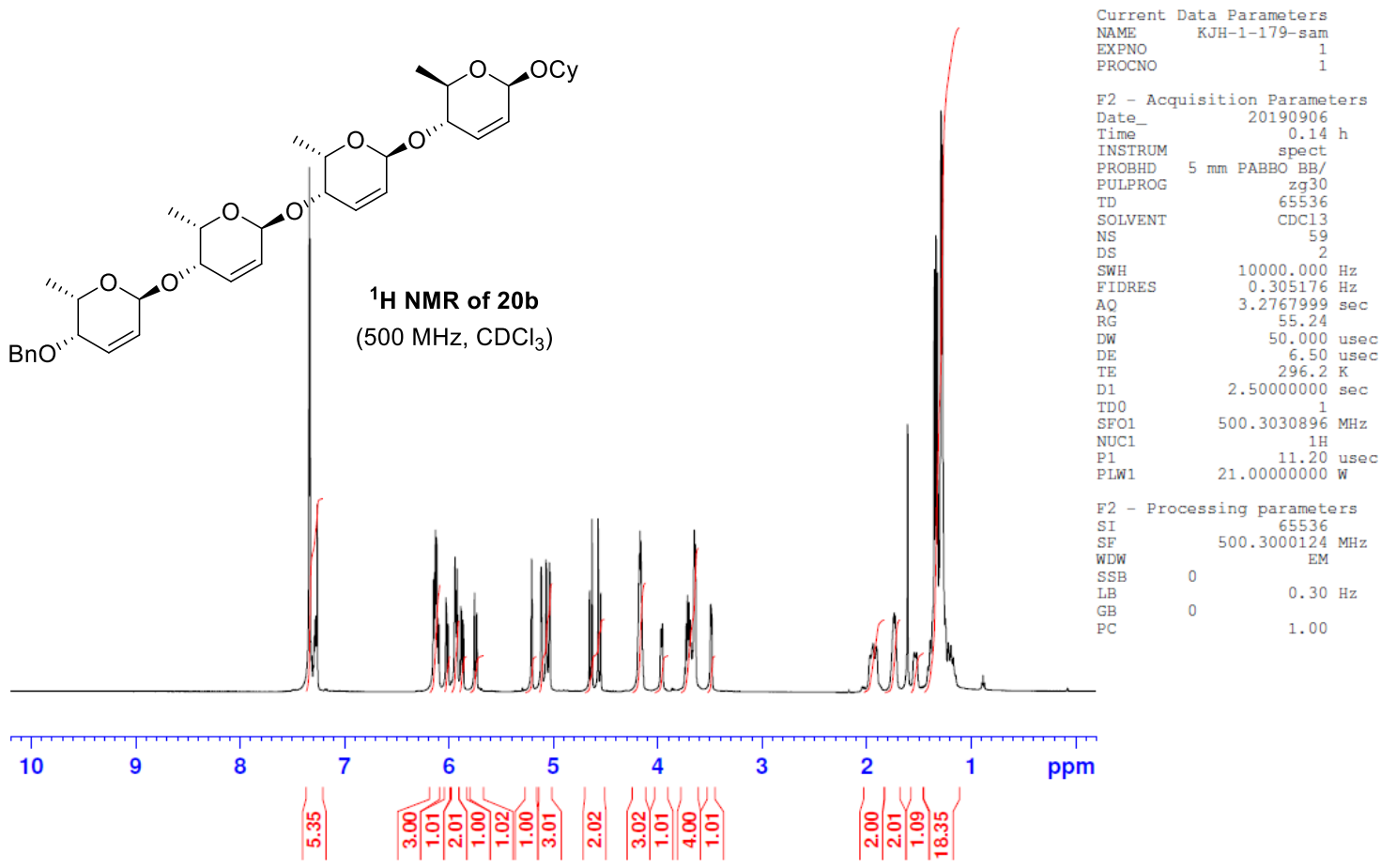

KJH-1-179-sam 21320190905
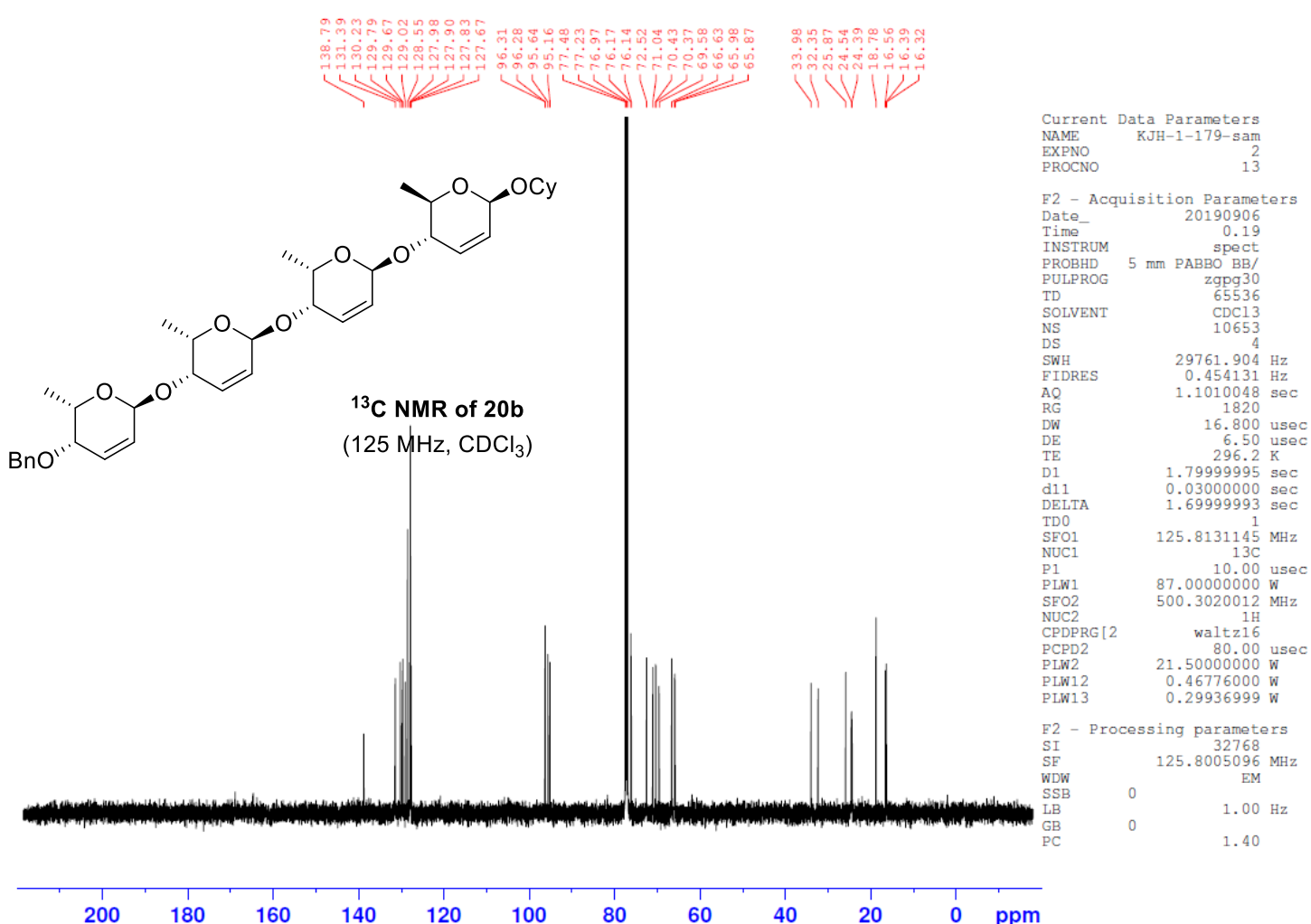


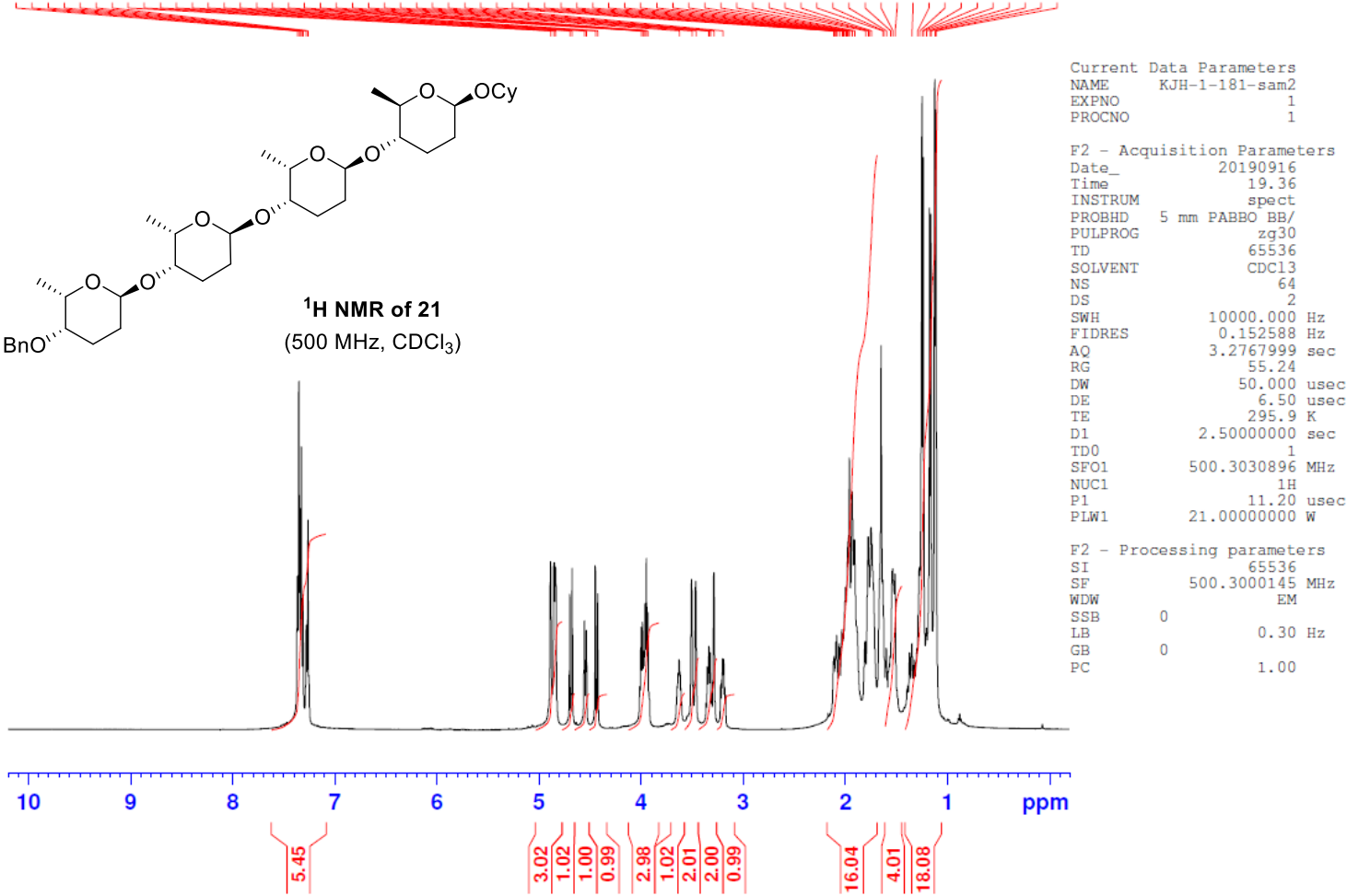

KJH-1-181-sam2 21320190916

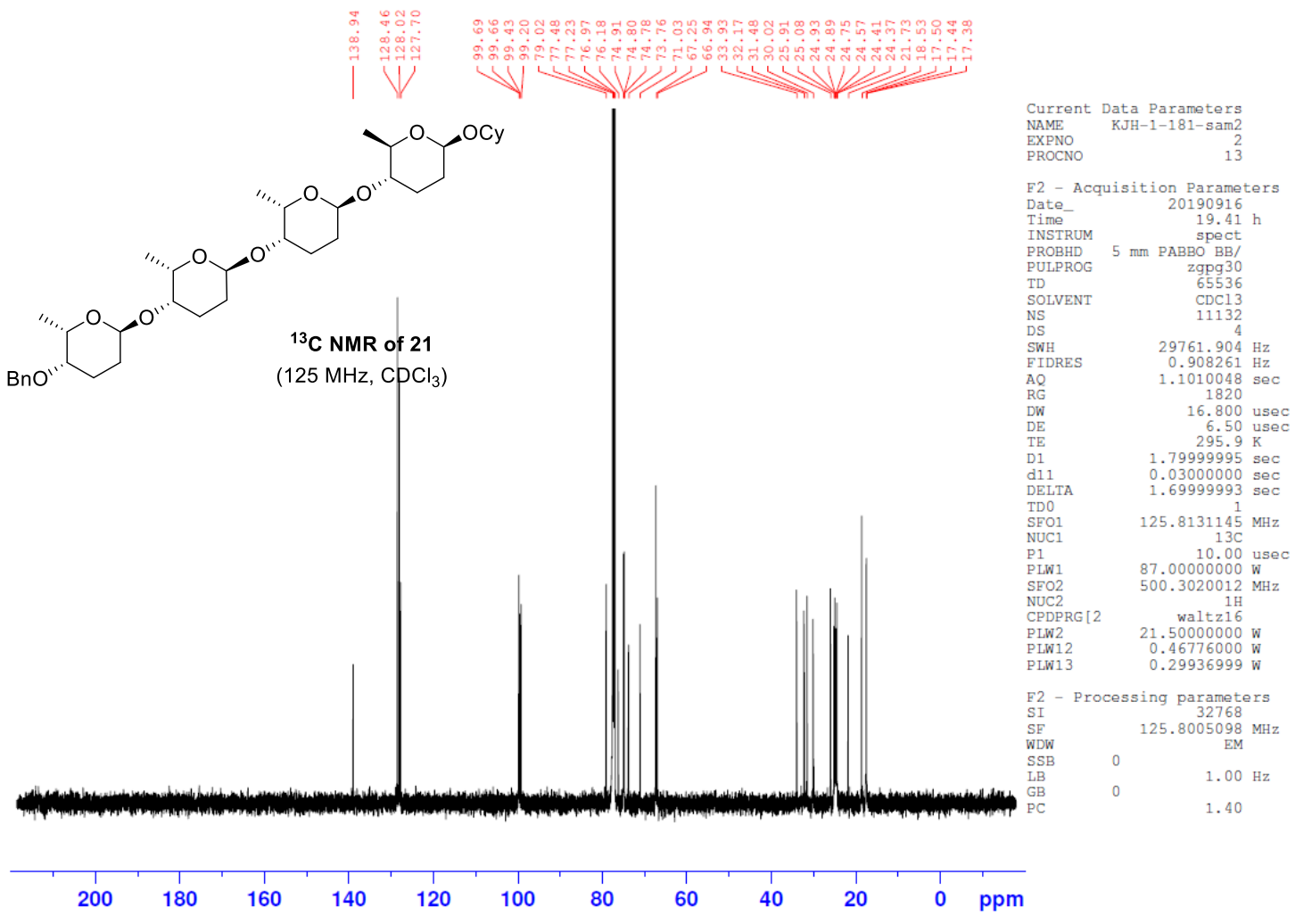




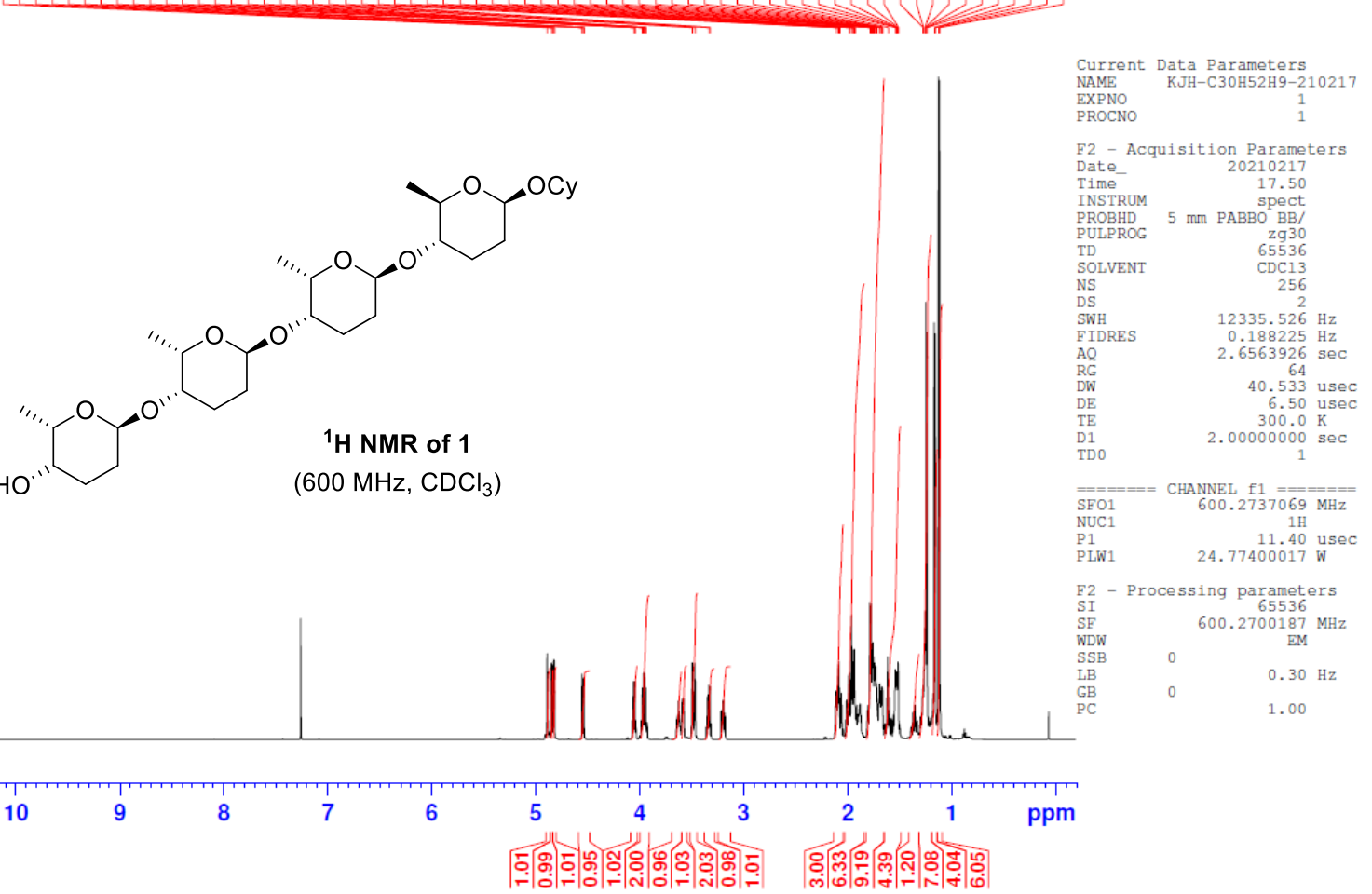

KJH-C30H52H9-210217

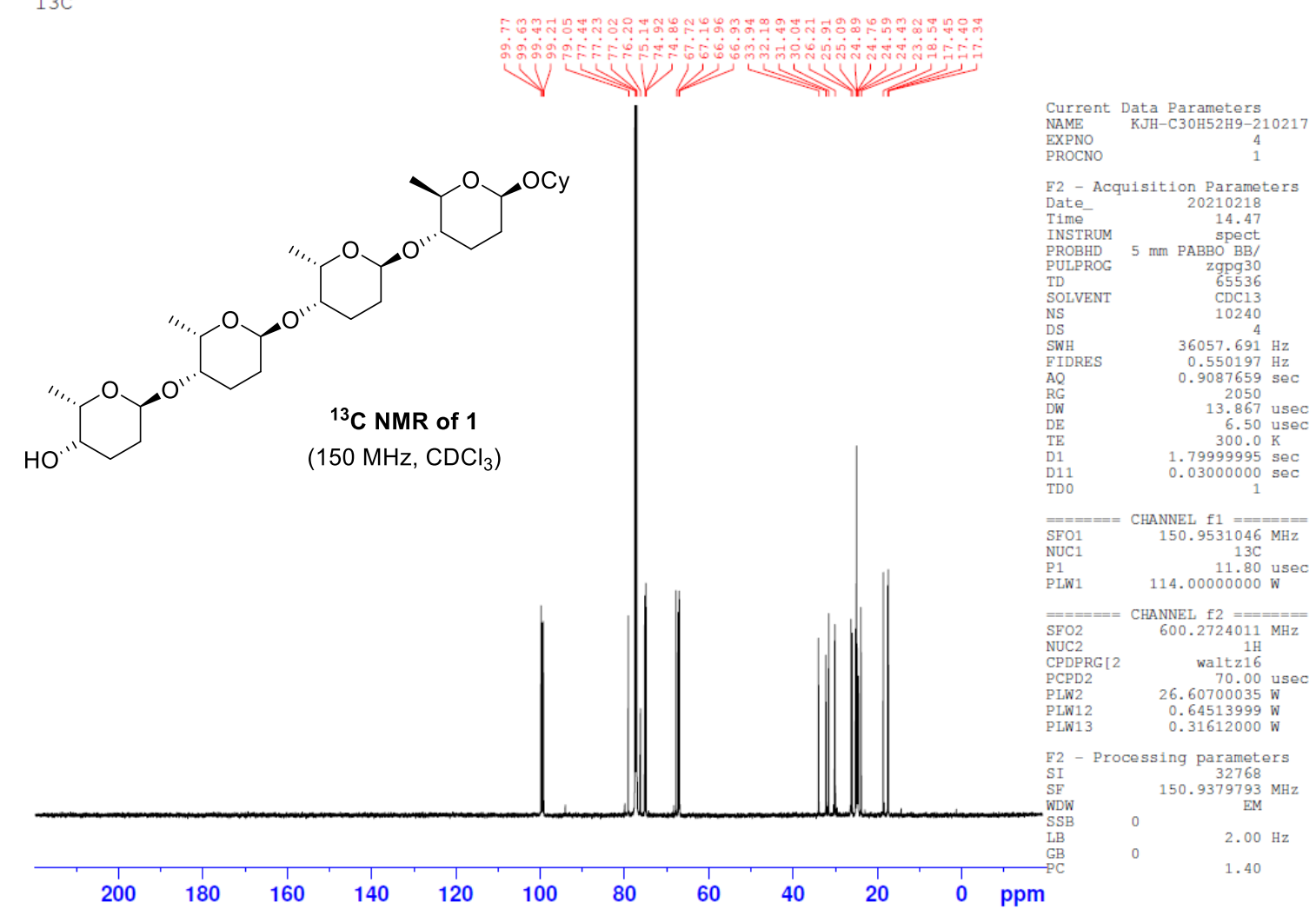


COSY of $\mathbf{1}$

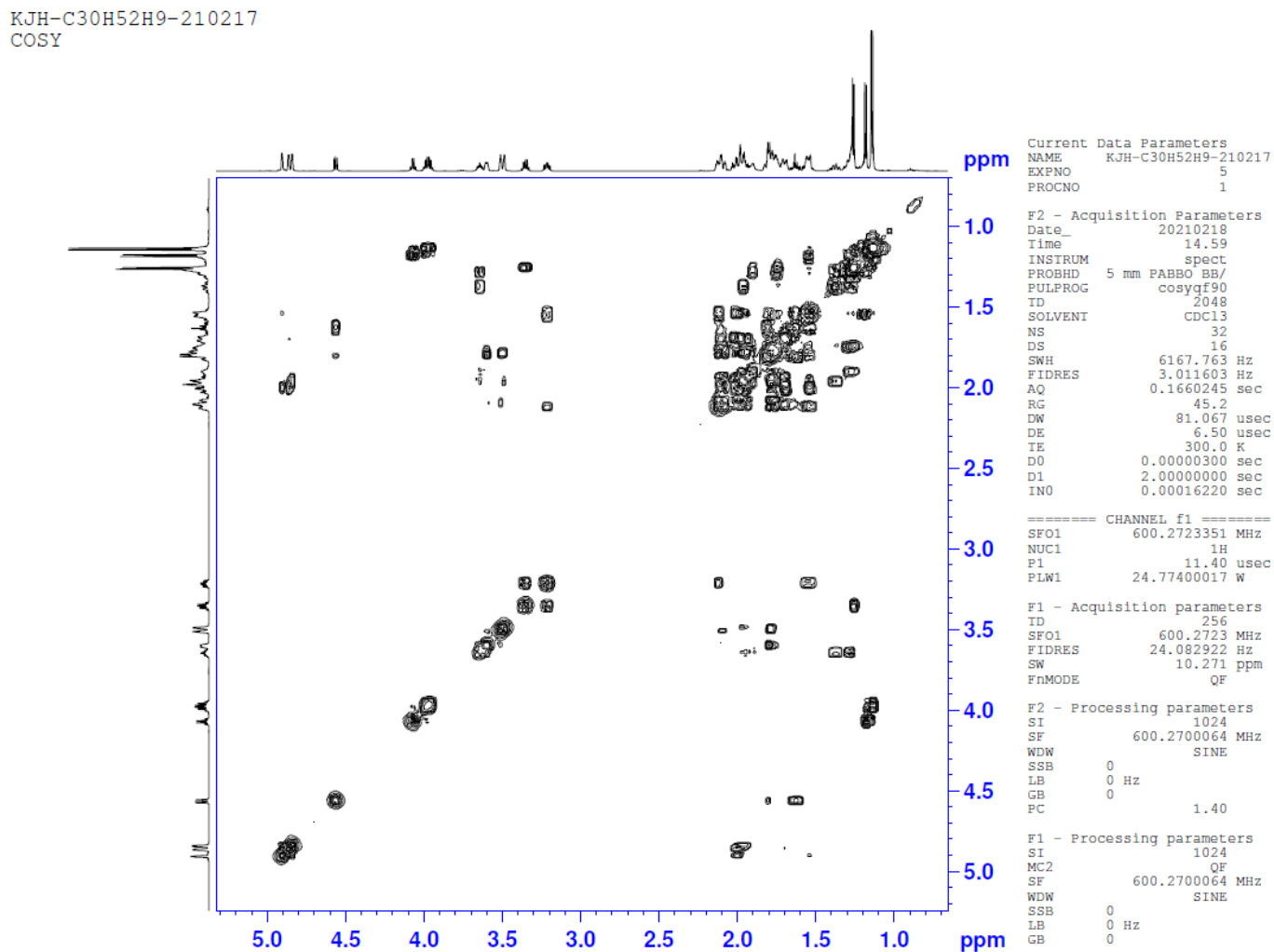

ROESY of 1

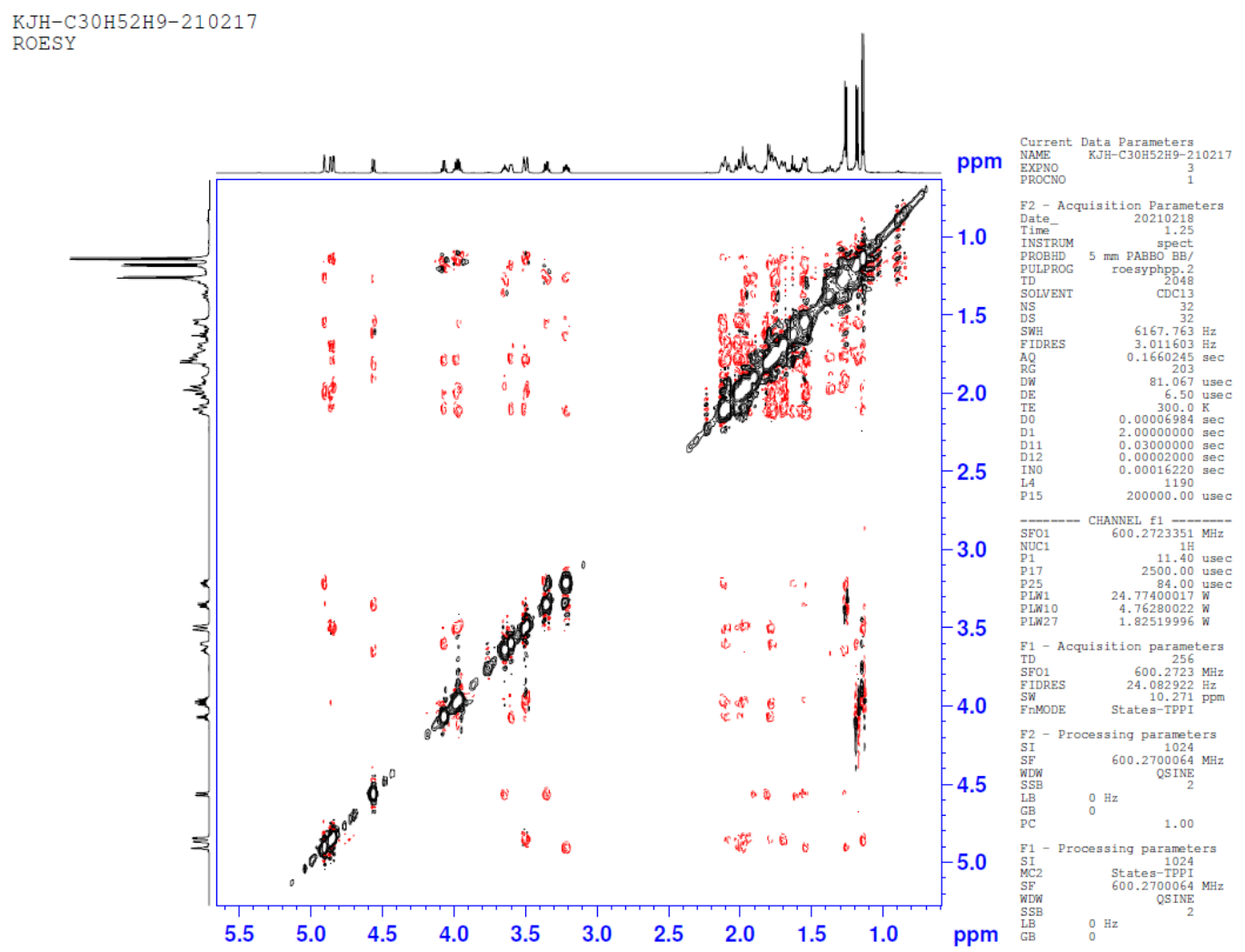


HSQC of $\mathbf{1}$

$\mathrm{KJH}-\mathrm{C} 30 \mathrm{H} 52 \mathrm{H} 9-210217$

$\mathrm{HSQC}$

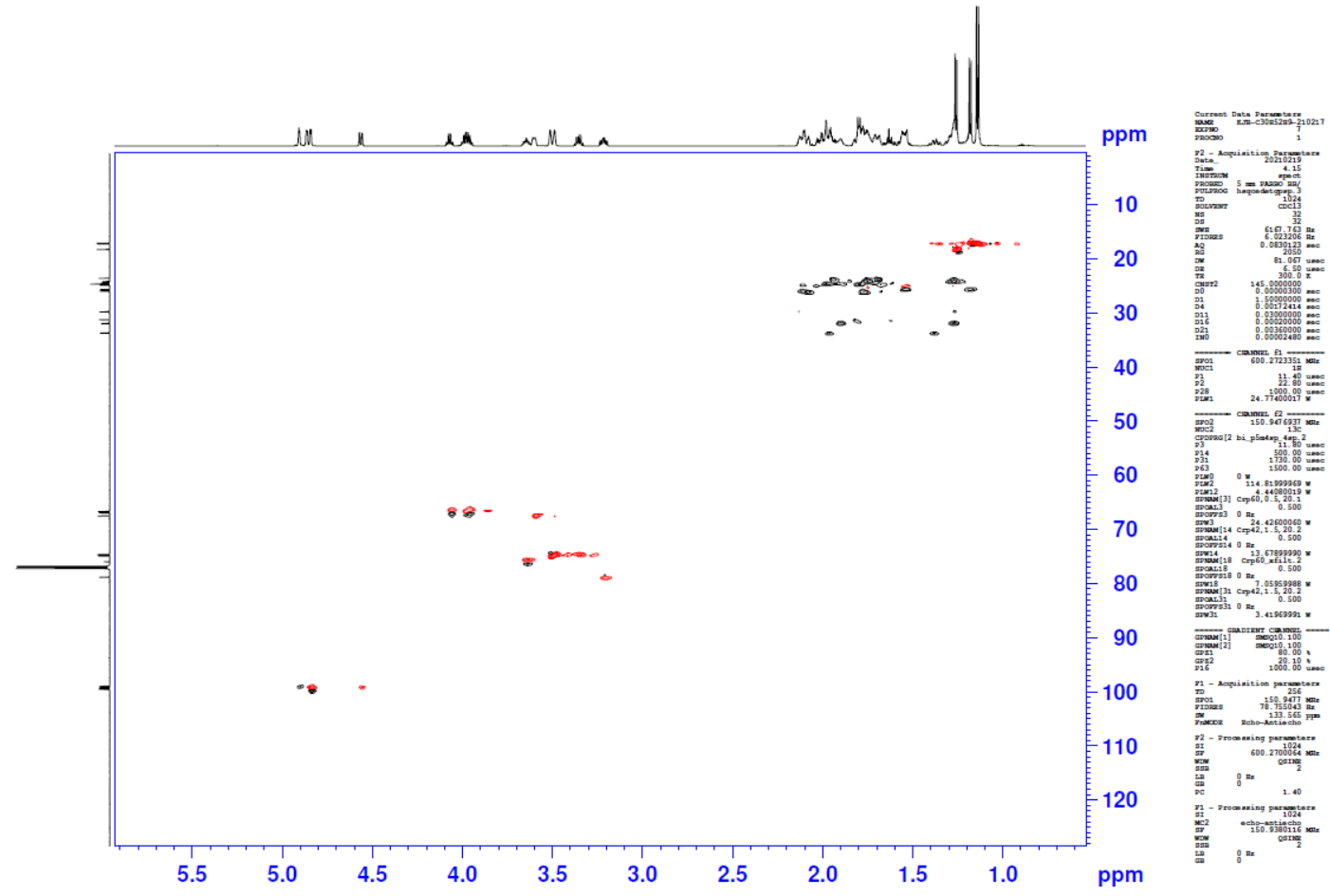

HMBC of 1

KJH-C30H52H9-210217

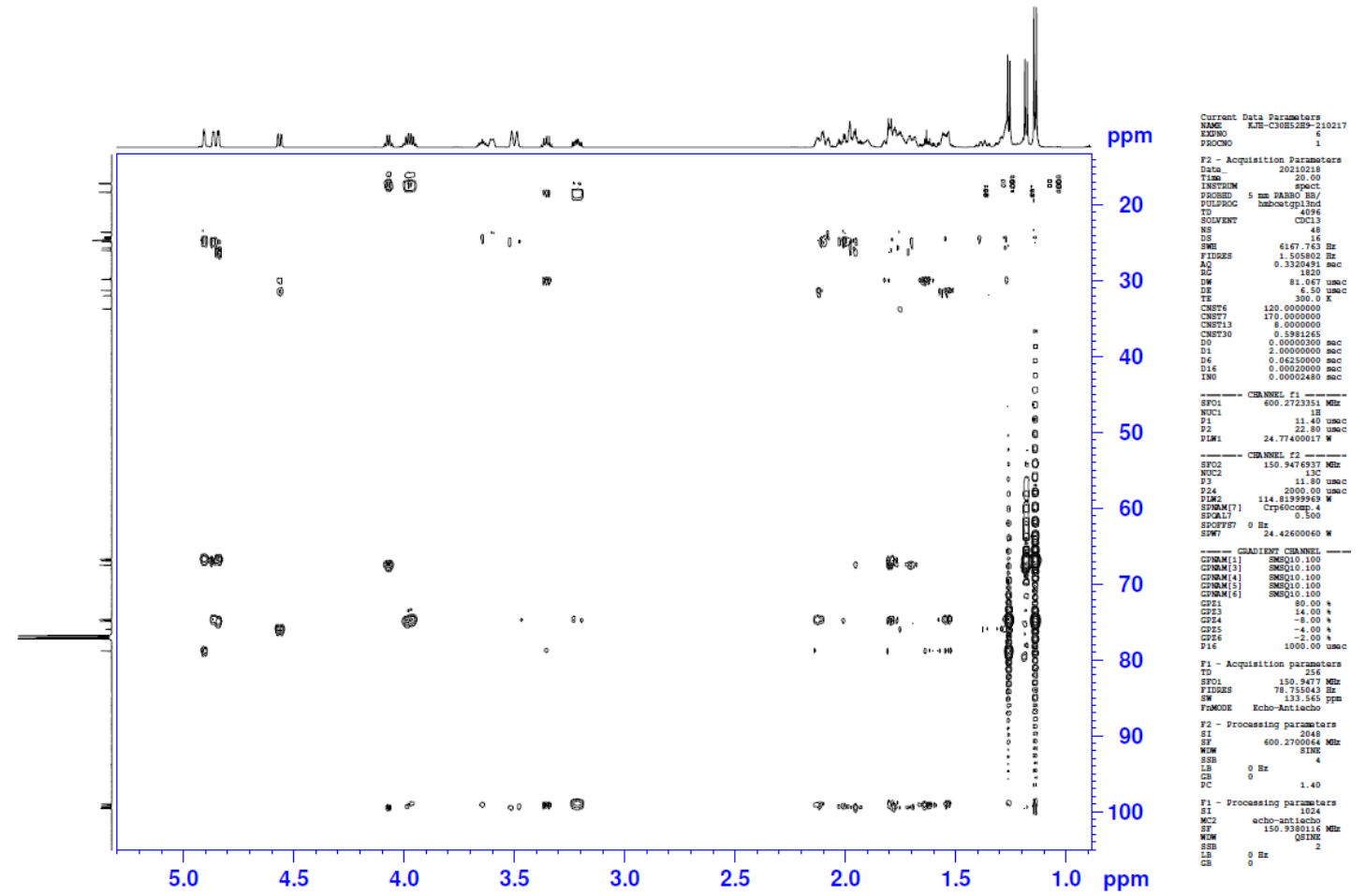


10. ${ }^{1} \mathrm{H}$ NMR of Crude mixture: Determination of $\mathbf{d} . \mathbf{r}$ for the addition reaction

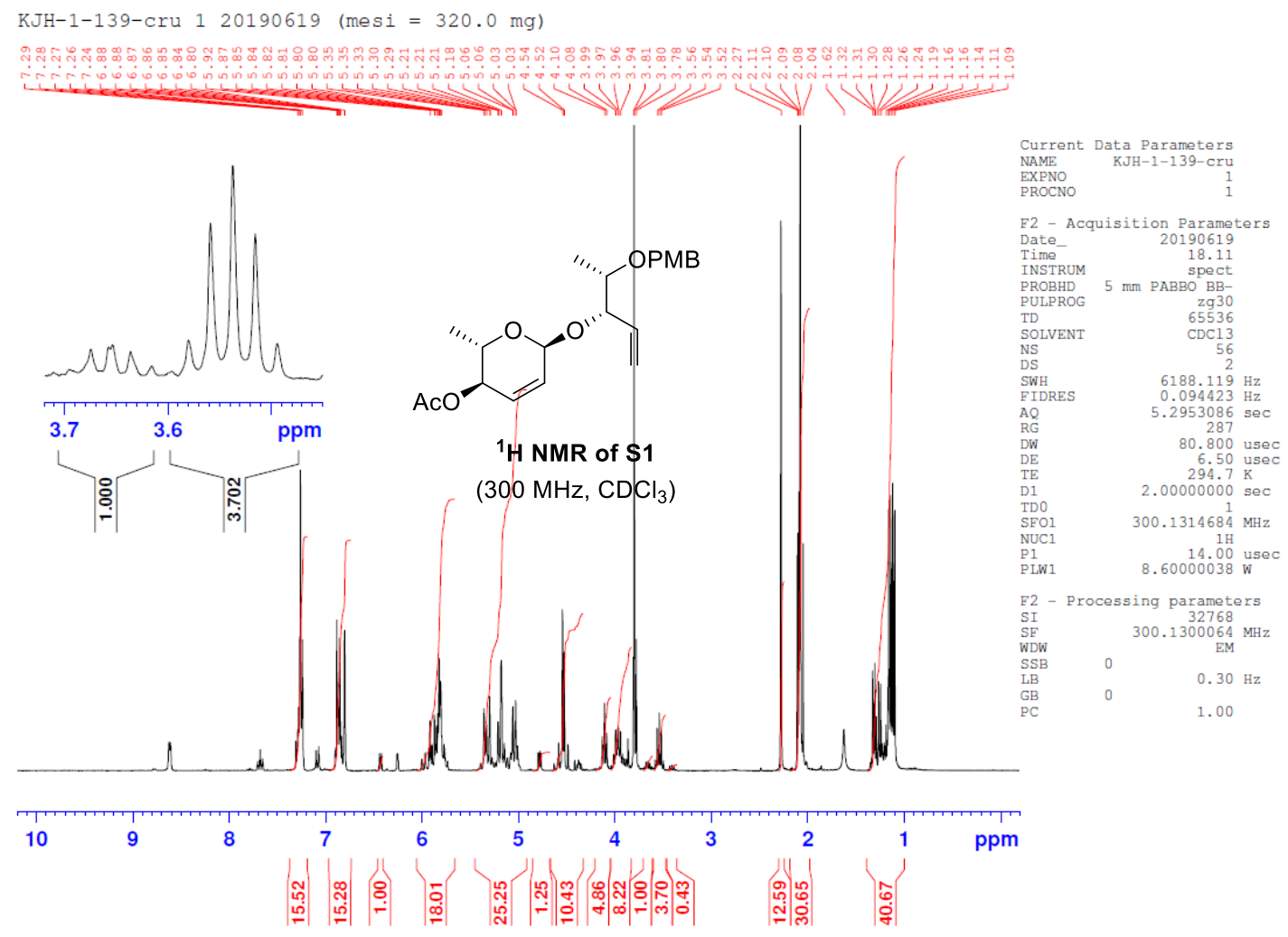

KJH-1-131-Cru 1120190526 (mesi = 7.5 mg)

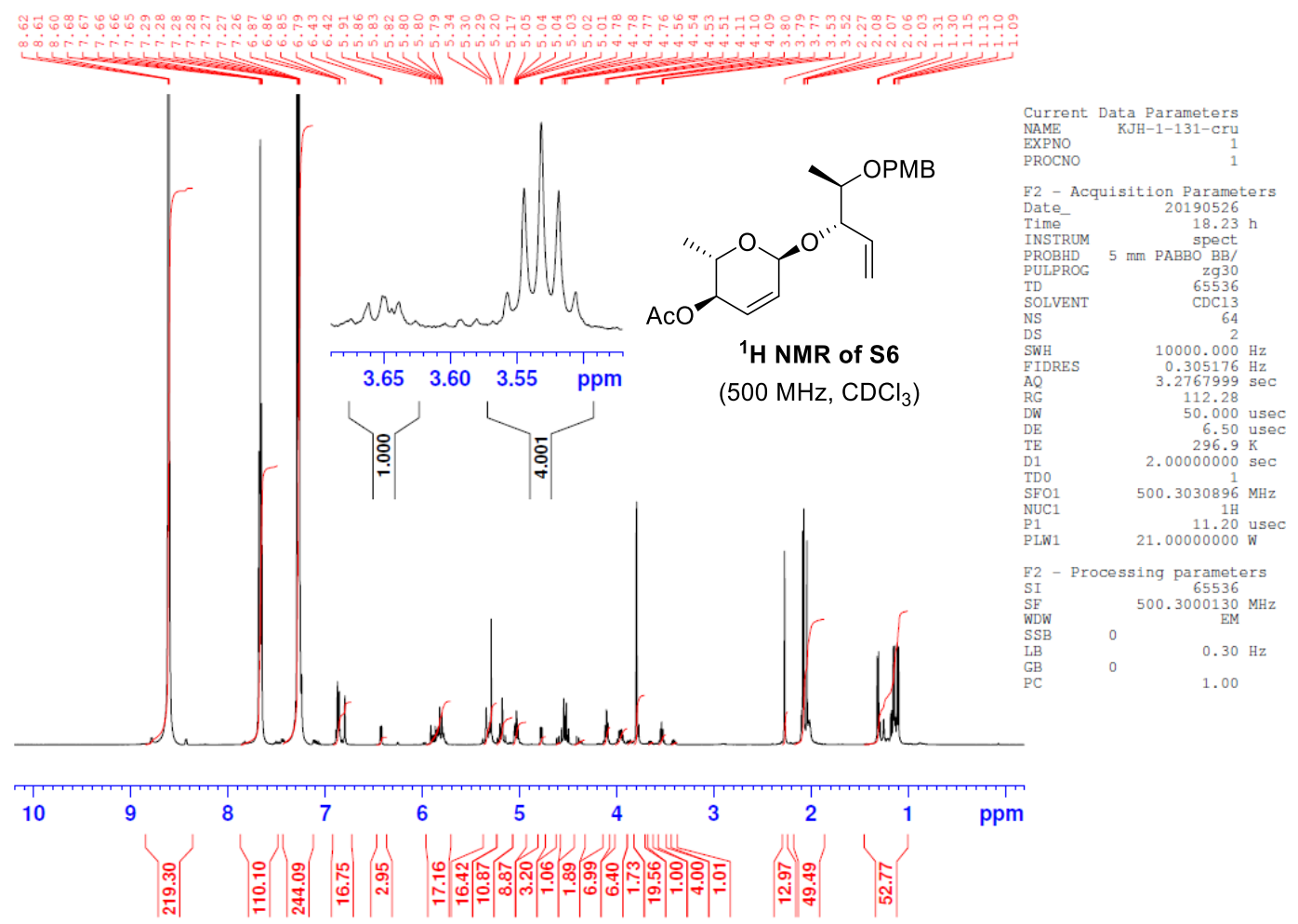




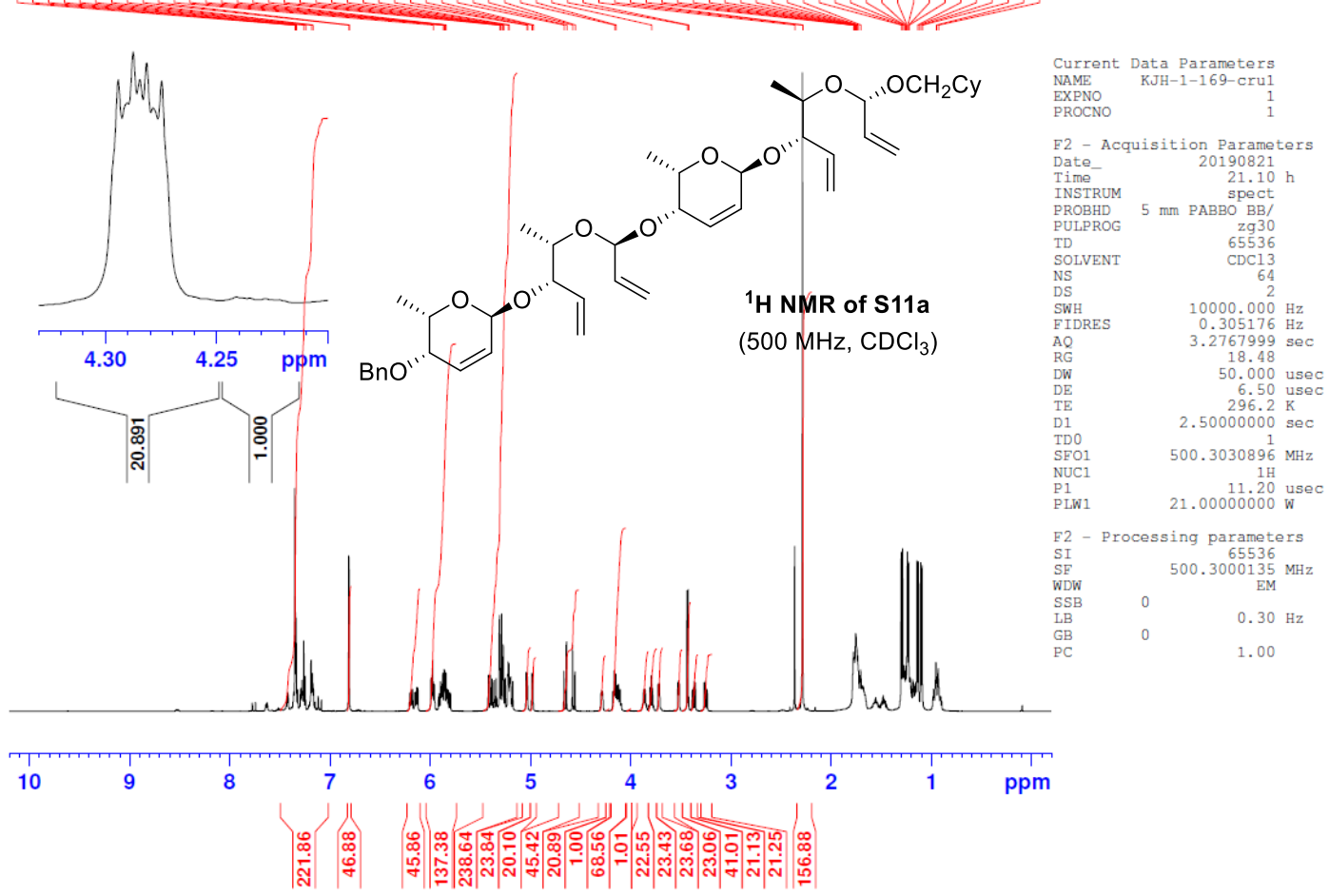

KJH-1-168-crul 1120190821 (mesi $=7.4 \mathrm{mg}$ )

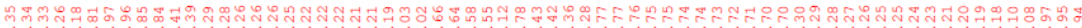
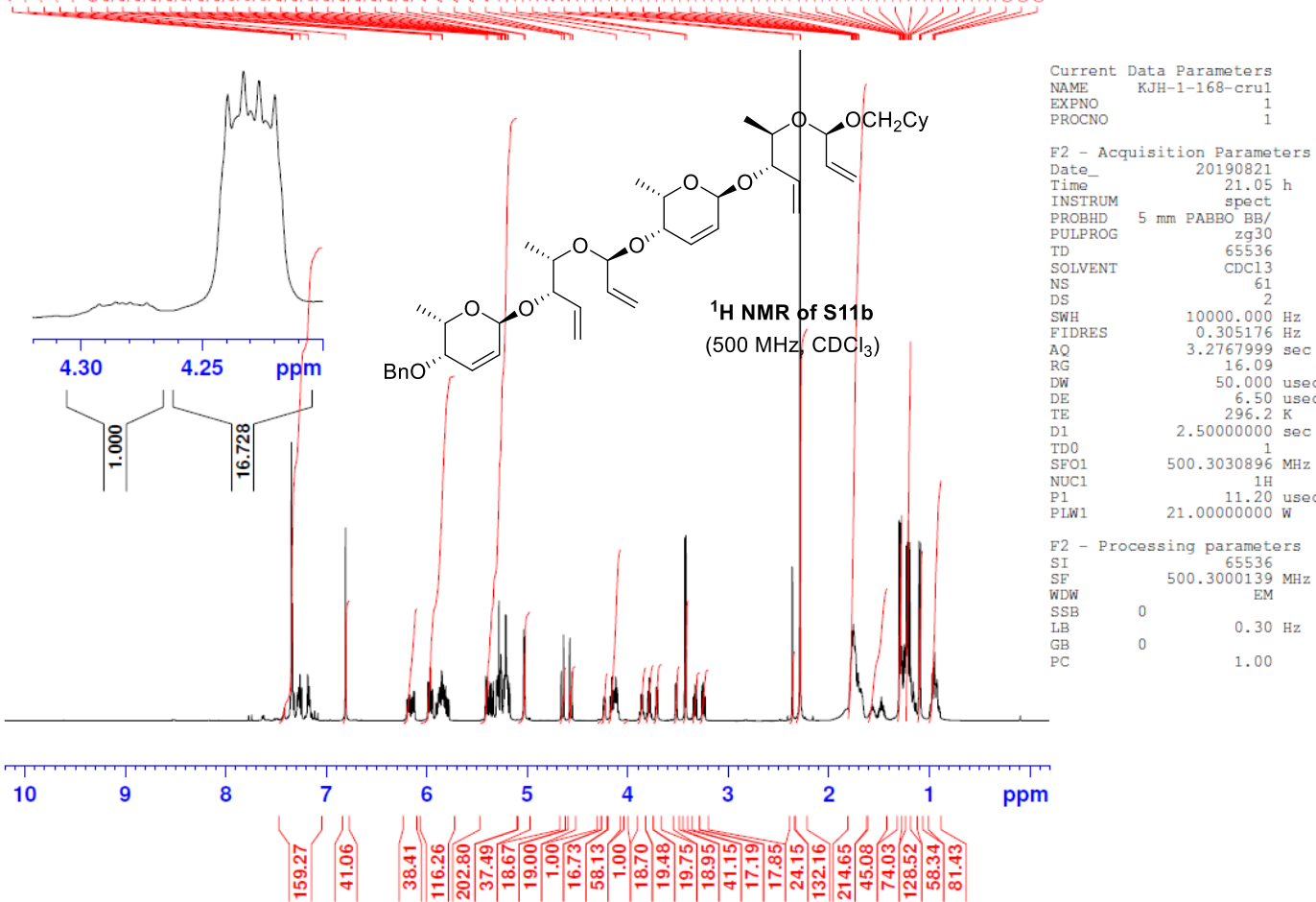


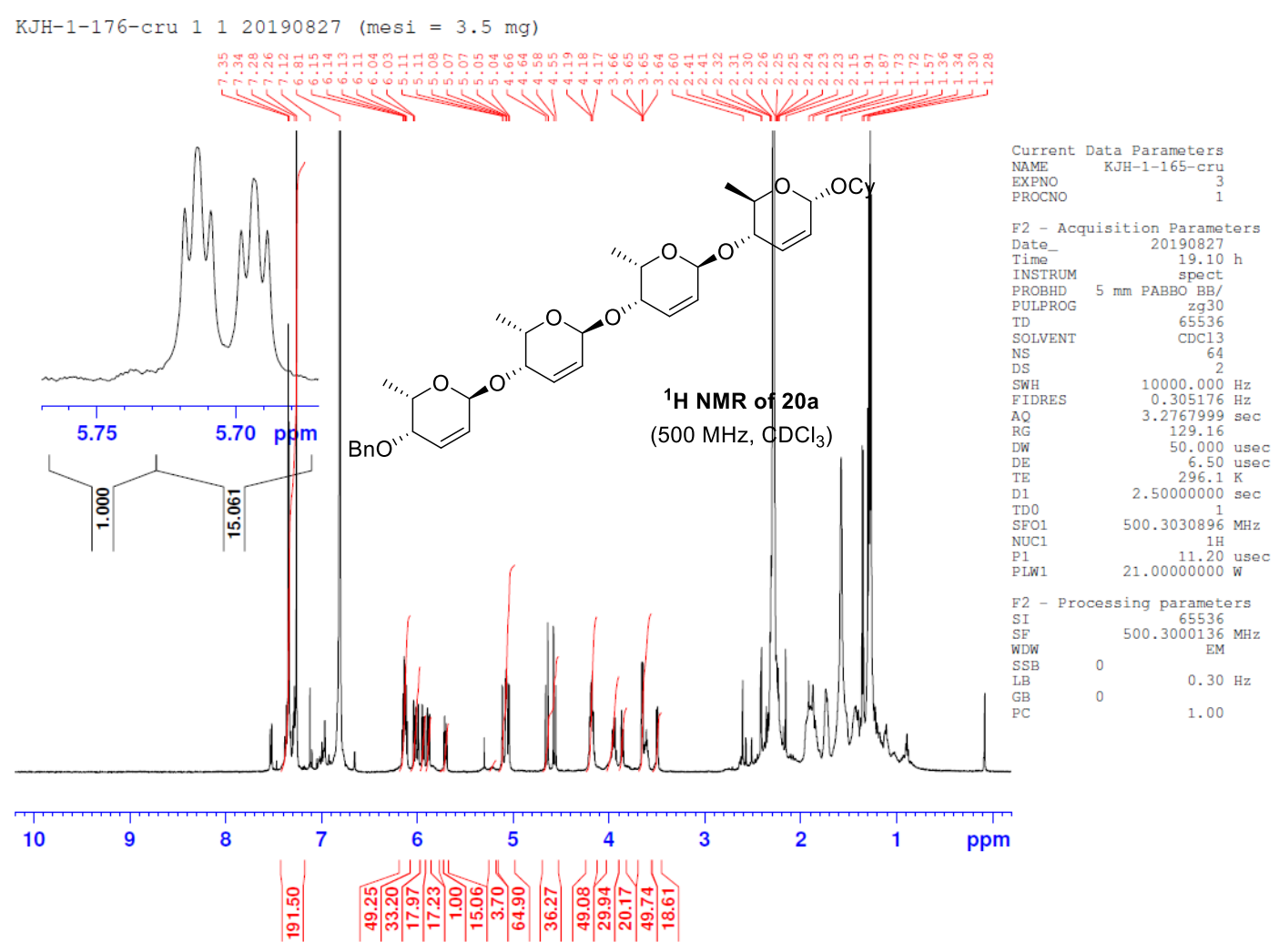

KJH-1-179-P-090519

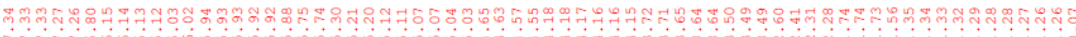
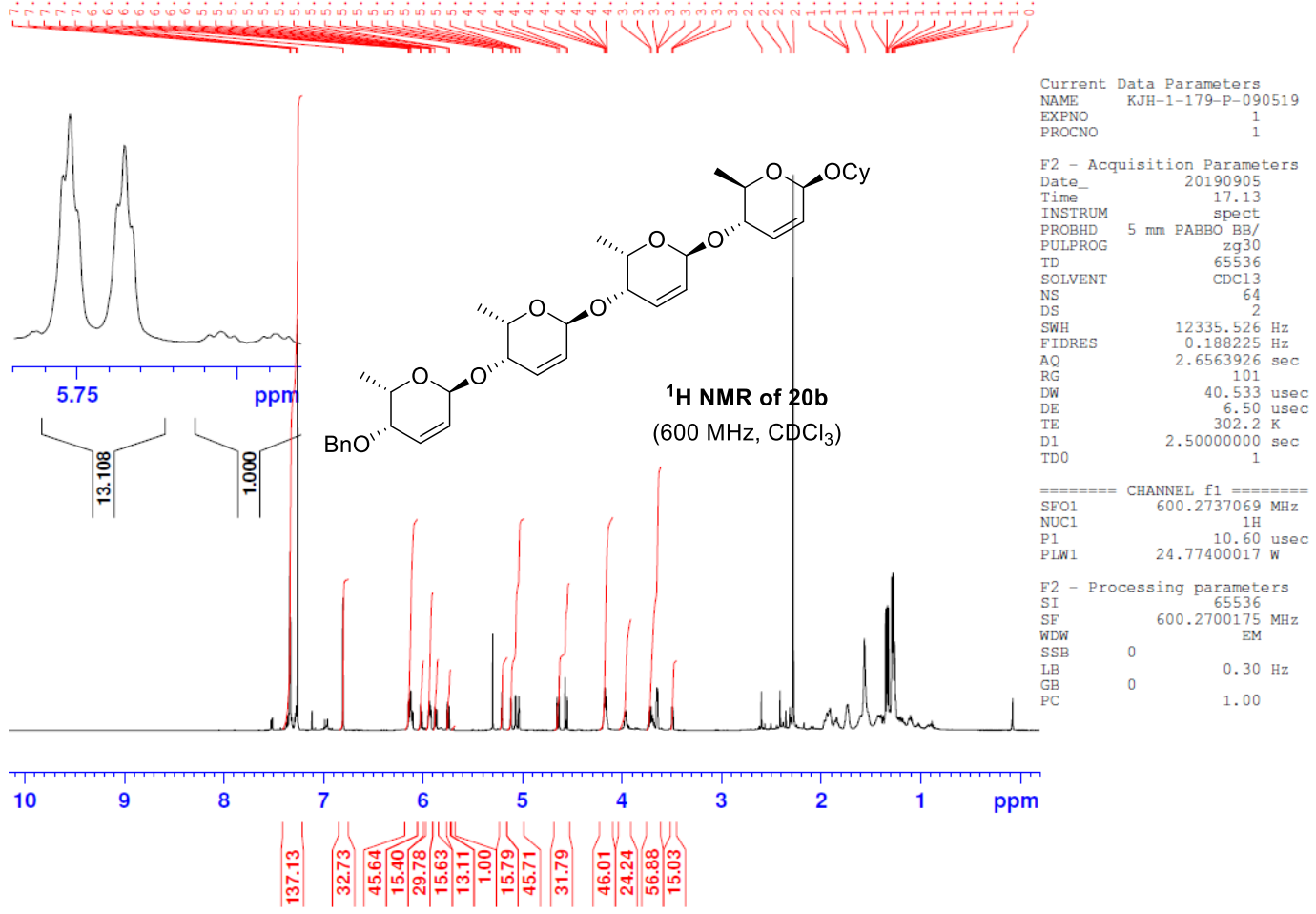